\title{
Low-Flow Characteristics at Selected Sites on Streams in Southern and Western Puerto Rico
}

By Luis Santiago-Rivera

U.S. GEOLOGICAL SURVEY

Water-Resources Investigations Report 95-4147

Prepared in cooperation with the

PUERTO RICO AQUEDUCT AND SEWER AUTHORITY and the PUERTO RICO ENVIRONMENTAL QUALITY BOARD

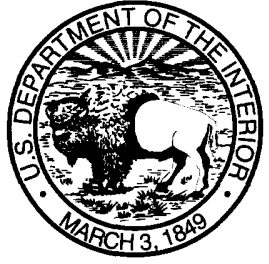




\title{
U.S. DEPARTMENT OF THE INTERIOR BRUCE BABBITT, Secretary
}

\author{
U.S. GEOLOGICAL SURVEY
}

Gordon P. Eaton, Director

For additional information write to:

District Chief

U.S. Geological Survey

GSA Center, Suite 400-15

651 Federal Drive

San Juan, Puerto Rico 00965
Copies of this report can be purchased from:

U.S. Geological Survey

Earth Science Information Center

Open-File Reports Section, MS 517

Box 25286, Denver Federal Center

Denver, CO 80225 


\section{CONTENTS}

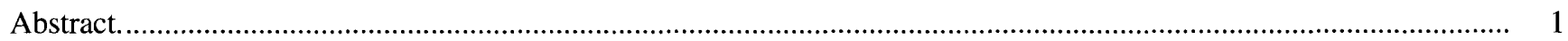

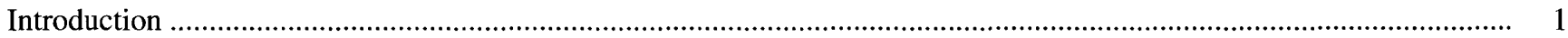

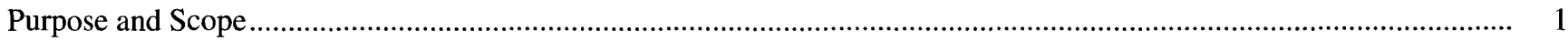

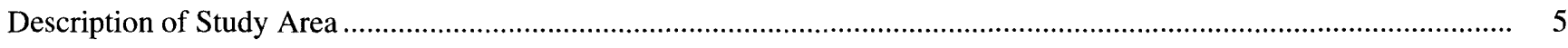

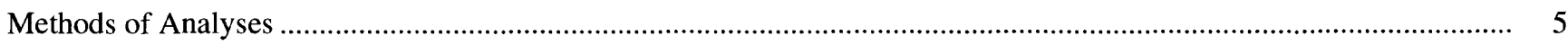

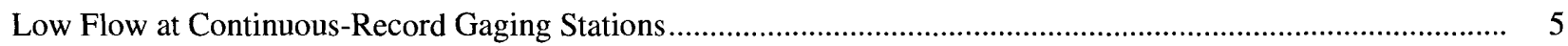

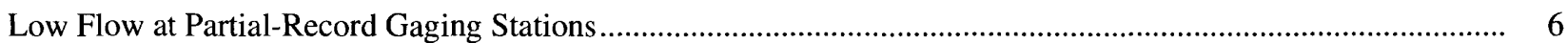

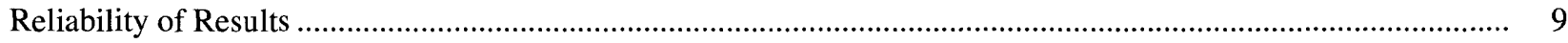

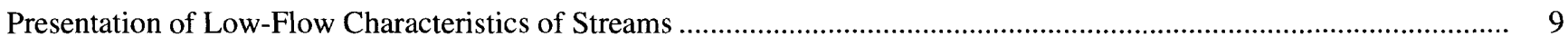

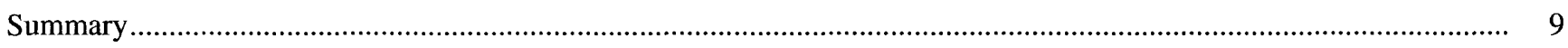

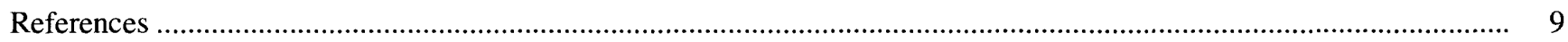

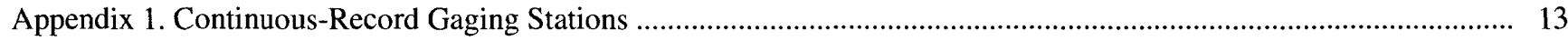

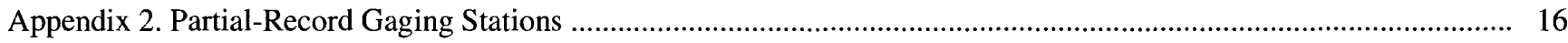

\section{FIGURES}

1-3. Map showing:

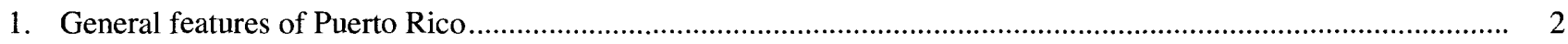

2. Location of low-flow continuous-record gaging stations in southern and western Puerto Rico ............................ 3

3. Location of low-flow partial-record gaging stations in southern and western Puerto Rico ................................

4. Computer generated low-flow frequency curve and graphical low-flow frequency curve for the Río Grande de Añasco station near San Sebastián, Puerto Rico

5. Graph showing relation between concurrent discharges at a partial-record station and a nearby continuous-record station 


\section{APPENDIX 1. CONTINUOUS-RECORD GAGING STATIONS}

\section{Río Grande de Patillas Basin}

50092000 Río Grande de Patillas near Patillas, Puerto Rico

\section{Río Inabón Basin}

50112500 Río Inabón at Real Abajo, Puerto Rico

\section{Río Bucaná Basin}

50114000 Río Cerrillos near Ponce, Puerto Rico

Río Portugués Basin

50115000 Río Portugués near Ponce, Puerto Rico

\section{Río Guayanilla Basin}

50124200 Río Guayanilla near Guayanilla, Puerto Rico

\section{Río Guanajibo Basin}

50136000 Río Rosario at Rosario, Puerto Rico

50138000 Río Guanajibo near Hormigueros, Puerto Rico

\section{Río Grande de Añasco Basin}

50141000 Río Blanco near Adjuntas, Puerto Rico

50144000 Río Grande de Añasco near San Sebastián, Puerto Rico

\section{APPENDIX 2. PARTIAL-RECORD GAGING STATIONS}

\section{Río Seco Basin}

50097000 Quebrada Cimarrona near Jobos, Puerto Rico

50097800 Río Seco near Central Guamaní, Puerto Rico

\section{Río Salinas Basin}

50100200 Río Lapa near Rabo del Buey, Puerto Rico.

50100300 Río Jájome at Jájome, Puerto Rico

50100450 Río Majada at La Plena, Puerto Rico

\section{Río Jueyes Basin}

50103000 Río Jueyes near Jauca, Puerto Rico

\section{Río Coamo Basin}

50104000 Río Coamo near Pasto, Puerto Rico.

50105400 Río Cuyón at La Guava, Puerto Rico

50105600 Río Cuyón near Coamo, Puerto Rico

50105900 Quebrada Montería near Coamo, Puerto Rico

50106100 Río Coamo at Coamo, Puerto Rico

50106600 Río de La Mina near Coamo, Puerto Rico

50106650 Río del Pasto near Coamo, Puerto Rico

50106700 Río de La Mina at Coamo, Puerto Rico

50106820 Río Coamo at Baños de Coamo, Puerto Rico. 


\section{APPENDIX 2. PARTIAL-RECORD GAGING STATIONS-Continued}

\section{Río Descalabrado Basin}

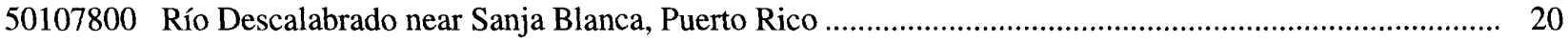

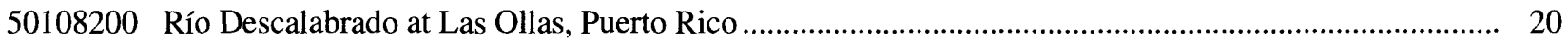

\section{Río Cañas Basin}

50109000 Río Cañas near Juana Díaz, Puerto Rico.

50109500 Río Cañas near Santa Isabel, Puerto Rico.

\section{Río Jacaguas Basin}

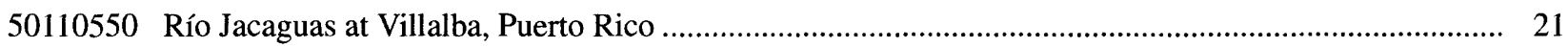

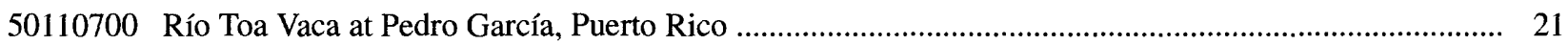

50110900 Río Toa Vaca upstream from Lago Toa Vaca, Puerto Rico ...................................................... 21

50111720 Quebrada Guanábana near Juana Díaz, Puerto Rico ........................................................... 21

\section{Río Inabón Basin}

50112400 Río Inabón at Real Anón, Puerto Rico.

50112700 Río Guayo near Collores, Puerto Rico.

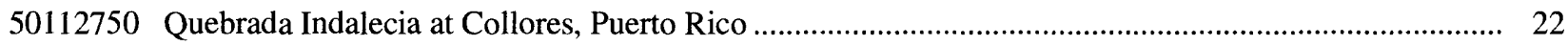

50112800 Río Guayo upstream from Diversion at Collores, Puerto Rico................................................... 22

\section{Río Bucaná Basin}

50113790 Río San Patricio upstream from Lago Cerrillos, Puerto Rico ................................................... 23

50113800 Río Cerrillos upstream from Lago Cerrillos, Puerto Rico ........................................................... 23

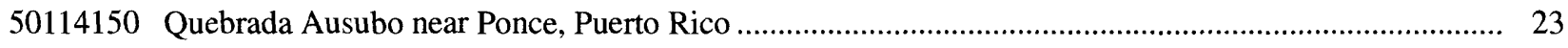

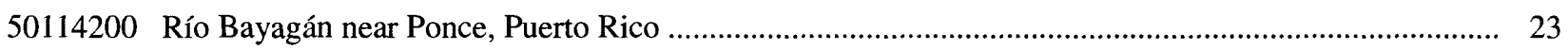

\section{Río Portugués Basin}

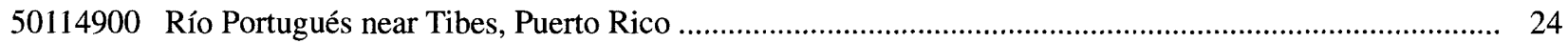

50115400 Río Portugués near Ponce, Puerto Rico .............................................................................. 24

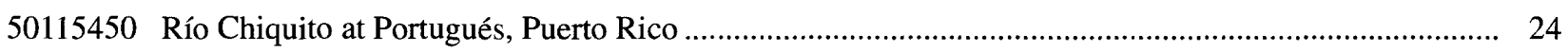

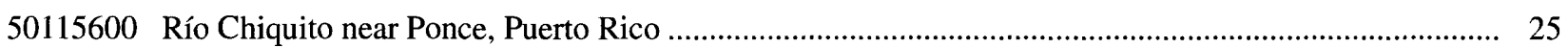

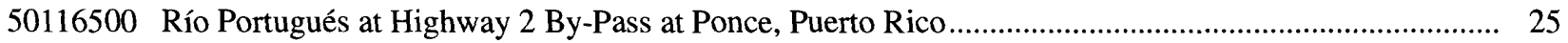

\section{Río Matilde Basin}

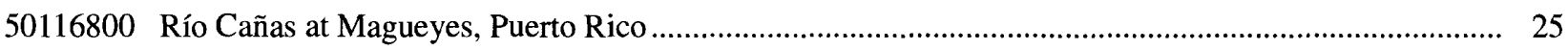

50116970 Río Cañas downstream from Las Américas Ave., Puerto Rico .................................................... 26

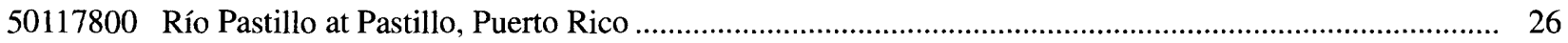

50118300 Río Pastillo near Ponce, Puerto Rico .................................................................................. 26

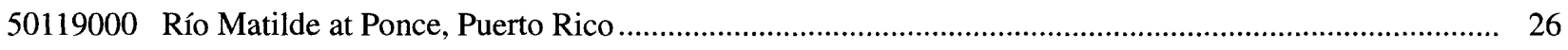

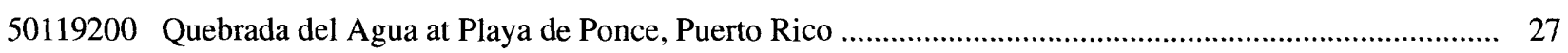

\section{Río Tallaboa Basin}

50120550 Río Tallaboa near Quebrada Ceiba, Puerto Rico ............................................................ 27

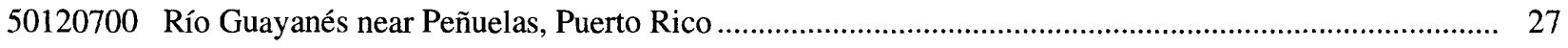


50121000 Río Tallaboa at Peñuelas, Puerto Rico

50122000 Río Tallaboa at Tallaboa, Puerto Rico

\section{Río Macaná Basin}

50122500 Río Macaná near Peñuelas, Puerto Rico

50122900 Río Macaná at Magas Arriba, Puerto Rico

\section{Río Guayanilla Basin}

50123100 Río Guayanilla at Pasto, Puerto Rico

50124600 Río Guayanilla near Central Rufina, Puerto Rico

\section{Río Yauco Basin}

50125000 Río Yauco near Lago Lucchetti Damsite, Puerto Rico

50125500 Río Naranjo near Lago Lucchetti Damsite, Puerto Rico

50125600 Quebrada Grande near Lago Lucchetti Damsite, Puerto Rico 30

50125860 Río Duey at Duey, Puerto Rico

\section{Río Loco Basin}

50128450 Quebrada Grande upstream from Lago Loco, Puerto Rico.

50128500 Río Loco upstream from Lago Loco, Puerto Rico

50129200 Quebrada Susúa at Palomas, Puerto Rico

\section{Río Guanajibo Basin}

50130400 Río Grande near Sabana Grande, Puerto Rico

50130500 Río Guanajibo at La Pica, Puerto Rico

50130800 Río Flores near Sabana Grande, Puerto Rico

50131010 Río Cruces near Sabana Grande, Puerto Rico.

50131800 Río Cupeyes near San Germán, Puerto Rico

50132010 Río Guanajibo below San Germán, Puerto Rico

50133000 Río Caín near San Germán, Puerto Rico

50133800 Río Duey near Rosario, Puerto Rico

50134600 Río Hoconuco near San Germán, Puerto Rico.

50135000 Río Hoconuco (Duey) near San Germán, Puerto Rico

50135700 Río Maricao at Maricao, Puerto Rico

50135800 Río Rosario at Las Vegas, Puerto Rico

50136400 Río Rosario near Hormigueros, Puerto Rico

50136500 Río Rosario near Hormigueros, Puerto Rico

50137800 Río Viejo near Cabo Rojo, Puerto Rico

\section{Quebrada Maga Basin}

50138100 Quebrada Maga near Guanajibo, Puerto Rico

\section{Río Hondo Basin}

50138200 Río Hondo near Guanajibo, Puerto Rico 


\section{APPENDIX 2. PARTIAL-RECORD GAGING STATIONS-Continued}

\section{Quebrada Sábalos Basin}

50138300 Quebrada Sábalos near Mayagüez, Puerto Rico

\section{Río Yagüez Basin}

50138900 Río Yagüez at Balboa, Puerto Rico

\section{Río Grande de Añasco Basin}

50140300 Río Guilarte near Adjuntas, Puerto Rico ..................................................................................... 38

50140800 Río Limani near Adjuntas, Puerto Rico ......................................................................................... 38

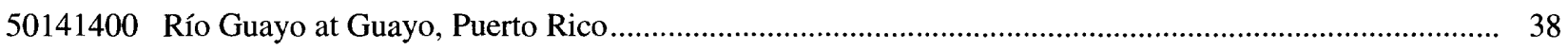

50142000 Río Blanco at La Torre, Puerto Rico …................................................................................... 39

50142100 Quebrada de Los Plátanos at Marisol, Puerto Rico ......................................................................... 39

50142300 Río Prieto at Indiera Alta, Puerto Rico ................................................................................................... 39

50142710 Río Prieto at Río Prieto, Puerto Rico …........................................................................................... 40

50142900 Río Prieto at Pezuela, Puerto Rico .................................................................................................... 40

50143000 Río Grande de Añasco near Lares, Puerto Rico................................................................................. 40

50143104 Río Lajas near Maricao, Puerto Rico ............................................................................................... 41

50143108 Río Guaba near Maricao, Puerto Rico ................................................................................................... 41

50143150 Río Bucarabones near Las Marías, Puerto Rico ............................................................................... 41

50143200 Río Guaba near Las Marías, Puerto Rico .................................................................................... 41

50143400 Quebrada Las Cañas at Perchas, Puerto Rico ................................................................................ 42

50143500 Río Mayagüecilla at Las Marías, Puerto Rico ………................................................................. 42

50143800 Río Grande de Añasco near Las Marías, Puerto Rico............................................................................. 42

50143900 Río Arenas at Las Marías, Puerto Rico ….......................................................................................... 42

50144200 Quebrada Cerro Gordo near Cerro Gordo, Puerto Rico.................................................................. 43

50144900 Río Humata near E1 Espino, Puerto Rico .................................................................................. 43

50145000 Río Grande de Añasco at El Espino, Puerto Rico.......................................................................... 43

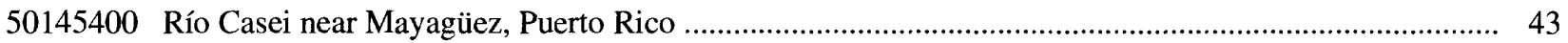

50146000 Río Grande de Añasco at Añasco Arriba, Puerto Rico ..................................................................... 44

50146002 Río Cañas at Río Cañas Arriba, Puerto Rico ...................................................................................... 44

50146005 Río Cañas at Río Cañas Abajo, Puerto Rico .................................................................................... 44

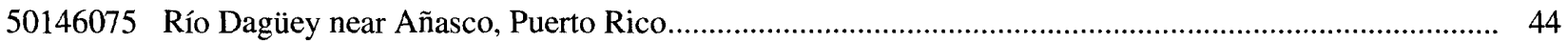

\section{Rio Grande Basin}

50146200 Río Grande near Rincón, Puerto Rico................................................................................... 45

\section{Río Ingenio Basin}

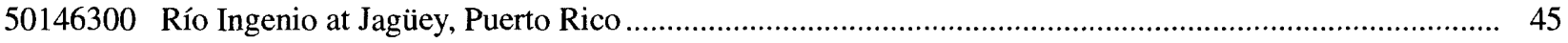

50146400 Río Ingenio near Aguada, Puerto Rico …................................................................................... 45

Río Culebra Basin

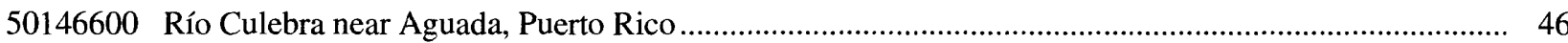


CONVERSION FACTORS AND ACRONYMS

\begin{tabular}{rll}
\hline Multiply & By & To obtain \\
\hline inch (in.) & 25.4 & millimeter \\
foot (ft) & 0.3048 & meter \\
mile (mi) & 1.609 & kilometer \\
acre & 4,047 & square meter \\
square mile $\left(\mathrm{mi}^{2}\right)$ & 2.590 & square kilometer \\
foot per mile $(\mathrm{ft} / \mathrm{mi})$ & 0.1894 & meter per kilometer \\
cubic foot per second $\left(\mathrm{ft}^{3} / \mathrm{s}\right)$ & 0.02832 & cubic meter per second \\
\hline
\end{tabular}

Acronyms used in this report:

PRASA Puerto Rico Aqueduct and Sewer Authority

PREQB Puerto Rico Environmental Quality Board

USGS United States Geological Survey 


\title{
Low-Flow Characteristics at Selected Sites on Streams in Southern and Western Puerto Rico
}

\author{
By Luis Santiago-Rivera
}

\section{ABSTRACT}

Knowledge of the magnitude and frequency of low flows is important for the optimal development of surface-water resources in Puerto Rico. This report presents analyses of low-flow data for 9 continuous-record gaging stations and 105 partial-record stations in southern and western Puerto Rico. The report includes analyses of lowflow data and tabulations of computed low-flow magnitude and frequency characteristics for 7-, 14-, $30-, 60-$, and 90-consecutive days with recurrence intervals of 2 and 10 years for continuous-record gaging stations based on the log-Pearson Type III frequency distribution or graphically adjusted logPearson frequency curves. Estimates of low-flow characteristics are provided for partial-record stations for 7-, 14-, and 30-consecutive days with recurrence intervals of 2 and 10 years. Low-flow characteristics at partial-record stations were estimated based on the relation of base-flow discharge measurements at the partial-record stations and concurrent discharges at nearby continuous-record stations.

\section{INTRODUCTION}

A thorough analysis of low-flow magnitude and frequency characteristics of selected streams in Puerto Rico is necessary because of the increasing demand upon surface-water resources by industrial and public users, and the legal requirements for pollution control. As water demand and waste discharges to streams increase, knowledge of low-flow characteristics of streams becomes increasingly important. Low-flow information can be used as an index for water-

management regulations, to assess the water-supply potential, and to adequately evaluate the capacity of the stream to receive waste loads. A good understanding of minimum streamflow characteristics is also vital to preserve aquatic and wildlife habitats.

Low-flow discharge data for streams in Puerto Rico have been collected over a number of years. Lowflow characteristics have been published in reports by Cobb (1978), Colón-Dieppa and Quiñones-Aponte (1985), and Santiago-Rivera (1992). Since these studies were completed, additional streamflow data have been collected at continuous-record stations.

\section{PURPOSE AND SCOPE}

The purpose of this report is to present estimates of low-flow magnitude and frequency at selected sites on streams in southern and western Puerto Rico (fig. 1). In response to increasing needs for low-flow information, the U.S. Geological Survey (USGS), in cooperation with the Puerto Rico Aqueduct and Sewer Authority (PRASA) and the Puerto Rico Environmental Quality Board (PREQB) began a long-range study in 1983 to estimate low flows of streams in Puerto Rico.

The low-flow network in southern and western Puerto Rico includes 9 long-term continuous-record gaging stations (fig. 2) with at least 10 years of record, and 105 partial-record stations (fig. 3). These stations are fairly well distributed throughout southern and western Puerto Rico. Long-term continuous-record streamflow-gaging stations are sites where daily flow data are systematically collected over a period of 10 or more years. Gaging stations with less than 10 years of 


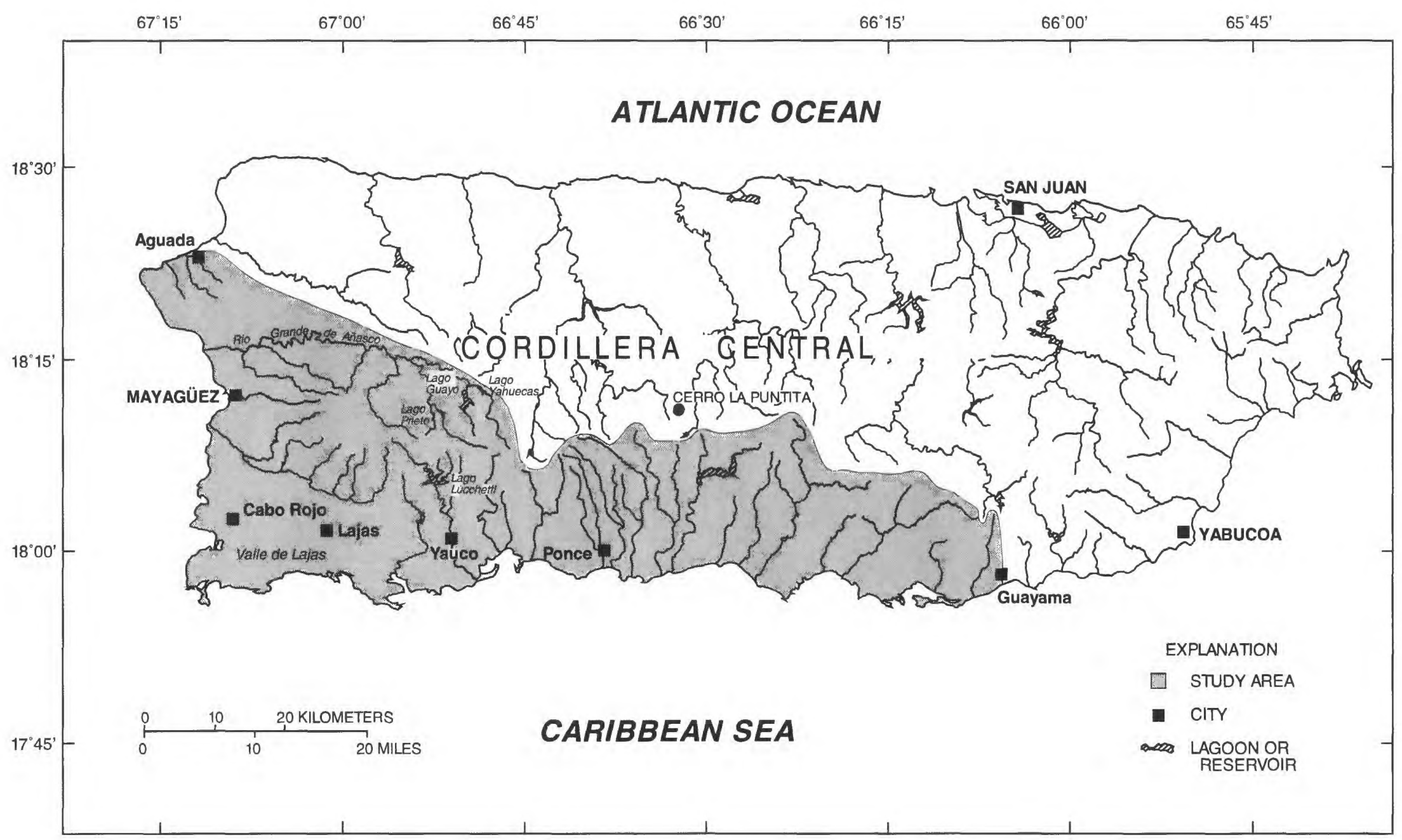

Figure 1. General features of Puerto Rico. 


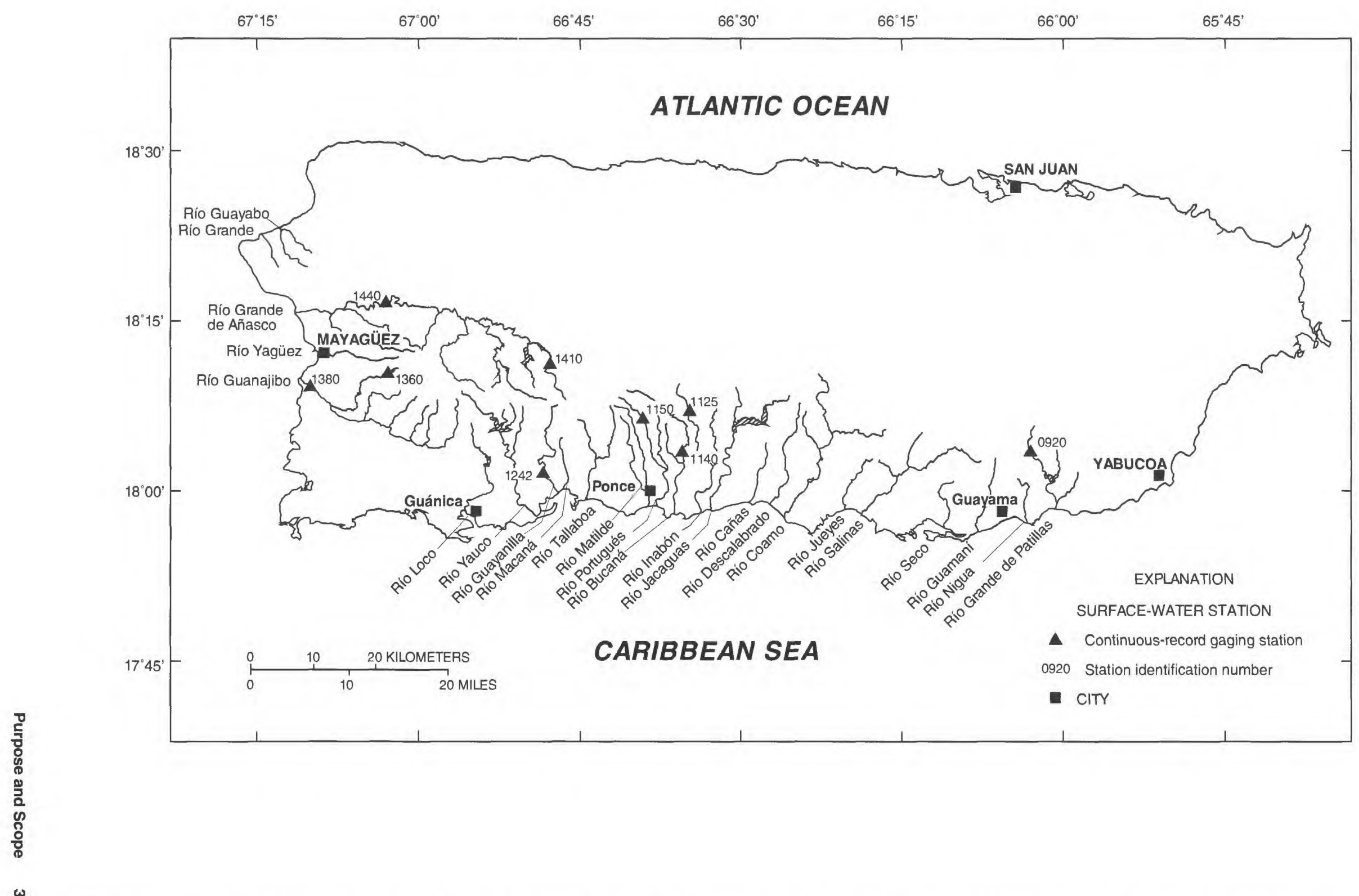

Figure 2. Location of low-flow continuous-record gaging stations in southern and western Puerto Rico. Site numbers on map refer to the third through sixth or eighth digit of the station number shown in the station headings of appendix 1. 


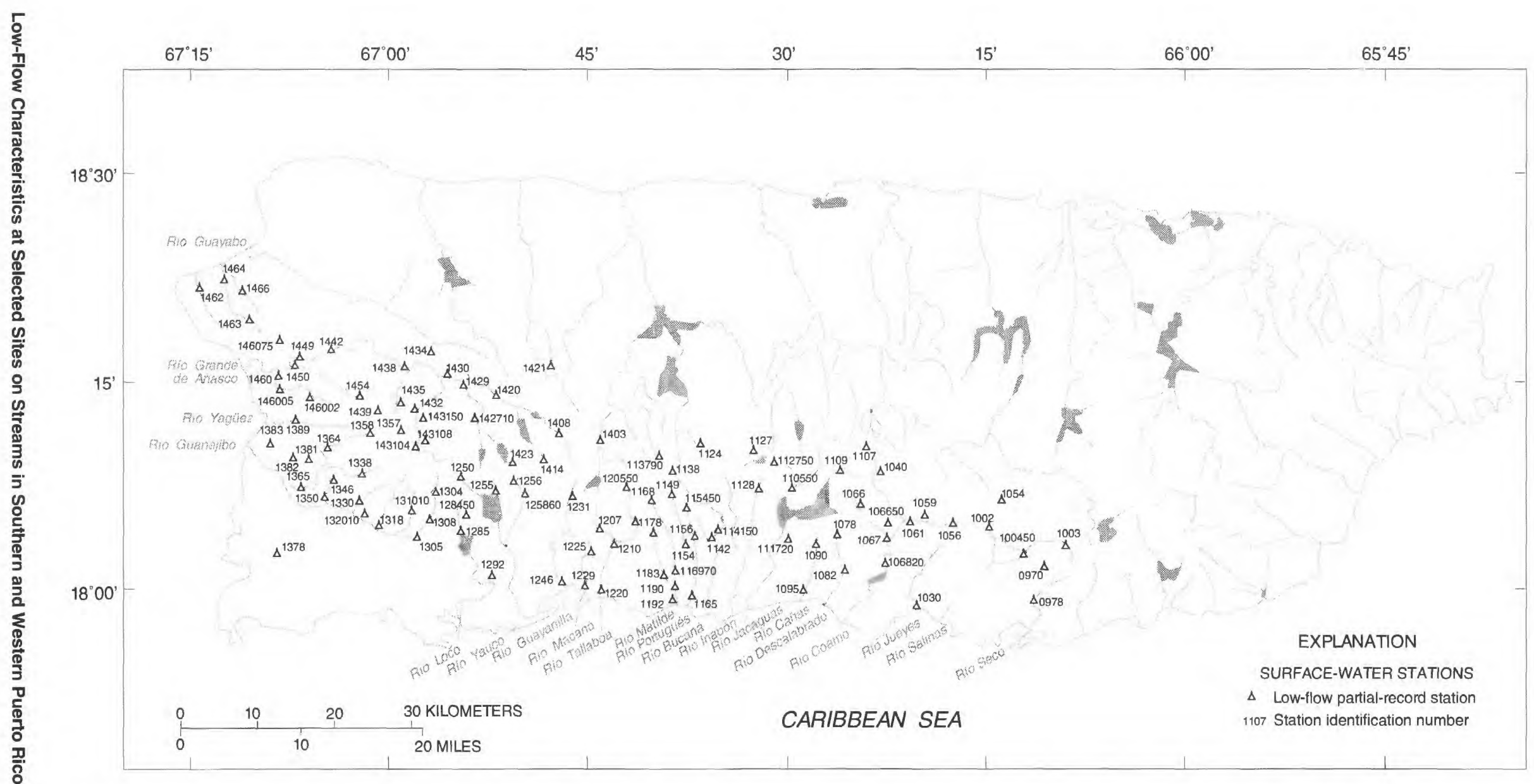

Figure 3. Location of low-flow partial-record gaging stations in southern and western Puerto Rico. Site numbers on map refer to the third through sixth or eighth digit of the station number shown in the station headings of appendix 2 . 
record (short-term continuous-record station) were analyzed as partial-record stations. Low-flow partialrecord stations are streamflow sites where periodic discharge measurements are made during different baseflow recessions over several years. Continuous- and partial-record stations were measured at least twice a year during base-flow periods for a total of eight discharge measurements per station in 4 years. The study includes low-flow frequency analyses for periods of 7-, 14-, 30-, 60-, and 90-consecutive days for recurrence intervals of 2 and 10 years at continuousrecord stations and estimates of low-flow frequency characteristics for 7-, 14-, and 30-consecutive days with recurrence intervals of 2 and 10 years at selected partialrecord stations.

\section{DESCRIPTION OF STUDY AREA}

The study area covers the southern and western parts of Puerto Rico from the town of Guayama in the southeast to the town of Aguada in the northwest (fig. 1). The south coastal area, extending from Guayama to Cabo Rojo and south of the Cordillera Central is the driest region in Puerto Rico. Located within this area is the Valle de Lajas, which has the lowest annual rainfall average (35 inches per year) in Puerto Rico.

The Cordillera Central mountain range is the most prominent topographic feature and the wettest (100 inches per year) region in the study area. This mountain range extends the entire length of Puerto Rico from the town of Yabucoa on the east coast to the town of Mayagüez on the west coast.

The Río Grande de Añasco with a drainage area about $204 \mathrm{mi}^{2}$ is the largest basin in southern and western Puerto Rico. Located within this basin are the Lago Yahuecas $\left(17 \mathrm{mi}^{2}\right)$, Lago Guayo $\left(9.6 \mathrm{mi}^{2}\right)$, and Lago Prieto $\left(9.6 \mathrm{mi}^{2}\right)$ reservoirs. The total drainage area of these reservoirs $\left(36.2 \mathrm{mi}^{2}\right)$ does not contribute any flow to the rest of the Río Grande de Añasco Basin during base-flow conditions. These reservoirs are connected by tunnels to divert water to the Lago Lucchetti hydroelectric plant in Yauco and the agricultural irrigation canal system in the south coast region. Most of river basins in the study area are characterized by turbulent streams and steep slopes that range from about 100 to about $1,000 \mathrm{ft} / \mathrm{mi}$ in the mountains to $10 \mathrm{ft} / \mathrm{mi}$ near the coast. Land-surface altitudes range from mean sea level to $4,020 \mathrm{ft}$ at Cerro La Puntita, which is the highest peak in Puerto Rico (fig. 1).

Southern and western Puerto Rico has a semiarid (south) to very wet (Cordillera Central) tropical climate with winds approaching predominately from the east. Average annual rainfall ranges from about 35 inches in the Valle de Lajas to about $\mathbf{1 0 0}$ inches in the Cordillera Central. Average annual pan evaporation of 60 inches was reported by the National Oceanic and Atmospheric Administration (NOAA) at the Valle de Lajas substation in Lajas from 1982 to 1986 (U.S. Department of Commerce, 1986). The dry season extends from January through April; the wet season extends from August through December. Normally, April is the driest month of the year. The minimum annual streamflow normally occurs during April or early May.

\section{METHODS OF ANALYSES}

Different techniques were applied to continuousand partial-record stations. Analyses for continuousrecord stations were based on frequency analyses of the annual minimum $n$-days low-flows. For partial-record stations, base-flow discharge measurements were related to concurrent base-flow discharge measurements or daily mean-flows at nearby continuous-record stations. The low-flow characteristics at the partial-record station were then determined through the correlation using the corresponding characteristics at the continuous-record station. A discussion of these techniques and the reliability of the estimates derived from them are provided in the following sections.

\section{Low Flow at Continuous-Record Gaging Stations}

Low-flow frequency curves were derived for nine continuous-record stations (fig. 2) using the method described by Riggs (1972) and by adaptation of the logPearson Type III flood-frequency program described by the Interagency Advisory Committee on Water Data (1982). The fitted log-Pearson Type III probability distribution and the recurrence interval (RI) of the annual $n$-day low flows were plotted by computer for each continuous-record station. Using a computer, discharges were arrayed in order of magnitude and 
assigned order numbers. The lowest discharge was given the order number 1 . The recurrence interval of each value in the array was computed using the plotting position formula currently in use by the USGS,

$$
\mathrm{RI}=(n+1) / \mathrm{m},
$$

where $n$ is the number of years of record and $\mathrm{m}$ is the order number in the array. The graphically-fitted curve based on the resulting plotting positions should be considered the basic frequency curve for annual low flows (Riggs, 1972). However, Riggs recommended that the computer plot be obtained and the log-Pearson Type III mathematical frequency curve (calculated value) be used if it is an adequate fit. For this report, the mathematical frequency curve was used because the mathematical and graphical frequency curves were similar, as shown by a typical computer plot (fig. 4).

Nine streamflow long-term continuous-record gaging stations (fig. 2) located on perennial streams with a minimum of 10 years of record were included in the analyses. The data used for these analyses have been published through the 1992 water year (U.S. Geological Survey Water Resources Data-Puerto Rico and the U.S. Virgin Islands, water years 1958-92).

The basic data requirement needed to determine low-flow frequency characteristics at a continuousrecord station is the annual minimum daily-mean discharge for selected lengths of time. In Puerto Rico, annual minimum discharge for streams usually occurs in April or early May. Frequency curves for each continuous-record gaging station were derived using the log-Pearson Type III distribution, adapted from the Interagency Advisory Committee on Water Data (1982). This study analyzed daily-mean discharge for continuous-record gaging stations for 7-, 14-, 30-, 60-, and 90-consecutive days in each water year (October 1 to September 30). Seven-consecutive days was the shortest period analyzed. Low-flow discharges were determined from the frequency curve for recurrence intervals of 2 and 10 years.

Low-flow characteristics of streams affected by reservoirs or irrigation canals may differ substantially from stations that exist under natural conditions, and data from regulated streams cannot be used to estimate the flow of nearby unregulated streams. Gaging stations where considerable anthropogenic regulation of stream flow existed were excluded from this study.

\section{Low Flow at Partial-Record Gaging Stations}

Low-flow characteristics for partial-record stations were estimated using a relation curve (fig. 5) developed by correlating base-flow discharge measurements made at partial-record stations with concurrent discharges at the continuous-record gaging stations (index stations) and the low-flow characteristic for the index station. This estimating technique, illustrated in figure 5 , transfers the low-flow characteristic for 7 -consecutive days at a 2 -year recurrence interval $\left(59.4 \mathrm{ft}^{3} / \mathrm{s}\right)$ computed by the logPearson Type III frequency distribution for the index station (50144000) to the graphically-determined relation curve to determine the corresponding low-flow characteristic (7-day, 2-year; $12.2 \mathrm{ft}^{3} / \mathrm{s}$ ) for the partialrecord station (50143200). Low-flow characteristics for 7-, 14-, and 30-consecutive days for recurrence intervals of 2 or 10 years were estimated as described above for 105 low-flow partial-record stations at which at least 8 low-flow discharge-measurements were available. The development of the control-point method (fig. 5) is described by Thomson and Carter (1963). This method is based on the observation that if correlation of simultaneous natural flows at gaging stations within short distances of each other were plotted on logarithmic coordinates, the relation curves tended to be straight lines which intersect the line of equal yield at a discharge about 1.5 times the average discharge at the independent station. The method is useful because the lower part of the relation curve can be consistently approximated by a straight line on a logarithmic plot. The relation curve must be defined by a sufficient number of simultaneous discharges. The line of equal yield (fig. 5) shows equal discharge per unit of drainage area and, in conjunction with the control-point method, helps establish the position of the relation curve which is used to estimate the low-flow frequency at the partial-record station. The analyses and compilations used in this report were made from USGS data files stored in Reston, Virginia.

All continuous-record index stations included in this report are located on perennial streams. Partialrecord stations were located as near as possible to corresponding index stations that were similar in size of drainage area and geologic setting. The number of baseflow discharge measurements and the index station number used in the correlations are included in the station headings of the partial-record stations table presented in appendix 2. 
RÍO GRANDE DE AÑASCO NEAR SAN SEBASTIÁN, PUERTO RICO

1964-92, 12-MONTH PERIOD ENDING SEPTEMBER 30

7-DAY LOW VALUE

PROBABILITY

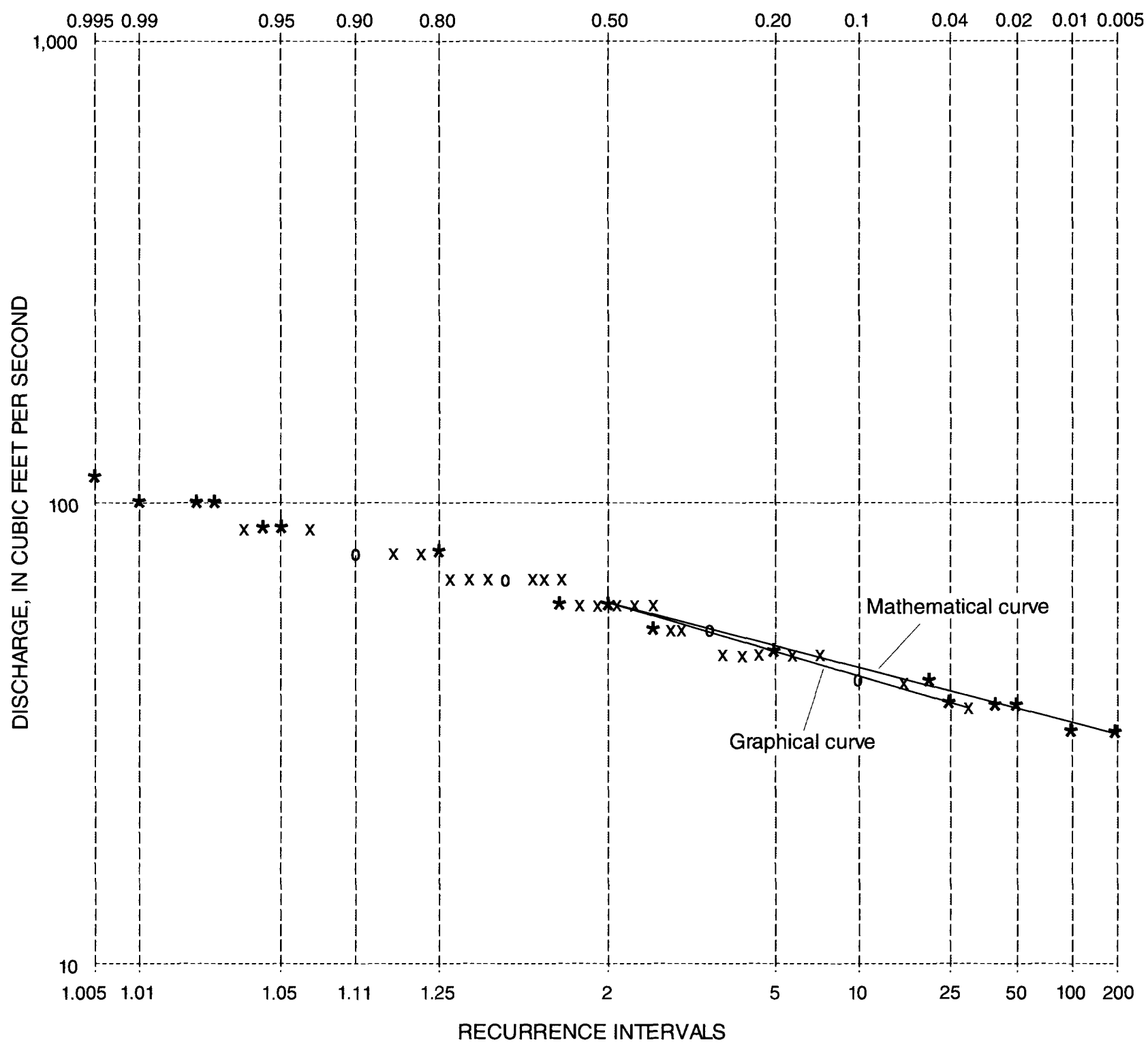

EXPLANATION

$x$ AN INPUT DATA VALUE

* a calculated value

- A CALCUlated VALUE AND ONE INPUT DATA VAlUe at SAME POSITION

$N$ YEARS OF RECORD AT STATION 50144000

Figure 4. Computer generated low-flow frequency curve and graphical low-flow frequency curve for the Río Grande de Añasco station near San Sebastián, Puerto Rico. 


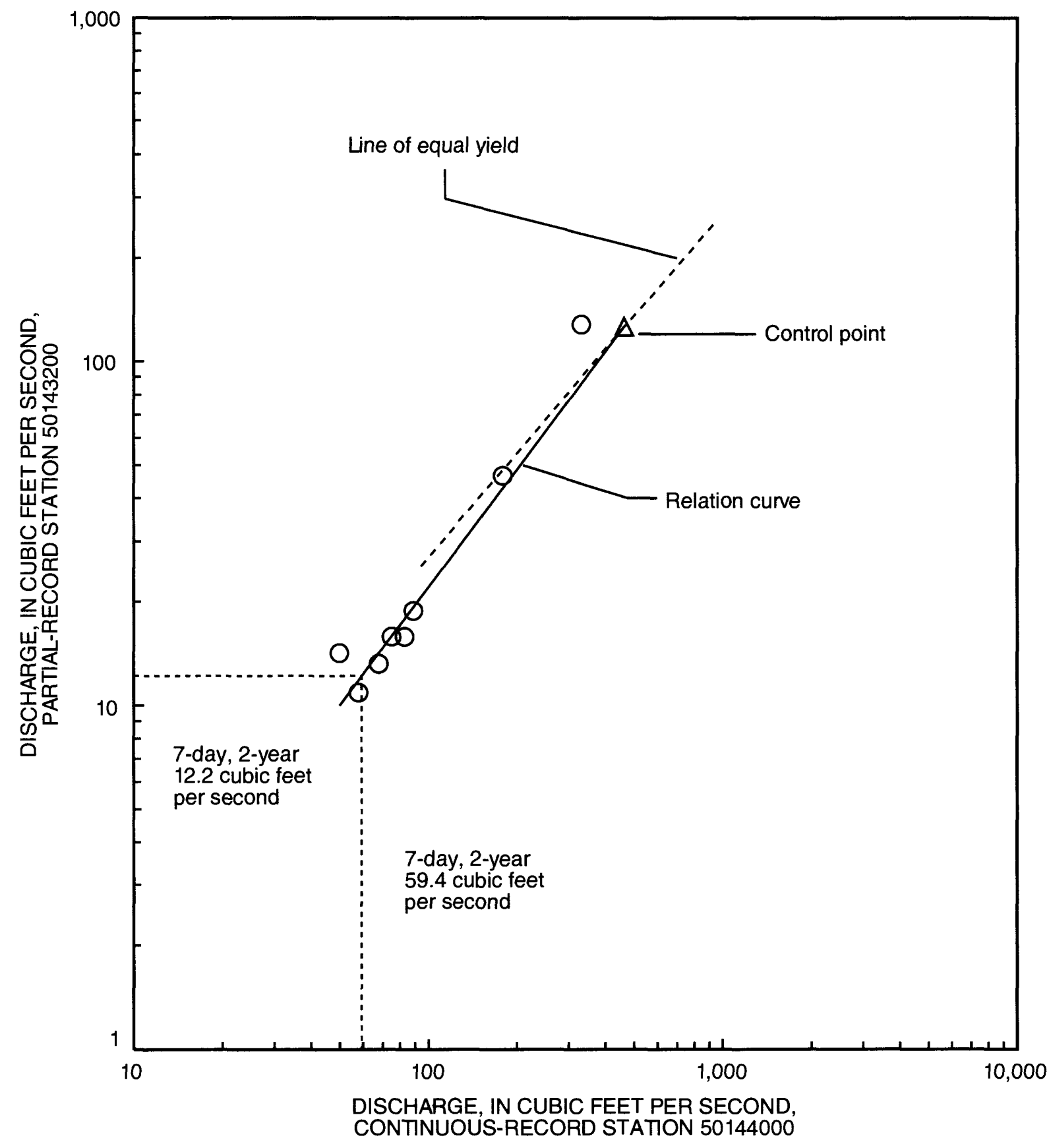

Figure 5. Relation between concurrent discharges at a partial-record station and a nearby continuous-record station. 


\section{Reliability of Results}

Low-flow frequency analyses are subject to errors. These errors are associated with many factors related to time, length of record, variability of flow, number of discharge measurements available for analyses, accuracy of discharge measurements, anthropogenic changes to streamflow (permanent or transitory), and compatibility of geologic features. The accuracy of estimates of low-flow characteristics is related to how accurately the correlation of concurrent discharges reflect the true relation between partial-record station and the index stations. The low-flow characteristics presented in this study for continuousrecord stations with less than 20 years of data will probably change to some degree as the length of record for these stations increases and more streamflow data are available for frequency analyses.

\section{PRESENTATION OF LOW-FLOW CHARACTERISTICS OF STREAMS}

Low-flow characteristics of streams are presented in appendices 1 and 2. Data for the 9 continuous-record gaging stations are presented first in appendix 1, followed by the data for the 105 partial-record stations in appendix 2. Low-flow characteristic values less than $0.1 \mathrm{ft}^{3} / \mathrm{s}$ are reported as $<0.1$. The heading for continuous-record gaging stations includes:

1. Location and description of the gaging site.

2. Drainage area.

3. Period of record analyzed.

4. Remarks.

The heading for partial-record gaging stations includes:

1. Location and description of the gaging site.

2. Drainage area.

3. Number of base-flow discharge measurements used in the correlations and the index gaging station number used.

4. Remarks.

\section{SUMMARY}

Low-flow frequency characteristics are essential in hydrologic studies for optimum development and utilization of streams. This report provides low-flow frequency analyses for 9 continuous-record streamflow gaging stations and 105 partial-record stations. Lowflow frequency characteristics for 7-, 14-, 30-, 60-, and 90-consecutive days for recurrence intervals of 2 and 10 years are presented for continuous-record stations. The log-Pearson Type III frequency distribution was used to analyze the annual $n$-day minimum streamflow records. Low flows for 7-, 14-, and 30-consecutive days for 2and 10-year recurrence intervals are presented for partial-record stations. This information was estimated by relating discharges at partial-record sites with concurrent discharges at nearby continuous-record stations with similar geologic, climatic, and topographic characteristics. Low-flow data from gaging stations where flows were affected by considerable anthropogenic regulation were excluded from the report. Factors such as the length of record, location of site, flow diversions, number of measurements, and the period during which the discharge was measured can affect the accuracy of the low-flow characteristics.

\section{REFERENCES}

Cobb, E.D., 1978, Estimates of 7-day, 10-year minimum flow at selected stream sites in Puerto Rico: U.S. Geological Survey Open-File Report 78-583, 47 p.

Colón-Dieppa, Eloy, and Quiñones-Aponte, Vicente, 1985, Estimates of 7-day, 10-year low flow at ungaged streams in Puerto Rico: U.S. Geological Survey WaterResources Investigations Report 84-4089, 1 pl.

Interagency Advisory Committee on Water Data, 1982, Guidelines for determining flood flow frequency: Bulletin 17B of the Hydrology Subcommittee, U.S. Geological Survey, Office of Water Data Coordination, Reston, Virginia.

Riggs, H.C., 1972, Low-flow investigations: U.S. Geological Survey Techniques of Water-Resources Investigations, book 4 , chapter B1, $18 \mathrm{p}$.

Santiago-Rivera, Luis, 1992, Low-flow characteristics at selected sites on streams in eastern Puerto Rico: U.S. Geological Survey Water-Resources Investigations Report 92-4063, 46 p.

Thomson, M.T., and Carter, R.F., 1963, Effect of a severe drought (1954) on streamflow in Georgia: Georgia Department of Natural Resources, Geologic and Water Resources Division Bulletin 73, 97 p.

U.S. Department of Commerce, 1986, Climatological data annual summary-Puerto Rico and the Virgin Islands: National Oceanic and Atmospheric Administration, v. 30 , no. $13,17 \mathrm{p}$

U.S. Geological Survey, 1958-92, Water resources data of Puerto Rico and the U.S. Virgin Islands, water years 1958-92: U.S. Geological Survey Water-Resources Data Reports (published annually). 


\section{APPENDIXES}




\section{APPENDIX 1. CONTINUOUS-RECORD STATIONS}

\section{RÍO GRANDE DE PATILLAS BASIN}

\section{Río Grande de Patillas near Patillas, Puerto Rico}

LOCATION.--Lat $18^{\circ} 02^{\prime} 04^{\prime \prime}$, long $66^{\circ} 01^{\prime} 58^{\prime \prime}$, Hydrologic Unit 21010004 , on left bank approximately $100 \mathrm{ft}$ above new bridge at Highway $184,1.2$ mi ( 1.9 $\mathrm{km})$ upstream from Lago Patillas Dam and $2.2 \mathrm{mi}$ ( $3.5 \mathrm{~km})$ northwest of Patillas.

DRAINAGE AREA.-- $18 \mathrm{mi}^{2}\left(47 \mathrm{~km}^{2}\right)$.

PERIOD OF RECORD ANALYZED.--February 1967 to September 1991.

REMARKS.--Minor diversion is made above the station for public-water supply.

MAGNITUDE AND FREQUENCY OF ANNUAL LOW FLOWS

\begin{tabular}{cccccc}
\hline $\begin{array}{c}\text { Recurrence } \\
\text { interval, }\end{array}$ & \multicolumn{5}{c}{$\begin{array}{c}\text { Lowest average flow, in cubic feet per second, } \\
\text { for indicated number of consecutive days }\end{array}$} \\
\cline { 2 - 6 } in years & $\mathbf{7}$ & $\mathbf{1 4}$ & $\mathbf{3 0}$ & $\mathbf{6 0}$ & $\mathbf{9 0}$ \\
\hline 2 & 10 & 11 & 14 & 17 & 20 \\
10 & 6.7 & 7.2 & 8.1 & 10 & 12 \\
\hline
\end{tabular}

RÍO INABÓN BASIN

50112500 Río Inabón at Real Abajo, Puerto Rico

LOCATION.--Lat $18^{\circ} 05^{\prime} 10^{\prime \prime}$, long 66³3'46", Hydrologic Unit 21010004, at bridge on private road, off Highway 511 at Hacienda La Concordia, 0.4 mi (0.6 $\mathrm{km}$ ) upstream from diversion canal, $0.5 \mathrm{mi}(0.8 \mathrm{~km})$ north of Real Abajo, and $6.1 \mathrm{mi}(9.8 \mathrm{~km})$ northeast of Plaza Degetau in Ponce.

DRAINAGE AREA.-- $9.7 \mathrm{mi}^{2}\left(25 \mathrm{~km}^{2}\right)$.

PERIOD OF RECORD ANALYZED.--February 1964 to September 1992.

REMARKS.--Minor diversion is made above the station for public-water supply.

MAGNITUDE AND FREQUENCY OF ANNUAL LOW FLOWS

\begin{tabular}{cccccc}
\hline $\begin{array}{c}\text { Recurrence } \\
\text { interval, }\end{array}$ & \multicolumn{5}{c}{$\begin{array}{c}\text { Lowest average flow, in cubic feet per second, } \\
\text { for indicated number of consecutive days }\end{array}$} \\
\cline { 2 - 6 } in years & $\mathbf{7}$ & $\mathbf{1 4}$ & $\mathbf{3 0}$ & $\mathbf{6 0}$ & $\mathbf{9 0}$ \\
\hline 2 & 2.5 & 2.9 & 3.4 & 4.2 & 4.7 \\
10 & 1.6 & 1.8 & 2.2 & 2.6 & 3.0 \\
\hline
\end{tabular}

RÍO BUCANÁ BASIN

\section{Río Cerrillos near Ponce, Puerto Rico}

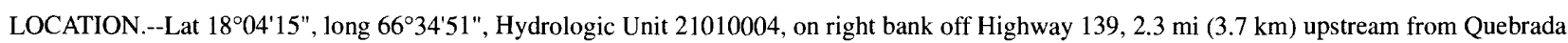
Ausubo, and $4.6 \mathrm{mi}(7.4 \mathrm{~km})$ northeast from Plaza Degetau in Ponce.

DRAINAGE AREA.-- $18 \mathrm{mi}^{2}\left(46 \mathrm{~km}^{2}\right)$.

PERIOD OF RECORD ANALYZED.--May 1964 to September 1992.

REMARKS.--Considerable flow regulation by Lago Cerrillos Dam since March 1993. Low-flow data used in the correlations were collected prior to the regulation date.

MAGNITUDE AND FREQUENCY OF ANNUAL LOW FLOWS

\begin{tabular}{cccccc}
\hline $\begin{array}{c}\text { Recurrence } \\
\text { interval, }\end{array}$ & \multicolumn{5}{c}{$\begin{array}{c}\text { Lowest average flow, in cubic feet per second, } \\
\text { for indicated number of consecutive days }\end{array}$} \\
\cline { 2 - 6 } in years & $\mathbf{7}$ & $\mathbf{1 4}$ & $\mathbf{3 0}$ & $\mathbf{6 0}$ & $\mathbf{9 0}$ \\
\hline & & & & & \\
2 & 4.9 & 5.3 & 6.4 & 7.5 & 8.6 \\
10 & 2.8 & 3.4 & 4.3 & 4.9 & 5.6 \\
\hline
\end{tabular}




\section{RÍO PORTUGUÉS BASIN}

\section{Río Portugués near Ponce, Puerto Rico}

LOCATION.--Lat $18^{\circ} 04^{\prime} 45^{\prime \prime}$, long 66 $38^{\prime} 01^{\prime \prime}$, Hydrologic Unit 21010004 , on right bank $30 \mathrm{ft}(9 \mathrm{~m})$ upstream from bridge on Highway $504,0.2 \mathrm{mi}(0.3 \mathrm{~km})$ upstream from small unnamed tributary, $4.4 \mathrm{mi}(7.1 \mathrm{~km})$ upstream from Río Chiquito, and $4.7 \mathrm{mi}(7.6 \mathrm{~km})$ north of Plaza Degetau in Ponce.

DRAINAGE AREA.--8.8 $\mathrm{mi}^{2}\left(23 \mathrm{~km}^{2}\right)$.

PERIOD OF RECORD ANALYZED.--July 1964 to September 1992.

REMARKS.--Minor diversion is made above the station for public-water supply.

MAGNITUDE AND FREQUENCY OF ANNUAL LOW FLOWS

\begin{tabular}{|c|c|c|c|c|c|}
\hline \multirow{2}{*}{$\begin{array}{c}\text { Recurrence } \\
\text { interval, } \\
\text { in years }\end{array}$} & \multicolumn{5}{|c|}{$\begin{array}{l}\text { Lowest average flow, in cubic feet per second, } \\
\text { for indicated number of consecutive days }\end{array}$} \\
\hline & 7 & 14 & 30 & 60 & 90 \\
\hline $\begin{array}{r}2 \\
10\end{array}$ & $\begin{array}{l}2.6 \\
1.7\end{array}$ & $\begin{array}{l}2.8 \\
1.9\end{array}$ & $\begin{array}{l}3.3 \\
2.2\end{array}$ & $\begin{array}{l}4.0 \\
2.6\end{array}$ & $\begin{array}{l}4.7 \\
3.0\end{array}$ \\
\hline
\end{tabular}

\section{RÍO GUAYANILLA BASIN}

\section{Río Guayanilla near Guayanilla, Puerto Rico}

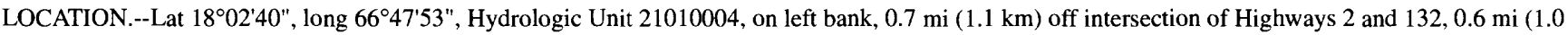
$\mathrm{km})$ downstream from Quebrada Consejo, $1.8 \mathrm{mi}(2.9 \mathrm{~km})$ north-northwest from Plaza de Guayanilla.

DRAINAGE AREA.-- $19 \mathrm{mi}^{2}\left(49 \mathrm{~km}^{2}\right)$.

PERIOD OF RECORD ANALYZED.--March 1981 to September 1992.

REMARKS.--Minor diversion is made above the station to filter plant for public-water supply.

MAGNITUDE AND FREQUENCY OF ANNUAL LOW FLOWS

\begin{tabular}{cccccc}
\hline $\begin{array}{c}\text { Recurrence } \\
\text { interval, }\end{array}$ & \multicolumn{5}{c}{$\begin{array}{c}\text { Lowest average flow, in cubic feet per second, } \\
\text { for indicated number of consecutive days }\end{array}$} \\
\cline { 2 - 6 } in years & $\mathbf{7}$ & $\mathbf{1 4}$ & $\mathbf{3 0}$ & $\mathbf{6 0}$ & $\mathbf{9 0}$ \\
\hline 2 & 3.2 & 3.6 & 4.1 & 5.4 & 6.5 \\
10 & 2.4 & 2.9 & 3.3 & 4.3 & 5.0 \\
\hline
\end{tabular}

\section{RÍO GUANAJIBO BASIN \\ 50136000 Río Rosario at Rosario, Puerto Rico}

LOCATION.--Lat $18^{\circ} 10^{\prime} 22^{\prime \prime}$, long 6704'31", Hydrologic Unit 21010003 , on left bank above low dam, $0.2 \mathrm{mi}(0.3 \mathrm{~km})$ below Quebrada Figueroa, $0.7 \mathrm{mi}$

$(1.1 \mathrm{~km})$ northeast of Rosario, and $1.6 \mathrm{mi}(2.6 \mathrm{~km})$ below Quebrada Palma.

DRAINAGE AREA.-- $16.4 \mathrm{mi}^{2}\left(42.5 \mathrm{~km}^{2}\right)$.

PERIOD OF RECORD ANALYZED.--June 1961 to September 1986.

REMARKS.--Minor diversions are made above the station to filter plant for public-water supply.

MAGNITUDE AND FREQUENCY OF ANNUAL LOW FLOWS

\begin{tabular}{cccccc}
\hline $\begin{array}{c}\text { Recurrence } \\
\text { interval, } \\
\text { in years }\end{array}$ & $\mathbf{7}$ & \multicolumn{4}{c}{$\begin{array}{c}\text { Lowest average flow, in cubic feet per second, } \\
\text { for indicated number of consecutive days }\end{array}$} \\
\hline 2 & 14 & $\mathbf{3 0}$ & $\mathbf{6 0}$ & $\mathbf{9 0}$ \\
\hline 10 & 10 & 11 & 11 & 12 & 14 \\
& 5.4 & 5.8 & 6.7 & 7.7 & 8.8 \\
\hline
\end{tabular}




\section{RÍO GUANAJIBO BASIN-Continued}

\section{Río Guanajibo near Hormigueros, Puerto Rico}

LOCATION.--Lat $18^{\circ} 08^{\prime} 36^{\prime \prime}$, long $67^{\circ} 08^{\prime} 57^{\prime \prime}$, Hydrologic Unit 21010003 , at bridge on Highway $100,1.4 \mathrm{mi}(2.3 \mathrm{~km})$ west of Hormigueros, and $2.0 \mathrm{mi}(3.2$ $\mathrm{km})$ downstream from Río Rosario.

DRAINAGE AREA.--120 $\mathrm{mi}^{2}\left(311 \mathrm{~km}^{2}\right)$.

PERIOD OF RECORD ANALYZED.--April 1974 to September 1992.

REMARKS.--Minor diversions are made above the station to filter plant for public-water supply.

MAGNITUDE AND FREQUENCY OF ANNUAL LOW FLOWS

\begin{tabular}{cccccc}
\hline $\begin{array}{c}\text { Recurrence } \\
\text { interval, } \\
\text { in years }\end{array}$ & $\mathbf{7}$ & \multicolumn{4}{c}{$\begin{array}{c}\text { Lowest average flow, in cubic feet per second, } \\
\text { for indicated number of consecutive days }\end{array}$} \\
\hline 2 & 16 & $\mathbf{1 4}$ & $\mathbf{3 0}$ & $\mathbf{6 0}$ & $\mathbf{9 0}$ \\
\hline 10 & 9.0 & 20 & 23 & 29 & 35 \\
\end{tabular}

RÍO GRANDE DE AÑASCO BASIN

50141000 Río Blanco near Adjuntas, Puerto Rico

LOCATION.--Lat $18^{\circ} 12^{\prime} 19^{\prime \prime}$, long $66^{\circ} 48^{\prime} 01$ ", Hydrologic Unit 21010003 , on right bank near dirt road off Highway $129,0.4$ mi $(0.6 \mathrm{~km})$ north-northwest from intersection of Highways 135 and 129, $2.5 \mathrm{mi}(4 \mathrm{~km})$ northeast of Castañer, $2.3 \mathrm{mi}(3.7 \mathrm{~km})$ east-southeast of Lago Guayo Dam, and $0.5 \mathrm{mi}(0.8 \mathrm{~km})$ downstream from Río Limani.

DRAINAGE AREA. $-15 \mathrm{mi}^{2}\left(40 \mathrm{~km}^{2}\right)$.

PERIOD OF RECORD ANALYZED.--March 1946 to September 1984.

MAGNITUDE AND FREQUENCY OF ANNUAL LOW FLOWS

\begin{tabular}{cccccc}
\hline $\begin{array}{c}\text { Recurrence } \\
\text { interval, }\end{array}$ & \multicolumn{5}{c}{$\begin{array}{c}\text { Lowest average flow, in cubic feet per second, } \\
\text { for indicated number of consecutive days }\end{array}$} \\
\cline { 2 - 6 } in years & $\mathbf{7}$ & $\mathbf{1 4}$ & $\mathbf{3 0}$ & $\mathbf{6 0}$ & $\mathbf{9 0}$ \\
\hline 2 & 8.5 & 9.0 & 10 & 12 & 13 \\
10 & 6.3 & 6.7 & 7.5 & 8.6 & 9.4 \\
\hline
\end{tabular}

50144000 Río Grande de Añasco near San Sebastián, Puerto Rico

LOCATION.--Lat $18^{\circ} 17^{\prime} 05^{\prime \prime}$, long $67^{\circ} 03^{\prime} 05^{\prime \prime}$, Hydrologic Unit 21010003 , on left bank, approximately $100 \mathrm{ft}(30.5 \mathrm{~m})$ below bridge of Highway $108,0.4 \mathrm{mi}$ $(0.6 \mathrm{~km})$ downstream from Quebrada La Zumbadora, $4.4 \mathrm{mi}(7.1 \mathrm{~km})$ northwest of Las Marías, $5.4 \mathrm{mi}(8.7 \mathrm{~km})$ southwest of San Sebastián.

DRAINAGE AREA.--94 mi ${ }^{2}\left(244 \mathrm{~km}^{2}\right)$, does not include $36.2 \mathrm{mi}^{2}\left(93.8 \mathrm{~km}^{2}\right)$ which base flow is diverted to Lago Lucchetti Dam.

PERIOD OF RECORD ANALYZED.--August 1964 to September 1992.

REMARKS.--Flow is regulated by Lago Yahuecas, Lago Guayo and Lago Prieto Dams. The total drainage area of these reservoirs $\left(36.2 \mathrm{mi}^{2}\right) \mathrm{does}$ not contribute any flow to the rest of the Río Grande de Añasco Basin during base-flow conditions. Minor diversions are made above the station for public-water supply.

MAGNITUDE AND FREQUENCY OF ANNUAL LOW FLOWS

\begin{tabular}{cccccc}
\hline $\begin{array}{c}\text { Recurrence } \\
\text { interval, } \\
\text { in years }\end{array}$ & $\mathbf{7}$ & \multicolumn{4}{c}{$\begin{array}{l}\text { Lowest average flow, in cubic feet per second, } \\
\text { for indicated number of consecutive days }\end{array}$} \\
\cline { 2 - 6 } & $\mathbf{1 4}$ & $\mathbf{3 0}$ & $\mathbf{6 0}$ & $\mathbf{9 0}$ \\
\hline 2 & 59 & 64 & 71 & 84 & 95 \\
10 & 43 & 46 & 54 & 62 & 70 \\
\hline
\end{tabular}




\section{APPENDIX 2. PARTIAL-RECORD STATIONS}

\section{RÍO SECO BASIN}

\section{Quebrada Cimarrona near Jobos, Puerto Rico}

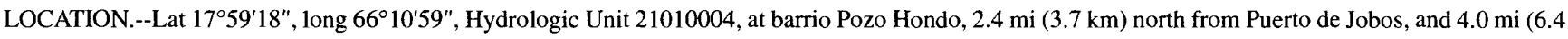
$\mathrm{km})$ northwest from Plaza de Guayama.

DRAINAGE AREA.--3.1 $\mathrm{mi}^{2}\left(8.0 \mathrm{~km}^{2}\right)$.

REMARKS.--Estimated minimum average 7-, 14-, and 30-day flow with a 2- and 10-year recurrence interval is 0.0 on the basis of 6 observations of zero flow under base-flow conditions.

\section{Río Seco near Central Guamaní, Puerto Rico}

LOCATION.--Lat $17^{\circ} 58^{\prime} 06^{\prime \prime}$, long $66^{\circ} 10^{\prime} 52^{\prime \prime}$, Hydrologic Unit 21010004 , at bridge on Highway 3, $0.2 \mathrm{mi}(0.3 \mathrm{~km})$ north of Central Guamaní, and $1.2 \mathrm{mi}$ $(1.9 \mathrm{~km})$ northwest of Jobos.

DRAINAGE AREA.--11 $\mathrm{mi}^{2}\left(29 . \mathrm{km}^{2}\right)$.

REMARKS.--Estimated minimum average 7-, 14-, and 30-day flow with a 2- and 10-year recurrence interval is 0.0 on the basis of 8 observations of zero flow under base-flow conditions.

\section{RÍO SALINAS BASIN}

\section{Río Lapa near Rabo del Buey, Puerto Rico}

LOCATION.--Lat 1803'36", long 66 14'28", Hydrologic Unit 21010004, at barrio Lapa, on Highway 1, $1.6 \mathrm{mi}$ (2.6 km) upstream from confluence with Río Majada, and $6.2 \mathrm{mi}(10 \mathrm{~km})$ southwest from Plaza de Cayey.

DRAINAGE AREA.-- $10 \mathrm{mi}^{2}\left(26 \mathrm{~km}^{2}\right)$.

LOW-FLOW ESTIMATES.--Based on correlation of 8 base-flow measurements with concurrent base flows at gaging station 50092000. MAGNITUDE AND FREQUENCY OF ANNUAL LOW FLOWS

\begin{tabular}{cccc}
\hline $\begin{array}{c}\text { Recurrence } \\
\text { interval, }\end{array}$ & \multicolumn{2}{c}{$\begin{array}{c}\text { Lowest average flow, in cubic feet per second, } \\
\text { for indicated number of consecutive days }\end{array}$} \\
\cline { 2 - 4 } in years & $\mathbf{7}$ & $\mathbf{1 4}$ & $\mathbf{3 0}$ \\
\hline 2 & .4 & .5 & .7 \\
10 & .2 & .2 & .2 \\
\hline
\end{tabular}

50100300 Río Jájome at Jájome, Puerto Rico

LOCATION.--Lat 1803'49", long 6609'38", Hydrologic Unit 21010004, at barrio Jájome Bajo, on Highway 708, 3.5 mi (5.6 km) south from Plaza de Cayey.

DRAINAGE AREA.-- $4.6 \mathrm{mi}^{2}\left(12 \mathrm{~km}^{2}\right)$.

LOW-FLOW ESTIMATES.--Based on correlation of 8 base-flow measurements with concurrent base flows at gaging station 50092000 . MAGNITUDE AND FREQUENCY OF ANNUAL LOW FLOWS

\begin{tabular}{cccc}
\hline $\begin{array}{c}\text { Recurrence } \\
\text { interval, } \\
\text { in years }\end{array}$ & \multicolumn{2}{c}{$\begin{array}{c}\text { Lowest average flow, in cubic feet per second, } \\
\text { for indicated number of consecutive days }\end{array}$} \\
\cline { 2 - 4 } & $\mathbf{7}$ & $\mathbf{1 4}$ & $\mathbf{3 0}$ \\
\hline 2 & .4 & .5 & .7 \\
10 & .2 & .2 & .2 \\
\hline
\end{tabular}


RÍO SALINAS BASIN-Continued

50100450 Río Majada at La Plena, Puerto Rico

LOCATION.--Lat $18^{\circ} 02^{\prime} 40^{\prime \prime}$, long $66^{\circ} 12^{\prime} 27^{\prime \prime}$, Hydrologic Unit 21010004, at barrio Quebrada Yegua, at Highway $712,2.0 \mathrm{mi}(3.2 \mathrm{~km})$ northeast from Albergue Olímpico, and $5.5 \mathrm{mi}(8.8 \mathrm{~km})$ southwest from Plaza de Cayey.

DRAINAGE AREA.--17 $\mathrm{mi}^{2}\left(43 \mathrm{~km}^{2}\right)$.

LOW-FLOW ESTIMATES.--Based on correlation of 8 base-flow measurements with concurrent base flows at gaging station 50092000.

MAGNITUDE AND FREQUENCY OF ANNUAL LOW FLOWS

\begin{tabular}{cccc}
\hline $\begin{array}{c}\text { Recurrence } \\
\text { interval, }\end{array}$ & \multicolumn{2}{c}{$\begin{array}{c}\text { Lowest average flow, in cubic feet per second, } \\
\text { for indicated number of consecutive days }\end{array}$} \\
\cline { 2 - 4 } in years & $\mathbf{7}$ & $\mathbf{1 4}$ & $\mathbf{3 0}$ \\
\hline 2 & .5 & .6 & 1.0 \\
10 & .2 & .2 & .3 \\
\hline
\end{tabular}

RÍO JUEYES BASIN

50103000 Río Jueyes near Jauca, Puerto Rico

LOCATION.--17 $58^{\prime} 45^{\prime \prime}$, long $66^{\circ} 20^{\prime} 20^{\prime \prime}$, Hydrologic Unit 21010004 , at bridge on Highway $1,1.8 \mathrm{mi}(2.9 \mathrm{~km})$ east of Jaucas, and $2.7 \mathrm{mi}(4.3 \mathrm{~km})$ west of Plaza de Salinas.

DRAINAGE AREA.--8.6 $\mathrm{mi}^{2}\left(22 \mathrm{~km}^{2}\right)$.

REMARKS.--Estimated minimum average 7-, 14-, and 30-day flow with a 2- and 10-year recurrence interval is 0.0 on the basis of 8 observations of zero flow under base-flow conditions.

\section{RÍO COAMO BASIN}

\section{Río Coamo near Pasto, Puerto Rico}

LOCATION.--Lat 1807'08", long 66² 21'52", Hydrologic Unit 21010004, at barrio Pasto, on Highway 555, 2.6 mi (4.2 km) northwest from Plaza de Coamo.

DRAINAGE AREA.-- $9.0 \mathrm{mi}^{2}\left(23 \mathrm{~km}^{2}\right)$.

LOW-FLOW ESTIMATES.--Based on correlation of 8 base-flow measurements with concurrent base flows at gaging station 50112500 .

MAGNITUDE AND FREQUENCY OF ANNUAL LOW FLOWS

\begin{tabular}{cccc}
\hline $\begin{array}{c}\text { Recurrence } \\
\text { interval, }\end{array}$ & \multicolumn{2}{c}{$\begin{array}{c}\text { Lowest average flow, in cubic feet per second, } \\
\text { for indicated number of consecutive days }\end{array}$} \\
\cline { 2 - 4 } in years & $\mathbf{7}$ & $\mathbf{1 4}$ & $\mathbf{3 0}$ \\
\hline 2 & 2.9 & 3.4 & 4.0 \\
10 & 2.0 & 2.2 & 2.6 \\
\hline
\end{tabular}

50105400 Río Cuyón at La Guava, Puerto Rico

LOCATION.--Lat $18^{\circ} 05^{\prime \prime} 20^{\prime \prime}$, long 66 $16^{\prime} 17^{\prime \prime}$, Hydrologic Unit 21010004, at barrio Algarrobo, on Highway 717, $1.0 \mathrm{mi}$ (1.6 km) southwest from Cerro Verdún, and $5.6 \mathrm{mi}(9.0 \mathrm{~km})$ east from Plaza de Coamo.

DRAINAGE AREA.-- $4.3 \mathrm{mi}^{2}\left(11 \mathrm{~km}^{2}\right)$.

LOW-FLOW ESTIMATES.--Based on correlation of 8 base-flow measurements with concurrent base flows at gaging station 50112500 .

MAGNITUDE AND FREQUENCY OF ANNUAL LOW FLOWS

\begin{tabular}{cccc}
\hline $\begin{array}{c}\text { Recurrence } \\
\text { interval, }\end{array}$ & \multicolumn{2}{c}{$\begin{array}{c}\text { Lowest average flow, in cubic feet per second, } \\
\text { for indicated number of consecutive days }\end{array}$} \\
\cline { 2 - 4 } in years & $\mathbf{7}$ & $\mathbf{1 4}$ & $\mathbf{3 0}$ \\
\hline 2 & $<.1$ & $<.1$ & $<1$ \\
10 & $<.1$ & $<.1$ & $<.1$ \\
\hline
\end{tabular}




\section{RÍO COAMO BASIN-Continued}

\section{Río Cuyón near Coamo, Puerto Rico}

LOCATION.--Lat $18^{\circ} 05^{\prime} 25^{\prime \prime}$, long 66² 18'50", Hydrologic Unit 21010004, at barrio Cuyón, on Highway 14, 0.8 mi (1.3 km) southeast from Cerro Santa Ana, and $2.8 \mathrm{mi}(4.5 \mathrm{~km})$ northeast from Plaza de Coamo.

DRAINAGE AREA.-- $18 \mathrm{mi}^{2}\left(47 \mathrm{~km}^{2}\right)$.

LOW-FLOW ESTIMATES.--Based on correlation of 8 base-flow measurements with concurrent base flows at gaging station 50112500 . MAGNITUDE AND FREQUENCY OF ANNUAL LOW FLOWS

\begin{tabular}{cccc}
\hline $\begin{array}{c}\text { Recurrence } \\
\text { interval, } \\
\text { in years }\end{array}$ & \multicolumn{2}{c}{$\begin{array}{c}\text { Lowest average flow, in cubic feet per second, } \\
\text { for indicated number of consecutive days }\end{array}$} \\
\cline { 2 - 4 } & $\mathbf{7}$ & $\mathbf{1 4}$ & $\mathbf{3 0}$ \\
\hline 2 & .5 & .7 & 1.0 \\
10 & .2 & .3 & .4 \\
\hline
\end{tabular}

\section{Quebrada Montería near Coamo, Puerto Rico}

LOCATION.--Lat $18^{\circ} 05^{\prime} 13^{\prime \prime}$, long 66²1'04", Hydrologic Unit 21010004, at barrio Pasto, at confluence with Río Cuyón, and $0.5 \mathrm{mi}(0.8 \mathrm{~km})$ northeast from Plaza de Coamo.

DRAINAGE AREA.--7.1 $\mathrm{mi}^{2}\left(18 \mathrm{~km}^{2}\right)$.

LOW-FLOW ESTIMATES.--Based on correlation of 8 base-flow measurements with concurrent base flows at gaging station 50112500 .

MAGNITUDE AND FREQUENCY OF ANNUAL LOW FLOWS

\begin{tabular}{cccc}
\hline $\begin{array}{c}\text { Recurrence } \\
\text { interval, }\end{array}$ & \multicolumn{2}{c}{$\begin{array}{c}\text { Lowest average flow, in cubic feet per second, } \\
\text { for indicated number of consecutive days }\end{array}$} \\
\cline { 2 - 4 } in years & $\mathbf{7}$ & $\mathbf{1 4}$ & $\mathbf{3 0}$ \\
\hline 2 & .1 & .2 & .2 \\
10 & $<.1$ & $<.1$ & $<.1$ \\
\hline
\end{tabular}

50106100 Río Coamo at Coamo, Puerto Rico

LOCATION.--Lat $18^{\circ} 05^{\prime} 00^{\prime \prime}$, long 66²1'16", Hydrologic Unit 21010004, at Coamo, on Highway 14, $500 \mathrm{ft}$ (152 m) downstream from confluence with Río Cuyón, and $0.2 \mathrm{mi}(0.3 \mathrm{~km})$ east from Plaza de Coamo.

DRAINAGE AREA.-- $44 \mathrm{mi}^{2}\left(113 \mathrm{~km}^{2}\right)$.

LOW-FLOW ESTIMATES.--Based on correlation of 9 base-flow measurements with concurrent base flows at gaging station 50112500 .

REMARKS.--Minor diversions are made above station to filter plant for public-water supply.

MAGNITUDE AND FREQUENCY OF ANNUAL LOW FLOWS

\begin{tabular}{cccc}
\hline $\begin{array}{c}\text { Recurrence } \\
\text { interval, }\end{array}$ & \multicolumn{2}{c}{$\begin{array}{c}\text { Lowest average flow, in cubic feet per second, } \\
\text { for indicated number of consecutive days }\end{array}$} \\
\cline { 2 - 4 } in years & $\mathbf{7}$ & $\mathbf{1 4}$ & $\mathbf{3 0}$ \\
\hline 2 & 2.0 & 2.6 & 3.6 \\
10 & .9 & 1.2 & 1.6 \\
\hline
\end{tabular}

50106600 Río de La Mina near Coamo, Puerto Rico

LOCATION.--Lat $18^{\circ} 05^{\prime} 04^{\prime \prime}$, long 66²3'22", Hydrologic Unit 21010004, at barrio Santa Catalina, on Highway 150, $2.2 \mathrm{mi}$ (3.5 km) west from Plaza de Coamo.

DRAINAGE AREA.--2.6 $\mathrm{mi}^{2}\left(6.8 \mathrm{~km}^{2}\right)$.

LOW-FLOW ESTIMATES.--Based on correlation of 8 base-flow measurements with concurrent base flows at gaging station 50112500. MAGNITUDE AND FREQUENCY OF ANNUAL LOW FLOWS

\begin{tabular}{cccc}
\hline $\begin{array}{c}\text { Recurrence } \\
\text { interval, } \\
\text { in years }\end{array}$ & \multicolumn{2}{c}{$\begin{array}{c}\text { Lowest average flow, in cubic feet per second, } \\
\text { for indicated number of consecutive days }\end{array}$} \\
\cline { 2 - 4 } & $\mathbf{7}$ & $\mathbf{1 4}$ & $\mathbf{3 0}$ \\
\hline 2 & $<.1$ & $<.1$ & .1 \\
10 & $<.1$ & $<.1$ & $<.1$ \\
\hline
\end{tabular}




\section{RÍO COAMO BASIN-Continued}

\section{Río del Pasto near Coamo, Puerto Rico}

LOCATION.--Lat $18^{\circ} 04^{\prime} 49^{\prime \prime}$, long 66²2'32", Hydrologic Unit 21010004, at barrio San Idelfonso, on Highway $150,1.3 \mathrm{mi}(2.1 \mathrm{~km})$ west from Plaza de Coamo.

DRAINAGE AREA.-- $1.8 \mathrm{mi}^{2}\left(4.7 \mathrm{~km}^{2}\right)$

LOW-FLOW ESTIMATES.--Based on correlation of 8 base-flow measurements with concurrent low flows at gaging station 50112500 .

MAGNITUDE AND FREQUENCY OF ANNUAL LOW FLOWS

\begin{tabular}{cccc}
\hline $\begin{array}{c}\text { Recurrence } \\
\text { interval, } \\
\text { in years }\end{array}$ & \multicolumn{2}{c}{$\begin{array}{c}\text { Lowest average flow, in cubic feet per second, } \\
\text { for indicated number of consecutive days }\end{array}$} \\
\cline { 2 - 4 } & $\mathbf{7}$ & $\mathbf{1 4}$ & $\mathbf{3 0}$ \\
\hline 2 & $<.1$ & $<.1$ & .1 \\
10 & $<.1$ & $<.1$ & $<.1$ \\
\hline
\end{tabular}

50106700 Río de La Mina at Coamo, Puerto Rico

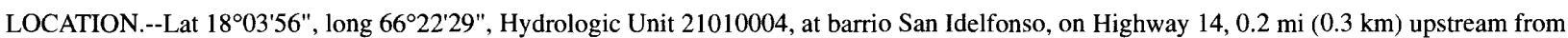
confluence with Río Coamo, and $1.7 \mathrm{mi}(2.7 \mathrm{~km})$ from Plaza de Coamo.

DRAINAGE AREA.--5.9 $\mathrm{mi}^{2}\left(15 \mathrm{~km}^{2}\right)$.

LOW-FLOW ESTIMATES.--Based on correlation of 8 base-flow measurements with concurrent base flows at gaging station 50112500.

MAGNITUDE AND FREQUENCY OF ANNUAL LOW FLOWS

\begin{tabular}{cccc}
\hline $\begin{array}{c}\text { Recurrence } \\
\text { interval, } \\
\text { in years }\end{array}$ & \multicolumn{2}{c}{$\begin{array}{c}\text { Lowest average flow, in cubic feet per second, } \\
\text { for indicated number of consecutive days }\end{array}$} \\
\cline { 2 - 4 } & \multicolumn{7}{|c}{} & $\mathbf{1 4}$ & $\mathbf{3 0}$ \\
\hline 2 & $<.1$ & .1 & .2 \\
10 & $<.1$ & $<.1$ & $<.1$ \\
\hline
\end{tabular}

50106820 Río Coamo at Baños de Coamo, Puerto Rico

LOCATION.--Lat $18^{\circ} 02^{\prime 2} 3^{\prime \prime}$, long $66^{\circ} 22^{\prime} 31^{\prime \prime}$, Hydrologic Unit 21010004, at barrio San Idelfonso, at the end of Highway $546,3.3 \mathrm{mi}(5.3 \mathrm{~km})$ southwest from Plaza de Coamo.

DRAINAGE AREA.--58 $\mathrm{mi}^{2}\left(152 \mathrm{~km}^{2}\right)$.

LOW-FLOW ESTIMATES.--Based on correlation of 9 base-flow measurements with concurrent base flows at gaging station 50112500.

REMARKS.--Minor diversions are made above station to filter plant for public-water supply.

MAGNITUDE AND FREQUENCY OF ANNUAL LOW FLOWS

\begin{tabular}{cccc}
\hline $\begin{array}{c}\text { Recurrence } \\
\text { interval, }\end{array}$ & \multicolumn{2}{c}{$\begin{array}{c}\text { Lowest average flow, in cubic feet per second, } \\
\text { for indicated }\end{array}$} \\
\cline { 2 - 4 } in years & $\mathbf{7}$ & $\mathbf{1 4}$ & $\mathbf{3 0}$ \\
\hline & & 4.7 & 6.2 \\
10 & 3.7 & 2.3 & 3.0 \\
\hline
\end{tabular}




\section{RÍO DESCALABRADO BASIN}

\section{Río Descalabrado near Sanja Blanca, Puerto Rico}

LOCATION.--Lat $18^{\circ} 05^{\prime} 24^{\prime \prime}$, long $66^{\circ} 24^{\prime} 30^{\prime \prime}$, Hydrologic Unit 21010004, at barrio Santa Catalina, on Highway $150,2.0 \mathrm{mi}(3.2 \mathrm{~km})$ southeast from Lago Toa Vaca, and $3.4 \mathrm{mi}(5.5 \mathrm{~km})$ northwest from Plaza de Coamo.

DRAINAGE AREA.--4.3 $\mathrm{mi}^{2}\left(11 \mathrm{~km}^{2}\right)$.

LOW-FLOW ESTIMATES.--Based on correlation of 8 base-flow measurements with concurrent base flows at gaging station 50112500 . MAGNITUDE AND FREQUENCY OF ANNUAL LOW FLOWS

\begin{tabular}{cccc}
\hline $\begin{array}{c}\text { Recurrence } \\
\text { interval, }\end{array}$ & \multicolumn{2}{c}{$\begin{array}{c}\text { Lowest average flow, in cubic feet per second, } \\
\text { for indicated number of consecutive days }\end{array}$} \\
\cline { 2 - 4 } in years & $\mathbf{7}$ & $\mathbf{1 4}$ & $\mathbf{3 0}$ \\
\hline 2 & .1 & .1 & .2 \\
10 & $<.1$ & $<.1$ & $<.1$ \\
\hline
\end{tabular}

50108200 Río Descalabrado at Las Ollas, Puerto Rico

LOCATION.--Lat $18^{\circ} 02^{\prime} 10^{\prime \prime}$, long 66 $25^{\prime} 36^{\prime \prime}$, Hydrologic Unit 2101004 , at barrio Descalabrado, on Highway $536,0.6 \mathrm{mi}(1.0 \mathrm{~km})$ upstream from Highway $52,2.2 \mathrm{mi}(3.5 \mathrm{~km})$ northwest from Cerro del Muerto.

DRAINAGE AREA.-- $14 \mathrm{mi}^{2}\left(36 \mathrm{~km}^{2}\right)$.

LOW-FLOW ESTIMATES.--Based on correlation of 8 base-flow measurements with concurrent base flows at gaging station 50112500 .

REMARKS.--Minor diversions are made above the station for public-water supply. Part of the flow infiltrates the aquifer.

MAGNITUDE AND FREQUENCY OF ANNUAL LOW FLOWS

\begin{tabular}{|c|c|c|c|}
\hline \multirow{2}{*}{$\begin{array}{c}\text { Recurrence } \\
\text { interval, } \\
\text { in years }\end{array}$} & \multicolumn{3}{|c|}{$\begin{array}{l}\text { Lowest average flow, in cubic feet per second } \\
\text { for indicated number of consecutive days }\end{array}$} \\
\hline & 7 & 14 & 30 \\
\hline $\begin{array}{r}2 \\
10\end{array}$ & $\begin{array}{l}<.1 \\
<.1\end{array}$ & $\begin{array}{r}.1 \\
<.1\end{array}$ & $\begin{array}{r}.2 \\
<.1\end{array}$ \\
\hline
\end{tabular}

RÍO CAÑAS BASIN

50109000 Río Cañas near Juana Díaz, Puerto Rico

LOCATION.--Lat $18^{\circ} 02^{\prime} 41^{\prime \prime}$, long $66^{\circ} 27^{\prime} 26^{\prime \prime}$, Hydrologic Unit 21010004, at barrio Cañas Arriba, on Highway 14 , and $3.3 \mathrm{mi}(5.3 \mathrm{~km})$ east from Plaza de Juana Díaz.

DRAINAGE AREA.--2.9 $\mathrm{mi}^{2}\left(7.5 \mathrm{~km}^{2}\right)$.

LOW-FLOW ESTIMATES.--Based on correlation of 8 base-flow measurements with concurrent base flows at gaging station 50112500 .

MAGNITUDE AND FREQUENCY OF ANNUAL LOW FLOWS

\begin{tabular}{cccc}
\hline $\begin{array}{c}\text { Recurrence } \\
\text { interval, } \\
\text { in years }\end{array}$ & \multicolumn{2}{c}{$\begin{array}{c}\text { Lowest average flow, in cubic feet per second, } \\
\text { for indicated number of consecutive days }\end{array}$} \\
\cline { 2 - 4 } & $\mathbf{7}$ & $\mathbf{1 4}$ & $\mathbf{3 0}$ \\
\hline 2 & $<.1$ & $<.1$ & $<.1$ \\
10 & $<.1$ & $<.1$ & $<.1$ \\
\hline
\end{tabular}

50109500 Río Cañas near Santa Isabel, Puerto Rico

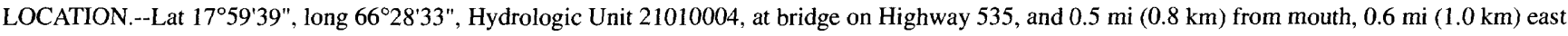
of Pastillo, and $5.1 \mathrm{mi}(8.2 \mathrm{~km})$ northwest from Plaza de Santa Isabel.

DRAINAGE AREA.--6.4 $\mathrm{mi}^{2}\left(16 \mathrm{~km}^{2}\right)$.

LOW-FLOW ESTIMATES.--Estimated minimum average 7-, 14-, and 30-day flow with 2- and 10-year recurrence interval is 0.0 on the basis of 7 observations of no flow under base-flow conditions. 


\section{Río Jacaguas at Villalba, Puerto Rico}

LOCATION.--Lat $18^{\circ} 07^{\prime} 37^{\prime \prime}$, long 66 $29^{\prime} 42^{\prime \prime}$, Hydrologic Unit 21010004 , at barrio Hato Puerco Arriba, upstream from the sewage water-treatment plant discharge point, $100 \mathrm{ft}(30 \mathrm{~m})$ downstream from confluence with Quebrada Achiote, and $0.2 \mathrm{mi}(0.3 \mathrm{~km})$ southwest from Villalba.

DRAINAGE AREA.-- $12 \mathrm{mi}^{2}\left(32 \mathrm{~km}^{2}\right)$

LOW-FLOW ESTIMATES.--Based on correlation of 8 base-flow measurements with concurrent base flows at gaging station 50112500 .

REMARKS.--Minor diversions are made above station to filter plant for public-water supply.

MAGNITUDE AND FREQUENCY OF ANNUAL LOW FLOWS

\begin{tabular}{cccc}
\hline $\begin{array}{c}\text { Recurrence } \\
\text { interval, } \\
\text { in years }\end{array}$ & \multicolumn{2}{c}{$\begin{array}{c}\text { Lowest average flow, in cubic feet per second, } \\
\text { for indicated number of consecutive days }\end{array}$} \\
\cline { 2 - 4 } & $\mathbf{7}$ & $\mathbf{1 4}$ & $\mathbf{3 0}$ \\
\hline 2 & 34 & 37 & 40 \\
10 & 28 & 30 & 32 \\
\hline
\end{tabular}

50110700 Río Toa Vaca at Pedro García, Puerto Rico

LOCATION.--Lat $18^{\circ} 08^{\prime} 11^{\prime \prime}$, long 66 $23^{\prime} 47^{\prime \prime}$, Hydrologic Unit 21010004, at barrio Pedro García, $2.1 \mathrm{mi}$ (3.4 km) southeast from intersection of Highways 143 and 155 , and $4.1 \mathrm{mi}(6.6 \mathrm{~km})$ northeast from Lago Toa Vaca.

DRAINAGE AREA.--3.1 $\mathrm{mi}^{2}\left(8.0 \mathrm{~km}^{2}\right)$.

LOW-FLOW ESTIMATES.--Based on correlation of 8 base-flow measurements with concurrent base flows at gaging station 50112500. MAGNITUDE AND FREQUENCY OF ANNUAL LOW FLOWS

\begin{tabular}{cccc}
\hline $\begin{array}{c}\text { Recurrence } \\
\text { interval, } \\
\text { in years }\end{array}$ & \multicolumn{2}{c}{$\begin{array}{c}\text { Lowest average flow, in cubic feet per second, } \\
\text { for indicated number of consecutive days }\end{array}$} \\
\cline { 2 - 4 } & $\mathbf{7}$ & $\mathbf{1 4}$ & $\mathbf{3 0}$ \\
\hline 2 & $<.1$ & .1 & .2 \\
10 & $<.1$ & $<.1$ & $<.1$ \\
\hline
\end{tabular}

\section{P 50110900 Río Toa Vaca upstream from Lago Toa Vaca, Puerto Rico}

LOCATION.--Lat $18^{\circ} 07^{\prime} 36^{\prime \prime}$, long $66^{\circ} 27^{\prime} 25^{\prime \prime}$, Hydrologic Unit 2101004, at barrio Caonillas Arriba, on Highway $553,0.5 \mathrm{mi}(0.8 \mathrm{~km})$ upstream from Lago Toa Vaca, and $2.4 \mathrm{mi}(3.9 \mathrm{~km})$ east from Villalba.

DRAINAGE AREA.--14 $\mathrm{mi}^{2}\left(37 \mathrm{~km}^{2}\right)$.

LOW-FLOW ESTIMATES.--Based on correlation of 8 base-flow measurements with concurrent base flows at gaging station 50112500.

MAGNITUDE AND FREQUENCY OF ANNUAL LOW FLOWS

\begin{tabular}{cccc}
\hline $\begin{array}{c}\text { Recurrence } \\
\text { interval, } \\
\text { in years }\end{array}$ & \multicolumn{2}{c}{$\begin{array}{c}\text { Lowest average flow, in cubic feet per second, } \\
\text { for indicated number of consecutive days }\end{array}$} \\
\cline { 2 - 4 } & $\mathbf{7}$ & $\mathbf{1 4}$ & $\mathbf{3 0}$ \\
\hline 2 & 1.1 & 1.4 & 1.9 \\
10 & .6 & .7 & 1.0 \\
\hline
\end{tabular}

50111720 Quebrada Guanábana near Juana Díaz, Puerto Rico

LOCATION.--Lat 1803'12", long 66²9'02", Hydrologic Unit 21010004, at barrio Tijeras, on Highway 14, 1.5 mi (2.4 km) east from Plaza de Juana Díaz. DRAINAGE AREA.--1.7 $\mathrm{mi}^{2}\left(4.5 \mathrm{~km}^{2}\right)$.

REMARKS.--Estimated minimum average 7-, 14-, and 30-day flow with 2- and 10-year recurrence interval is 0.0 on the basis of 6 observations of no flow under base-flow conditions. 


\section{RÍO INABÓN BASIN}

\section{Río Inabón at Real Anón, Puerto Rico}

LOCATION.-- $18^{\circ} 07^{\prime 2} 22^{\prime \prime}$, long 66³4'20", Hydrologic Unit 21010004, at barrio Anón, on Highway 511, $1.0 \mathrm{mi}$ (1.6 km) northeast from Cerro Santo Domingo, and $4.5 \mathrm{mi}(7.2 \mathrm{~km})$ northwest from Lago Guayabal.

DRAINAGE AREA.--6.0 $\mathrm{mi}^{2}\left(15 \mathrm{~km}^{2}\right)$.

LOW-FLOW ESTIMATES.--Based on correlation of 8 base-flow measurements with concurrent base flows at gaging station 50112500 .

REMARKS.--Minor diversions are made above station to filter plant for public-water supply.

MAGNITUDE AND FREQUENCY OF ANNUAL LOW FLOWS

\begin{tabular}{cccc}
\hline $\begin{array}{c}\text { Recurrence } \\
\text { interval, }\end{array}$ & \multicolumn{2}{c}{$\begin{array}{c}\text { Lowest average flow, in cubic feet per second, } \\
\text { for indicated number of consecutive days }\end{array}$} \\
\cline { 2 - 4 } in years & $\mathbf{7}$ & $\mathbf{1 4}$ & $\mathbf{3 0}$ \\
\hline 2 & 1.6 & 1.9 & 2.3 \\
10 & 1.0 & 1.2 & 1.4 \\
\hline
\end{tabular}

50112700 Río Guayo near Collores, Puerto Rico

LOCATION.--Lat $18^{\circ} 07^{\prime 2} 24^{\prime \prime}$, long 66 33'27", Hydrologic Unit 2101004, at barrio Collores, on Highway 517, about $400 \mathrm{ft}$ (122 m) west from Escuela Guaraguao, $0.9 \mathrm{mi}(1.4 \mathrm{~km})$ northwest from intersection of Highways 517 and 512, and $3.5 \mathrm{mi}(5.6 \mathrm{~km})$ northwest from Lago Toa Vaca.

DRAINAGE AREA.--1.7 $\mathrm{mi}^{2}\left(4.3 \mathrm{~km}^{2}\right)$.

LOW-FLOW ESTIMATES.--Based on correlation of 8 base-flow measurements with concurrent base flows at gaging station 50112500 . MAGNITUDE AND FREQUENCY OF ANNUAL LOW FLOWS

\begin{tabular}{cccc}
\hline $\begin{array}{c}\text { Recurrence } \\
\text { interval, }\end{array}$ & \multicolumn{2}{c}{$\begin{array}{c}\text { Lowest average flow, in cubic feet per second, } \\
\text { for indicated number of consecutive days }\end{array}$} \\
\cline { 2 - 4 } in years & $\mathbf{7}$ & $\mathbf{1 4}$ & $\mathbf{3 0}$ \\
\hline 2 & .5 & .6 & .7 \\
10 & .4 & .4 & .5 \\
\hline
\end{tabular}

50112750 Quebrada Indalecia at Collores, Puerto Rico

LOCATION.--Lat $18^{\circ} 06^{\prime} 33^{\prime \prime}$, long 66 $32^{\prime} 20^{\prime \prime}$, Hydrologic Unit 21010004, at barrio Collores, $200 \mathrm{ft}$ (61 m) upstream from confluence with Río Guayo, 0.9 $\mathrm{mi}(1.4 \mathrm{~km})$ northeast from Cerro Agustinillo, and $2.2 \mathrm{mi}(3.5 \mathrm{~km})$ northwest from Lago Guayabal.

DRAINAGE AREA.--3.5 $\mathrm{mi}^{2}\left(9.1 \mathrm{~km}^{2}\right)$.

LOW-FLOW ESTIMATES.--Estimated minimum average 7-, 14-, and 30-day flow with 2- and 10-year recurrence interval is 0.0 on the basis of 5 observations of no flow under base-flow conditions.

\section{Río Guayo upstream from Diversion at Collores, Puerto Rico}

LOCATION.--Lat $18^{\circ} 05^{\prime} 10^{\prime \prime}$, long 66 32'24", Hydrologic Unit 21010004, at barrio Collores, $2.1 \mathrm{mi}$ (3.4 km) southwest from Lago Guayabal, and $3.1 \mathrm{mi}$ $(5.0 \mathrm{~km})$ northwest from Plaza de Juana Díaz.

DRAINAGE AREA.--9.6 $\mathrm{mi}^{2}\left(25 \mathrm{~km}^{2}\right)$.

LOW-FLOW ESTIMATES.--Based on correlation of 8 base-flow measurements with concurrent base flows at gaging station 50112500 .

REMARKS.--Minor diversions are made above the station for public-water supply.

MAGNITUDE AND FREQUENCY OF ANNUAL LOW FLOWS

\begin{tabular}{cccc}
\hline $\begin{array}{c}\text { Recurrence } \\
\text { interval, } \\
\text { in years }\end{array}$ & \multicolumn{2}{c}{$\begin{array}{c}\text { Lowest average flow, in cubic feet per second, } \\
\text { for indicated number of consecutive days }\end{array}$} \\
\cline { 2 - 4 } & $\mathbf{7}$ & $\mathbf{1 4}$ & $\mathbf{3 0}$ \\
\hline 2 & .8 & 1.0 & 1.3 \\
10 & .4 & .5 & .7 \\
\hline
\end{tabular}




\section{RÍO BUCANÁ BASIN}

\section{Río San Patricio upstream from Lago Cerrillos, Puerto Rico}

LOCATION.--Lat $18^{\circ} 07^{\prime} 12^{\prime \prime}$, long 66³6'27, Hydrologic Unit 21010004, at barrio Maragüez, $1.5 \mathrm{mi}$ (2.4 km) northwest from Cerro Santo Domingo, $3.6 \mathrm{mi}$ $(5.8 \mathrm{~km})$ northwest from Lago Cerrillos, and $7.3 \mathrm{mi}(12 \mathrm{~km})$ from Plaza Degetau, Ponce.

DRAINAGE AREA.--5.8 $\mathrm{mi}^{2}\left(15 \mathrm{~km}^{2}\right)$.

LOW-FLOW ESTIMATES.--Based on correlation of 6 base-flow measurements with concurrent base flows at gaging station 50114000 .

MAGNITUDE AND FREQUENCY OF ANNUAL LOW FLOWS

\begin{tabular}{cccc}
\hline $\begin{array}{c}\text { Recurrence } \\
\text { interval, }\end{array}$ & \multicolumn{2}{c}{$\begin{array}{c}\text { Lowest average flow, in cubic feet per second, } \\
\text { for indicated number of consecutive days }\end{array}$} \\
\cline { 2 - 4 } in years & $\mathbf{7}$ & $\mathbf{1 4}$ & $\mathbf{3 0}$ \\
\hline 2 & 2.5 & 2.6 & 3.1 \\
10 & 1.5 & 1.9 & 2.3 \\
\hline
\end{tabular}

50113800 Río Cerrillos upstream from Lago Cerrillos, Puerto Rico

LOCATION.--Lat $18^{\circ} 07^{\prime} 01^{\prime \prime}$, long 66³6'17", Hydrologic Unit 21010004, at barrio Maragüez, $1.3 \mathrm{mi}(2.1 \mathrm{~km})$ west from Cerro Santo Domingo, 3.3 mi (5.3 $\mathrm{km})$ northwest from Lago Cerrillos, and $7.2 \mathrm{mi}(12 \mathrm{~km})$ from Plaza Degetau, Ponce.

DRAINAGE AREA.-- $12 \mathrm{mi}^{2}\left(31 \mathrm{~km}^{2}\right)$.

LOW-FLOW ESTIMATES.--Based on correlation of 20 base-flow measurements with concurrent base flows at gaging station 50114000.

$$
\text { MAGNITUDE AND FREQUENCY OF ANNUAL LOW FLOWS }
$$

\begin{tabular}{cccc}
\hline $\begin{array}{c}\text { Recurrence } \\
\text { interval, }\end{array}$ & \multicolumn{2}{c}{$\begin{array}{c}\text { Lowest average flow, in cubic feet per second, } \\
\text { for indicated number of consecutive days }\end{array}$} \\
\cline { 2 - 4 } in years & $\mathbf{7}$ & $\mathbf{1 4}$ & $\mathbf{3 0}$ \\
\hline 2 & 4.0 & 4.4 & 5.4 \\
10 & 2.5 & 3.0 & 3.7 \\
\hline
\end{tabular}

50114150 Quebrada Ausubo near Ponce, Puerto Rico

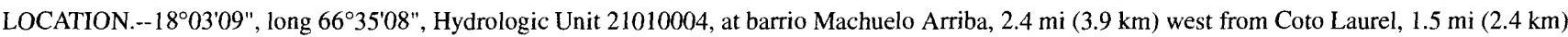
south from Lago Cerrillos, and $3.8 \mathrm{mi}(6.1 \mathrm{~km})$ northeast from Plaza Degetau, Ponce.

DRAINAGE AREA.-- $1.2 \mathrm{mi}^{2}\left(3.0 \mathrm{~km}^{2}\right)$.

REMARKS.--Estimated minimum average 7-, 14-, and 30-day flow with 2- and 10-year recurrence interval is 0.0 on the basis of 8 observations of no flow under base-flow conditions.

\section{Río Bayagán near Ponce, Puerto Rico}

LOCATION.--Lat $18^{\circ} 02^{\prime} 51^{\prime \prime}$, long $66^{\circ} 35^{\prime} 12^{\prime \prime}$, Hydrologic Unit 21010004, at barrio Machuelo Arriba, $2.5 \mathrm{mi}$ (4.0 km) west of Coto Laurel, $1.9 \mathrm{mi}(3.0 \mathrm{~km})$ south from Lago Cerrillos, and $3.0 \mathrm{mi}(4.8 \mathrm{~km})$ northeast from Plaza Degetau, Ponce.

DRAINAGE AREA.--3.8 $\mathrm{mi}^{2}\left(9.9 \mathrm{~km}^{2}\right)$.

REMARKS.--Estimated minimum average 7-, 14-, and 30-day flow with 2- and 10-year recurrence interval is 0.0 on the basis of 8 observations of no flow under base-flow conditions. 


\section{RÍO PORTUGUÉS BASIN}

\section{Río Portugués near Tibes, Puerto Rico}

LOCATION.--Lat $18^{\circ} 04^{\prime 26}$ ", long 66³8'35", Hydrologic Unit 21010004, at barrio Tibes, $0.5 \mathrm{mi}(0.8 \mathrm{~km})$ southwest from Cerro del Diablo, $6.0 \mathrm{mi}(9.6 \mathrm{~km})$ northeast from Peñuelas, and $6.2 \mathrm{mi}(10 \mathrm{~km})$ north from Ponce.

DRAINAGE AREA.--7.3 $\mathrm{mi}^{2}\left(19 \mathrm{~km}^{2}\right)$.

LOW-FLOW ESTIMATES.--Based on correlation of 8 base-flow measurements with concurrent base flows at gaging station 50115000.

$$
\text { MAGNITUDE AND FREQUENCY OF ANNUAL LOW FLOWS }
$$

\begin{tabular}{cccc}
\hline $\begin{array}{c}\text { Recurrence } \\
\text { interval, } \\
\text { in years }\end{array}$ & \multicolumn{2}{c}{$\begin{array}{c}\text { Lowest average flow, in cubic feet per second, } \\
\text { for indicated number of consecutive days }\end{array}$} \\
\cline { 2 - 4 } & $\mathbf{7}$ & $\mathbf{1 4}$ & $\mathbf{3 0}$ \\
\hline 2 & 2.4 & 2.6 & 3.0 \\
10 & 1.6 & 1.8 & 2.0 \\
\hline
\end{tabular}

50115400 Río Portugués near Ponce, Puerto Rico

LOCATION.--Lat $18^{\circ} 02^{\prime 2} 7^{\prime \prime}$, long $66^{\circ} 36^{\prime} 41^{\prime \prime}$, Hydrologic Unit 21010004 , at barrio Portugués, $1.0 \mathrm{mi}(1.6 \mathrm{~km})$ west from Jardines de Ponce, $0.4 \mathrm{mi}(0.6 \mathrm{~km})$ north from confluence with Río Chiquito, and $1.9 \mathrm{mi}(3.0 \mathrm{~km})$ north from Plaza Degetau, Ponce.

DRAINAGE AREA.--12 $\mathrm{mi}^{2}\left(32 \mathrm{~km}^{2}\right)$.

LOW-FLOW ESTIMATES.--Based on correlation of 8 base-flow measurements with concurrent base flows at gaging station 50115000 .

REMARKS.--Minor diversions are made above the station for public-water supply.

MAGNITUDE AND FREQUENCY OF ANNUAL LOW FLOWS

\begin{tabular}{cccc}
\hline $\begin{array}{c}\text { Recurrence } \\
\text { interval, }\end{array}$ & \multicolumn{2}{c}{$\begin{array}{c}\text { Lowest average flow, in cubic feet per second, } \\
\text { for indicated number of consecutive days }\end{array}$} \\
\cline { 2 - 4 } in years & $\mathbf{7}$ & $\mathbf{1 4}$ & $\mathbf{3 0}$ \\
\hline 2 & 1.5 & 1.8 & 2.2 \\
10 & .9 & 1.0 & 1.2 \\
\hline
\end{tabular}

50115450 Río Chiquito at Portugués, Puerto Rico

LOCATION.--Lat $18^{\circ} 04^{\prime} 11^{\prime \prime}$, long 66³7'00", Hydrologic Unit 21010004, at barrio Portugués, $2.1 \mathrm{mi}(3.4 \mathrm{~km})$ northwest from Jardines de Ponce, 1.7 mi (2.7 $\mathrm{km})$ southwest from Pico Pinto, and $2.8 \mathrm{mi}(4.5 \mathrm{~km})$ north from Plaza Degetau, Ponce.

DRAINAGE AREA.--3.1 $\mathrm{mi}^{2}\left(8.1 \mathrm{~km}^{2}\right)$.

LOW-FLOW ESTIMATES.--Based on correlation of 8 base-flow measurements with concurrent base flows at gaging station 50115000.

MAGNITUDE AND FREQUENCY OF ANNUAL LOW FLOWS

\begin{tabular}{cccc}
\hline $\begin{array}{c}\text { Recurrence } \\
\text { interval, } \\
\text { in years }\end{array}$ & \multicolumn{2}{c}{$\begin{array}{c}\text { Lowest average flow, in cubic feet per second, } \\
\text { for indicated number of consecutive days }\end{array}$} \\
\cline { 2 - 4 } & $\mathbf{7}$ & $\mathbf{1 4}$ & $\mathbf{3 0}$ \\
\hline 2 & .2 & .2 & .3 \\
10 & $<.1$ & .1 & .1 \\
\hline
\end{tabular}




\section{RÍO PORTUGUÉS BASIN—Continued \\ 50115600 Río Chiquito near Ponce, Puerto Rico}

LOCATION.--Lat $18^{\circ} 02^{\prime} 37^{\prime \prime}$, long 66³6'31", Hydrologic Unit 21010004, at barrio Portugués, $0.6 \mathrm{mi}(1.0 \mathrm{~km})$ west from Jardines de Ponce, $0.8 \mathrm{mi}(1.3 \mathrm{~km})$ south from Cerro El Gato, and $2.1 \mathrm{mi}(3.4 \mathrm{~km})$ north from Plaza Degetau, Ponce.

DRAINAGE AREA.-- $4.4 \mathrm{mi}^{2}\left(12 \mathrm{~km}^{2}\right)$.

LOW-FLOW ESTIMATES.--Based on correlation of 8 base-flow measurements with concurrent base flows at gaging station 50115000

MAGNITUDE AND FREQUENCY OF ANNUAL LOW FLOWS

\begin{tabular}{cccc}
\hline $\begin{array}{c}\text { Recurrence } \\
\text { interval, } \\
\text { in years }\end{array}$ & \multicolumn{2}{c}{$\begin{array}{c}\text { Lowest average flow, in cubic feet per second, } \\
\text { for indicated number of consecutive days }\end{array}$} \\
\cline { 2 - 4 } & $\mathbf{7}$ & $\mathbf{1 4}$ & $\mathbf{3 0}$ \\
\hline 2 & $<.1$ & .1 & .1 \\
10 & $<.1$ & $<.1$ & $<.1$ \\
\hline
\end{tabular}

\section{Río Portugués at Highway 2 By-Pass at Ponce, Puerto Rico}

LOCATION.--Lat $17^{\circ} 59^{\prime} 52^{\prime \prime}$, long 66 $36^{\circ} 52^{\prime \prime}$, Hydrologic Unit 21010004, at bridge on Highway 2 By-Pass, 2.0 mi (3.2 km) upstream from mouth, and 1.1 mi $(1.8 \mathrm{~km})$ south from Plaza Degetau, Ponce.

DRAINAGE AREA.--20 $\mathrm{mi}^{2}\left(53 \mathrm{~km}^{2}\right)$.

LOW-FLOW ESTIMATES.--Based on correlation of 8 base-flow measurements with concurrent base flows at gaging station 50115000.

REMARKS.--Minor diversions are made above the station for public-water supply.

MAGNITUDE AND FREQUENCY OF ANNUAL LOW FLOWS

\begin{tabular}{cccc}
\hline $\begin{array}{c}\text { Recurrence } \\
\text { interval, } \\
\text { in years }\end{array}$ & \multicolumn{2}{c}{$\begin{array}{c}\text { Lowest average flow, in cubic feet per second, } \\
\text { for indicated number of consecutive days }\end{array}$} \\
\hline & $\mathbf{7}$ & $\mathbf{1 4}$ & $\mathbf{3 0}$ \\
\hline 2 & .6 & .7 & .9 \\
10 & .2 & .3 & .4 \\
\hline
\end{tabular}

RÍO MATILDE BASIN

50116800 Río Cañas at Magueyes, Puerto Rico

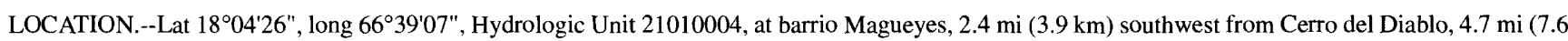
$\mathrm{km})$ northwest from Peñuelas, and $4.0 \mathrm{mi}(6.4 \mathrm{~km})$ northwest from Ponce.

DRAINAGE AREA. $-4.0 \mathrm{mi}^{2}\left(10 \mathrm{~km}^{2}\right)$.

LOW-FLOW ESTIMATES.--Based on correlation of 8 base-flow measurements with concurrent base flows at gaging station 50115000 .

MAGNITUDE AND FREQUENCY OF ANNUAL LOW FLOWS

\begin{tabular}{cccc}
\hline $\begin{array}{c}\text { Recurrence } \\
\text { interval, } \\
\text { in years }\end{array}$ & \multicolumn{2}{c}{$\begin{array}{c}\text { Lowest average flow, in cubic feet per second, } \\
\text { for indicated number of consecutive days }\end{array}$} \\
\hline & $\mathbf{7}$ & $\mathbf{1 4}$ & $\mathbf{3 0}$ \\
\hline 2 & 1.6 & 1.8 & 2.0 \\
10 & 1.2 & 1.3 & 1.4 \\
\hline
\end{tabular}




\section{RÍO MATILDE BASIN-Continued}

\section{Río Cañas downstream from Las Américas Ave., Puerto Rico}

LOCATION.--Lat 1800'37", long 66³8'26", Hydrologic Unit 21010004, $0.5 \mathrm{mi}(0.8 \mathrm{~km})$ upstream from confluence with Río Pastillo, and $1.1 \mathrm{mi}(1.7 \mathrm{~km})$ west from Escuela Dr. Pila, PR.

DRAINAGE AREA.--8.5 $\mathrm{mi}^{2}\left(22 \mathrm{~km}^{2}\right)$.

LOW-FLOW ESTIMATES.--Based on correlation of 8 base-flow measurements with concurrent base flows at gaging station 50115000 .

REMARKS.--Minor diversions are made above the station for public-water supply.

MAGNITUDE AND FREQUENCY OF ANNUAL LOW FLOWS

\begin{tabular}{cccc}
\hline $\begin{array}{c}\text { Recurrence } \\
\text { interval, }\end{array}$ & \multicolumn{2}{c}{$\begin{array}{c}\text { Lowest average flow, in cubic feet per second, } \\
\text { for indicated number of consecutive days }\end{array}$} \\
\cline { 2 - 3 } in years & $\mathbf{7}$ & $\mathbf{1 4}$ & $\mathbf{3 0}$ \\
\hline 2 & 4.2 & 4.4 & 5.0 \\
10 & 3.0 & 3.2 & 3.6 \\
\hline
\end{tabular}

50117800 Río Pastillo at Pastillo, Puerto Rico

LOCATION.--Lat $18^{\circ} 02^{\prime} 53^{\prime \prime}$, long $66^{\circ} 39^{\prime} 52^{\prime \prime}$, Hydrologic Unit 21010004, at barrio Quebrada Limón on Highway $502,0.8 \mathrm{mi}(1.3 \mathrm{~km})$ northwest of intersection of Highways 502 and 132, $0.9 \mathrm{mi}(1.4 \mathrm{~km})$ west of Magueyes, and $3.1 \mathrm{mi}(5.0 \mathrm{~km})$ northwest from Ponce.

DRAINAGE AREA.-- $4.3 \mathrm{mi}^{2}\left(11 \mathrm{~km}^{2}\right)$.

LOW-FLOW ESTIMATES.--Based on correlation of 8 base-flow measurements with concurrent base flows at gaging station 50115000 .

MAGNITUDE AND FREQUENCY OF ANNUAL LOW FLOWS

\begin{tabular}{cccc}
\hline $\begin{array}{c}\text { Recurrence } \\
\text { interval, } \\
\text { in years }\end{array}$ & $\begin{array}{c}\text { Lowest average flow, in cubic feet per second, } \\
\text { for indicated number of consecutive days }\end{array}$ \\
\hline 2 & $\mathbf{7}$ & $\mathbf{1 4}$ & $\mathbf{3 0}$ \\
10 & .1 & .1 & .2 \\
& $<.1$ & $<.1$ \\
\hline
\end{tabular}

50118300 Río Pastillo near Ponce, Puerto Rico

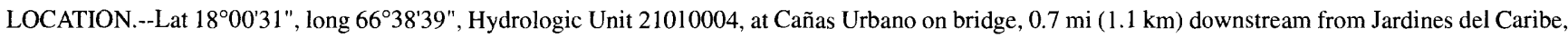
and $1.1 \mathrm{mi}(1.7 \mathrm{~km})$ west of Escuela Dr. Pila, Ponce.

DRAINAGE AREA.--11 $\mathrm{mi}^{2}\left(28 \mathrm{~km}^{2}\right)$.

REMARKS.--Estimated minimum average 7-, 14-, and 30-day flow with 2- and 10-year recurrence interval is 0.0 on the basis of 6 observations of no flow under base-flow conditions.

\section{Río Matilde at Ponce, Puerto Rico}

LOCATION.--Lat 1759'53", long 66³8'06", Hydrologic Unit 21010004, at Highway 2, $1.1 \mathrm{mi}(1.8 \mathrm{~km})$ upstream from mouth.

DRAINAGE AREA.--20 $\mathrm{mi}^{2}\left(53 \mathrm{~km}^{2}\right)$.

LOW-FLOW ESTIMATES.--Based on correlation of 8 base-flow measurements with concurrent base flows at gaging station 50115000 .

REMARKS.--Minor diversions are made above the station for public-water supply.

MAGNITUDE AND FREQUENCY OF ANNUAL LOW FLOWS

\begin{tabular}{cccc}
\hline $\begin{array}{c}\text { Recurrence } \\
\text { interval, }\end{array}$ & \multicolumn{2}{c}{$\begin{array}{c}\text { Lowest average flow, in cubic feet per second, } \\
\text { for indicated number of consecutive days }\end{array}$} \\
\cline { 2 - 4 } in years & $\mathbf{7}$ & $\mathbf{1 4}$ & $\mathbf{3 0}$ \\
\hline 2 & 3.2 & 3.6 & 4.3 \\
10 & 1.9 & 2.1 & 2.5 \\
\hline
\end{tabular}




\section{RÍO MATILDE BASIN-Continued}

\section{Quebrada del Agua at Playa de Ponce, Puerto Rico}

LOCATION.--Lat 1759'13", long 66³8'22", Hydrologic Unit 21010004, $700 \mathrm{ft}$ (213 m) upstream from confluence with Río Matilde.

DRAINAGE AREA.--6.4 $\mathrm{mi}^{2}\left(17 \mathrm{~km}^{2}\right)$.

REMARKS.--Estimated minimum average 7-, 14-, and 30-day flow with 2 and 10-day recurrence interval is 0.0 on the basis of 8 observations of no flow under base-flow conditions.

\section{RÍO TALLABOA BASIN}

\section{Río Tallaboa near Quebrada Ceiba, Puerto Rico}

LOCATION.--Lat $18^{\circ} 04^{\prime} 18^{\prime \prime}$, long 66 $42^{\prime} 03^{\prime \prime}$, Hydrologic Unit 21010004, at barrio Quebrada Ceiba, $0.06 \mathrm{mi}(0.1 \mathrm{~km})$ west from Highway $391,1.2 \mathrm{mi}(2.0$ $\mathrm{km})$ north from Tallaboa Alta, and $1.7 \mathrm{mi}(2.7 \mathrm{~km})$ northeast from Plaza de Peñuelas.

DRAINAGE AREA. $-8.4 \mathrm{mi}^{2}\left(22 \mathrm{~km}^{2}\right)$

LOW-FLOW ESTIMATES.--Based on correlation of 8 base-flow measurements with concurrent base flows at gaging station 50124200 .

\section{MAGNITUDE AND FREQUENCY OF ANNUAL LOW FLOWS}

\begin{tabular}{cccc}
\hline $\begin{array}{c}\text { Recurrence } \\
\text { interval, }\end{array}$ & \multicolumn{2}{c}{$\begin{array}{c}\text { Lowest average flow, in cubic feet per second, } \\
\text { for indicated number of consecutive days }\end{array}$} \\
\cline { 2 - 4 } in years & $\mathbf{7}$ & $\mathbf{1 4}$ & $\mathbf{3 0}$ \\
\hline 2 & 3.5 & 3.7 & 4.2 \\
10 & 2.9 & 3.4 & 3.6 \\
\hline
\end{tabular}

50120700 Río Guayanés near Peñuelas, Puerto Rico

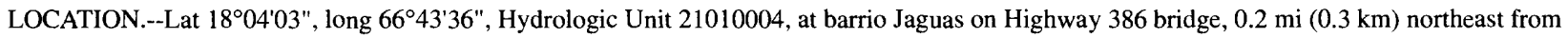
intersection of Highways 386 and 132, $0.6 \mathrm{mi}(1.0 \mathrm{~km})$ northeast from Plaza de Peñuelas.

DRAINAGE AREA.--7.3 $\mathrm{mi}^{2}(19 \mathrm{~km})$.

LOW-FLOW ESTIMATES.--Based on correlation of 7 base-flow measurements with concurrent base flows at gaging station 50124200 .

REMARKS.--Flow is regulated by the hydroelectric plant.

MAGNITUDE AND FREQUENCY OF ANNUAL LOW FLOWS

\begin{tabular}{cccc}
\hline $\begin{array}{c}\text { Recurrence } \\
\text { interval, }\end{array}$ & \multicolumn{2}{c}{$\begin{array}{c}\text { Lowest average flow, in cubic feet per second, } \\
\text { for indicated number of consecutive days }\end{array}$} \\
\cline { 2 - 4 } in years & $\mathbf{7}$ & $\mathbf{1 4}$ & $\mathbf{3 0}$ \\
\hline 2 & 1.3 & 1.5 & 1.7 \\
10 & 1.0 & 1.2 & 1.4 \\
\hline
\end{tabular}

50121000 Río Tallaboa at Peñuelas, Puerto Rico

LOCATION.--Lat $18^{\circ} 03^{\prime} 02^{\prime \prime}$, long 66 $43^{\circ} 19^{\prime \prime}$, Hydrologic Unit 21010004, $350 \mathrm{ft}(107 \mathrm{~m})$ downstream from Highway $132 \mathrm{bridge}, 0.6 \mathrm{mi}(1.0 \mathrm{~km})$ south of Peñuelas.

DRAINAGE AREA.--24 $\mathrm{mi}^{2}\left(63 \mathrm{~km}^{2}\right)$.

LOW-FLOW ESTIMATES.--Based on correlation of 8 base-flow measurements with concurrent base flows at gaging station 50124200 .

REMARKS.--Flow is regulated by the hydroelectric plant.

MAGNITUDE AND FREQUENCY OF ANNUAL LOW FLOWS

\begin{tabular}{cccc}
\hline $\begin{array}{c}\text { Recurrence } \\
\text { interval, } \\
\text { in years }\end{array}$ & $\begin{array}{c}\text { Lowest average flow, in cubic feet per second, } \\
\text { for indicated number of consecutive days }\end{array}$ \\
\cline { 2 - 4 } 2 & $\mathbf{7}$ & $\mathbf{1 4}$ & $\mathbf{3 0}$ \\
\hline 10 & 6.0 & 6.6 & 7.6 \\
\hline
\end{tabular}




\section{Río Tallaboa at Tallaboa, Puerto Rico}

LOCATION.--Lat $18^{\circ} 00^{\prime} 31^{\prime \prime}$, long 66³'49", Hydrologic Unit 21010004, on bridge at Hacienda Dolores, $700 \mathrm{ft}(213 \mathrm{~m})$ upstream from Highway $127,0.8$ $\mathrm{mi}(1.3 \mathrm{~km})$ northwest of Tallaboa, and $7.6 \mathrm{mi}(12 \mathrm{~km})$ west of Plaza Degetau, Ponce.

DRAINAGE AREA.--32 $\mathrm{mi}^{2}\left(82 \mathrm{~km}^{2}\right)$.

LOW-FLOW ESTIMATES.--Based on correlation of 8 base-flow measurements with concurrent base flows at gaging station 50124200 .

REMARKS.--Flow is regulated by the hydroelectric plant.

MAGNITUDE AND FREQUENCY OF ANNUAL LOW FLOWS

\begin{tabular}{cccc}
\hline $\begin{array}{c}\text { Recurrence } \\
\text { interval, }\end{array}$ & \multicolumn{2}{c}{$\begin{array}{c}\text { Lowest average flow, in cubic feet per second, } \\
\text { for indicated number of consecutive days }\end{array}$} \\
\cline { 2 - 4 } in years & $\mathbf{7}$ & $\mathbf{1 4}$ & $\mathbf{3 0}$ \\
\hline 2 & 6.0 & 6.6 & 7.6 \\
10 & 4.8 & 5.6 & 6.3 \\
\hline
\end{tabular}

RÍO MACANÁ BASIN

50122500 Río Macaná near Peñuelas, Puerto Rico

LOCATION.--Lat $18^{\circ} 03^{\prime} 40^{\prime \prime}$, long $66^{\circ} 46^{\prime} 12^{\prime \prime}$, Hydrologic Unit 21010004 , at barrio Macaná at intersection of Highways 131 and $132,5.5 \mathrm{mi}(8.8 \mathrm{~km})$ northeast from Yauco, and $2.8 \mathrm{mi}(4.5 \mathrm{~km})$ northeast from Plaza de Guayanilla.

DRAINAGE AREA.-- $2.8 \mathrm{mi}^{2}\left(7.2 \mathrm{~km}^{2}\right)$.

LOW-FLOW ESTIMATES.--Based on correlation of 8 base-flow measurements with concurrent base flows at gaging station 50124200 .

REMARKS.--Flow is regulated by the hydroelectric plant.

MAGNITUDE AND FREQUENCY OF ANNUAL LOW FLOWS

\begin{tabular}{cccc}
\hline $\begin{array}{c}\text { Recurrence } \\
\text { interval, }\end{array}$ & \multicolumn{2}{c}{$\begin{array}{c}\text { Lowest average flow, in cubic feet per second, } \\
\text { for indicated number of consecutive days }\end{array}$} \\
\cline { 2 - 4 } in years & $\mathbf{7}$ & $\mathbf{1 4}$ & $\mathbf{3 0}$ \\
\hline 2 & .4 & .4 & .5 \\
10 & .3 & .3 & .4 \\
\hline
\end{tabular}

50122900 Río Macaná at Magas Arriba, Puerto Rico

LOCATION.--Lat $18^{\circ} 01^{\prime} 00^{\prime \prime}$, long 66 $45^{\prime} 57^{\prime \prime}$, Hydrologic Unit 21010004, $200 \mathrm{ft}(60 \mathrm{~m})$ upstream from bridge on Highway 2, $0.6 \mathrm{mi}(1.0 \mathrm{~km})$ upstream from mouth, and $1.8 \mathrm{mi}(2.8 \mathrm{~km})$ east of Plaza de Guayanilla.

DRAINAGE AREA.--9.0 $\mathrm{mi}^{2}\left(23 \mathrm{~km}^{2}\right)$.

REMARKS.--Estimated minimum average 7-, 14-, and 30-day flow with 2- and 10-year recurrence interval is 0.0 on the basis of 8 observations of no flow under base-flow conditions. 


\section{RÍO GUAYANILLA BASIN}

\section{Río Guayanilla at Pasto, Puerto Rico}

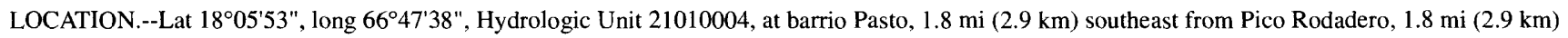
west from Cerro El Peligro, and $5.2 \mathrm{mi}(8.4 \mathrm{~km})$ north from Plaza de Guayanilla.

DRAINAGE AREA.--6.4 $\mathrm{mi}^{2}\left(17 \mathrm{~km}^{2}\right)$.

LOW-FLOW ESTIMATES.--Based on correlation of 8 base-flow measurements with concurrent base flows at gaging station 50124200.

REMARKS.--Minor diversions are made above the station for public-water supply.

MAGNITUDE AND FREQUENCY OF ANNUAL LOW FLOWS

\begin{tabular}{cccc}
\hline $\begin{array}{c}\text { Recurrence } \\
\text { interval, } \\
\text { in years }\end{array}$ & \multicolumn{2}{c}{$\begin{array}{c}\text { Lowest average flow, in cubic feet per second, } \\
\text { for indicated number of consecutive days }\end{array}$} \\
\cline { 2 - 4 } 2 & $\mathbf{7}$ & $\mathbf{1 4}$ & $\mathbf{3 0}$ \\
\hline 10 & 2.9 & 3.2 & 3.4 \\
\hline
\end{tabular}

50124600 Río Guayanilla near Central Rufina, Puerto Rico

LOCATION.--Lat $18^{\circ} 01^{\prime} 00^{\prime \prime}$, long $66^{\circ} 47^{\prime} 01^{\prime \prime}$, Hydrologic Unit 21010004 , at Guayanilla, $1.2 \mathrm{mi}(1.9 \mathrm{~km})$ upstream from mouth, $0.8 \mathrm{mi}(1.3 \mathrm{~km})$ northeast from Central Rufina, and $0.6 \mathrm{mi}(1.0 \mathrm{~km})$ southeast from Plaza de Guayanilla.

DRAINAGE AREA.--23 $\mathrm{mi}^{2}\left(60 \mathrm{~km}^{2}\right)$.

LOW-FLOW ESTIMATES.--Based on correlation of 8 base-flow measurements with concurrent base flows at gaging station 50124200.

REMARKS.--Minor diversions are made above the station to filter plant for public-water supply.

MAGNITUDE AND FREQUENCY OF ANNUAL LOW FLOWS

\begin{tabular}{cccc}
\hline $\begin{array}{c}\text { Recurrence } \\
\text { interval, } \\
\text { in years }\end{array}$ & \multicolumn{2}{c}{$\begin{array}{c}\text { Lowest average flow, in cubic feet per second, } \\
\text { for indicated number of consecutive days }\end{array}$} \\
\cline { 2 - 4 } 2 & 7 & $\mathbf{1 4}$ & $\mathbf{3 0}$ \\
\hline 10 & .2 & .3 & .5 \\
.2 & .3 \\
\hline
\end{tabular}

\section{Río Yauco near Lago Lucchetti Damsite, Puerto Rico}

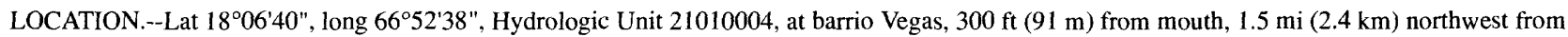
spillway, and $5.4 \mathrm{mi}(8.7 \mathrm{~km})$ northwest from Plaza de Yauco.

DRAINAGE AREA.--8.0 $\mathrm{mi}^{2}\left(21 \mathrm{~km}^{2}\right)$.

LOW-FLOW ESTIMATES.--Based on correlation of 8 base-flow measurements with concurrent base flows at gaging station 50136000.

MAGNITUDE AND FREQUENCY OF ANNUAL LOW FLOWS

\begin{tabular}{cccc}
\hline $\begin{array}{c}\text { Recurrence } \\
\text { interval, }\end{array}$ & \multicolumn{2}{c}{$\begin{array}{c}\text { Lowest average flow, in cubic feet per second, } \\
\text { for indicated number of consecutive days }\end{array}$} \\
\cline { 2 - 4 } in years & $\mathbf{7}$ & $\mathbf{1 4}$ & $\mathbf{3 0}$ \\
\hline 2 & 2.8 & 3.0 & 3.2 \\
10 & 1.2 & 1.3 & 1.7 \\
\hline
\end{tabular}




\section{RÍO YAUCO BASIN-Continued}

\section{Río Naranjo near Lago Lucchetti Damsite, Puerto Rico}

LOCATION.--Lat $18^{\circ} 06^{\prime} 20^{\prime \prime}$, long 66 $51^{\circ} 37^{\prime \prime}$, Hydrologic Unit 21010004, at barrio Naranjo on Highway $128,0.3 \mathrm{mi}(0.5 \mathrm{~km})$ from mouth, and $0.9 \mathrm{mi}(1.4$ $\mathrm{km})$ from spillway.

DRAINAGE AREA.--1.9 $\mathrm{mi}^{2}\left(5.0 \mathrm{~km}^{2}\right)$.

LOW-FLOW ESTIMATES.--Based on correlation of 8 base-flow measurements with concurrent base flows at gaging station 50124200 . MAGNITUDE AND FREQUENCY OF ANNUAL LOW FLOWS

\begin{tabular}{cccc}
\hline $\begin{array}{c}\text { Recurrence } \\
\text { interval, } \\
\text { in years }\end{array}$ & \multicolumn{2}{c}{$\begin{array}{c}\text { Lowest average flow, in cubic feet per second, } \\
\text { for indicated number of consecutive days }\end{array}$} \\
\cline { 2 - 4 } 2 & $\mathbf{7}$ & $\mathbf{1 4}$ & $\mathbf{3 0}$ \\
\hline 10 & .2 & .2 & .3 \\
\hline
\end{tabular}

\section{Quebrada Grande near Lago Lucchetti Damsite, Puerto Rico}

LOCATION.--Lat $18^{\circ} 06^{\prime} 20^{\prime \prime}$, long 66 50'56", Hydrologic Unit 21010004, at barrio Naranjo, $0.6 \mathrm{mi}(1.0 \mathrm{~km})$ west from Hacienda Roig, $0.9 \mathrm{mi}(1.4 \mathrm{~km})$ from mouth, and $1.3 \mathrm{mi}(2.1 \mathrm{~km})$ from spillway.

DRAINAGE AREA.--2.8 $\mathrm{mi}^{2}\left(7.3 \mathrm{~km}^{2}\right)$.

LOW-FLOW ESTIMATES.--Based on correlation of 8 base-flow measurements with concurrent base flows at gaging station 50124200 .

MAGNITUDE AND FREQUENCY OF ANNUAL LOW FLOWS

\begin{tabular}{cccc}
\hline $\begin{array}{c}\text { Recurrence } \\
\text { interval, }\end{array}$ & \multicolumn{2}{c}{$\begin{array}{c}\text { Lowest average flow, in cubic feet per second, } \\
\text { for indicated number of consecutive days }\end{array}$} \\
\cline { 2 - 4 } in years & $\mathbf{7}$ & $\mathbf{1 4}$ & $\mathbf{3 0}$ \\
\hline 2 & .6 & .7 & .8 \\
10 & .5 & .6 & .7 \\
\hline
\end{tabular}

50125860 Río Duey at Duey, Puerto Rico

LOCATION.--Lat $18^{\circ} 05^{\prime} 44^{\prime \prime}$, long 66 50'06", Hydrologic Unit 21010004, at Barrio Duey, $0.8 \mathrm{mi}(1.3 \mathrm{~km})$ southeast from Hacienda Roig, $1.2 \mathrm{mi}(1.9 \mathrm{~km})$ east from Lago Lucchetti, and $4.1 \mathrm{mi}(6.6 \mathrm{~km})$ from Plaza de Yauco.

DRAINAGE AREA.--4.6 $\mathrm{mi}^{2}\left(12 \mathrm{~km}^{2}\right)$.

LOW-FLOW ESTIMATES.--Based on correlation of 8 base-flow measurements with concurrent base flows at gaging station 50124200.

MAGNITUDE AND FREQUENCY OF ANNUAL LOW FLOWS

\begin{tabular}{cccc}
\hline $\begin{array}{c}\text { Recurrence } \\
\text { interval, }\end{array}$ & \multicolumn{2}{c}{$\begin{array}{c}\text { Lowest average flow, in cubic feet per second, } \\
\text { for indicated number of consecutive days }\end{array}$} \\
\cline { 2 - 4 } in years & $\mathbf{7}$ & $\mathbf{1 4}$ & $\mathbf{3 0}$ \\
\hline 2 & 2.0 & 2.3 & 2.5 \\
10 & 1.8 & 2.1 & 2.2 \\
\hline
\end{tabular}




\section{RÍO LOCO BASIN}

\section{Quebrada Grande upstream from Lago Loco, Puerto Rico}

LOCATION.--Lat 1803'45", long 66 53'10", Hydrologic Unit 21010004, at barrio Almácigo Alto, $800 \mathrm{ft}$ (244 m) upstream from confluence with Río Loco,

$1.2(1.9 \mathrm{~km})$ north from spillway, and $3.0 \mathrm{mi}(4.8 \mathrm{~km})$ northwest from Plaza de Yauco.

DRAINAGE AREA.--2.7 $\mathrm{mi}^{2}\left(7.0 \mathrm{~km}^{2}\right)$.

LOW-FLOW ESTIMATES.--Based on correlation of 8 base-flow measurements with concurrent base flows at gaging station 50124200 .

MAGNITUDE AND FREQUENCY OF ANNUAL LOW FLOWS

\begin{tabular}{cccc}
\hline $\begin{array}{c}\text { Recurrence } \\
\text { interval, } \\
\text { in years }\end{array}$ & \multicolumn{2}{c}{$\begin{array}{c}\text { Lowest average flow, in cubic feet per second, } \\
\text { for indicated number of consecutive days }\end{array}$} \\
\cline { 2 - 4 } 2 & $\mathbf{7}$ & $\mathbf{1 4}$ & $\mathbf{3 0}$ \\
\hline 10 & .4 & .4 & .5 \\
\hline
\end{tabular}

50128500 Río Loco upstream from Lago Loco, Puerto Rico

LOCATION.--Lat $18^{\circ} 03^{\prime 2} 22^{\prime \prime}$, long 66 53'08", Hydrologic Unit 21010004, at barrio Susúa Alta, $0.2 \mathrm{mi}(0.3 \mathrm{~km})$ upstream from Lago Loco, $1.9 \mathrm{mi}(3.0 \mathrm{~km})$ northeast from Cerro La Torre, and $5.2 \mathrm{mi}(8.4 \mathrm{~km})$ southeast from Plaza de Sabana Grande.

DRAINAGE AREA.-- $7.7 \mathrm{mi}^{2}\left(20 \mathrm{~km}^{2}\right)$.

LOW-FLOW ESTIMATES.--Based on correlation of 8 base-flow measurements with concurrent base flows at gaging station 50124200.

MAGNITUDE AND FREQUENCY OF ANNUAL LOW FLOWS

\begin{tabular}{cccc}
\hline $\begin{array}{c}\text { Recurrence } \\
\text { interval, }\end{array}$ & \multicolumn{2}{c}{$\begin{array}{c}\text { Lowest average flow, in cubic feet per second, } \\
\text { for indicated number of consecutive days }\end{array}$} \\
\cline { 2 - 4 } in years & $\mathbf{7}$ & $\mathbf{1 4}$ & $\mathbf{3 0}$ \\
\hline 2 & .9 & 1.1 & 1.3 \\
10 & .7 & .9 & 1.0 \\
\hline
\end{tabular}

50129200 Quebrada Susúa at Palomas, Puerto Rico

LOCATION.--Lat $18^{\circ} 01^{\prime} 19^{\prime \prime}$, long 66 52'28", Hydrologic Unit 21010004, on bridge at Highway 2, $0.5 \mathrm{mi}(0.8 \mathrm{~km})$ north of Palomas, and $1.9 \mathrm{mi}(3.1 \mathrm{~km})$ southwest of Yauco.

DRAINAGE AREA.-- $3.2 \mathrm{mi}^{2}\left(8.4 \mathrm{~km}^{2}\right)$.

LOW-FLOW ESTIMATES.--Based on correlation of 8 base-flow measurements with concurrent base flows at gaging station 50124200 .

MAGNITUDE AND FREQUENCY OF ANNUAL LOW FLOWS

\begin{tabular}{cccc}
\hline $\begin{array}{c}\text { Recurrence } \\
\text { interval, } \\
\text { in years }\end{array}$ & \multicolumn{3}{c}{$\begin{array}{c}\text { Lowest average flow, in cubic feet per second, } \\
\text { for indicated number of consecutive days }\end{array}$} \\
\cline { 2 - 4 } 2 & $\mathbf{7}$ & $\mathbf{1 4}$ & $\mathbf{3 0}$ \\
\hline 10 & .3 & .4 & .4 \\
& .2 & .3 & .3 \\
\hline
\end{tabular}




\section{RÍO GUANAJIBO BASIN}

\section{Río Grande near Sabana Grande, Puerto Rico}

LOCATION.--Lat $18^{\circ} 05^{\prime} 53^{\prime \prime}$, long 66 56' 18", Hydrologic Unit 21010003, at barrio Rín on Highway 364, 0.5 mi (0.8 km) northeast from Capilla del Pozo de La Virgen, and $1.8 \mathrm{mi}(2.9 \mathrm{~km})$ northeast from Plaza de Sabana Grande.

DRAINAGE AREA.-- $6.4 \mathrm{mi}^{2}\left(17 \mathrm{~km}^{2}\right)$.

LOW-FLOW ESTIMATES.--Based on correlation of 8 base-flow measurements with concurrent base flows at gaging station 50138000 .

MAGNITUDE AND FREQUENCY OF ANNUAL LOW FLOWS

\begin{tabular}{cccc}
\hline $\begin{array}{c}\text { Recurrence } \\
\text { interval, }\end{array}$ & \multicolumn{2}{c}{$\begin{array}{c}\text { Lowest average flow, in cubic feet per second, } \\
\text { for indicated number of consecutive days }\end{array}$} \\
\cline { 2 - 4 } in years & $\mathbf{7}$ & $\mathbf{1 4}$ & $\mathbf{3 0}$ \\
\hline 2 & .4 & .4 & .7 \\
10 & .2 & .2 & .3 \\
\hline
\end{tabular}

50130500 Río Guanajibo at La Pica, Puerto Rico

LOCATION.--Lat $18^{\circ} 04^{\prime} 11^{\prime \prime}$, long 6657'29", Hydrologic Unit 21010003, at barrio Rayo on Highway 2, 1.0 mi (1.6 km) north from Cerro de Los Bonelli, and $0.8 \mathrm{mi}(1.3 \mathrm{~km})$ southeast from Plaza de Sabana Grande.

DRAINAGE AREA.-- $15 \mathrm{mi}^{2}\left(38 \mathrm{~km}^{2}\right)$.

LOW-FLOW ESTIMATES.--Based on correlation of 8 base-flow measurements with concurrent base flows at gaging station 50138000 .

MAGNITUDE AND FREQUENCY OF ANNUAL LOW FLOWS

\begin{tabular}{cccc}
\hline $\begin{array}{c}\text { Recurrence } \\
\text { interval, }\end{array}$ & \multicolumn{2}{c}{$\begin{array}{c}\text { Lowest average flow, in cubic feet per second, } \\
\text { for indicated number of consecutive days }\end{array}$} \\
\cline { 2 - 4 } in years & $\mathbf{7}$ & $\mathbf{1 4}$ & $\mathbf{3 0}$ \\
\hline 2 & 1.2 & 1.4 & 1.8 \\
10 & .6 & .7 & 1.0 \\
\hline
\end{tabular}

50130800 Río Flores near Sabana Grande, Puerto Rico

LOCATION.--Lat $18^{\circ} 04^{\prime} 02^{\prime \prime}$, long 66 $58^{\prime} 25^{\prime \prime}$, Hydrologic Unit 21010003, at barrio Santana on Highway 2, $0.2 \mathrm{mi}(0.3 \mathrm{~km})$ east from intersection of Highways 2 and 363, and $0.9 \mathrm{mi}(1.4 \mathrm{~km})$ west from Plaza de Sabana Grande.

DRAINAGE AREA.--2.0 $\mathrm{mi}^{2}\left(5.1 \mathrm{~km}^{2}\right)$.

LOW-FLOW ESTIMATES.--Based on correlation of 8 base-flow measurements with concurrent base flows at gaging station 50138000 .

MAGNITUDE AND FREQUENCY OF ANNUAL LOW FLOWS

\begin{tabular}{cccc}
\hline $\begin{array}{c}\text { Recurrence } \\
\text { interval, }\end{array}$ & \multicolumn{2}{c}{$\begin{array}{l}\text { Lowest average flow, in cubic feet per second, } \\
\text { for indicated number of consecutive days }\end{array}$} \\
\cline { 2 - 4 } in years & $\mathbf{7}$ & $\mathbf{1 4}$ & $\mathbf{3 0}$ \\
\hline 2 & $<.1$ & $<.1$ & $<.1$ \\
10 & $<.1$ & $<.1$ & $<.1$ \\
\hline
\end{tabular}

50131010 Río Cruses near Sabana Grande, Puerto Rico

LOCATION.--Lat $18^{\circ} 04^{\prime} 54^{\prime \prime}$, long 66 58'37", Hydrologic Unit 21010003, at barrio Santana on Highway 2, $400 \mathrm{ft}$ (122 m) west from intersection of Highways 2 and 363, and $1.1 \mathrm{mi}(1.8 \mathrm{~km})$ west from Plaza de Sabana Grande.

DRAINAGE AREA.--4.7 $\mathrm{mi}^{2}\left(12 \mathrm{~km}^{2}\right)$.

LOW-FLOW ESTIMATES.--Based on correlation of 8 base-flow measurements with concurrent base flows at gaging station 50138000 .

REMARKS.--Minor diversions are made above the station for public-water supply.

MAGNITUDE AND FREQUENCY OF ANNUAL LOW FLOWS

\begin{tabular}{cccc}
\hline $\begin{array}{c}\text { Recurrence } \\
\text { interval, }\end{array}$ & \multicolumn{2}{c}{$\begin{array}{c}\text { Lowest average flow, in cubic feet per second, } \\
\text { for indicated number of consecutive days }\end{array}$} \\
\cline { 2 - 4 } in years & $\mathbf{7}$ & $\mathbf{1 4}$ & $\mathbf{3 0}$ \\
\hline 2 & .9 & 1.0 & 1.1 \\
10 & .5 & .6 & .8 \\
\hline
\end{tabular}




\section{RÍO GUANAJIBO BASIN-Continued}

\section{Río Cupeyes near San Germán, Puerto Rico}

LOCATION.--Lat $18^{\circ} 04^{\prime} 48^{\prime \prime}$, long $67^{\circ} 00^{\prime} 24^{\prime \prime}$, Hydrologic Unit 21010003, at barrio Guamá, $0.2 \mathrm{mi}(0.3 \mathrm{~km})$ downstream from Highway 2 , and $2.5 \mathrm{mi}$ (4.0 $\mathrm{km}$ ) east from Plaza de San Germán.

DRAINAGE AREA.-- $4.2 \mathrm{mi}^{2}\left(11 \mathrm{~km}^{2}\right)$.

LOW-FLOW ESTIMATES.--Based on correlation of 8 base-flow measurements with concurrent base flows at gaging station 50138000 .

MAGNITUDE AND FREQUENCY OF ANNUAL LOW FLOWS

\begin{tabular}{cccc}
\hline $\begin{array}{c}\text { Recurrence } \\
\text { interval, } \\
\text { in years }\end{array}$ & \multicolumn{2}{c}{$\begin{array}{c}\text { Lowest average flow, in cubic feet per second, } \\
\text { for indicated number of consecutive days }\end{array}$} \\
\cline { 2 - 4 } 2 & $\mathbf{7}$ & $\mathbf{1 4}$ & $\mathbf{3 0}$ \\
\hline 10 & .6 & .7 & .8 \\
\hline
\end{tabular}

50132010 Río Guanajibo below San Germán, Puerto Rico

LOCATION.--Lat $18^{\circ} 05^{\prime 2} 28^{\prime \prime}$, long $67^{\circ} 02^{\prime} 38^{\prime \prime}$, Hydrologic Unit $21010003,1,500 \mathrm{ft}(457 \mathrm{~m})$ downstream from bridge on Highway $360,0.5 \mathrm{mi}(0.8 \mathrm{~km})$ south from Plaza de San Germán.

DRAINAGE AREA.--36 $\mathrm{mi}^{2}\left(94 \mathrm{~km}^{2}\right)$.

LOW-FLOW ESTIMATES.--Based on correlation of 8 base-flow measurements with concurrent base flows at gaging station 50138000.

REMARKS.--Minor diversions are made above the station for public-water supply.

MAGNITUDE AND FREQUENCY OF ANNUAL LOW FLOWS

\begin{tabular}{cccc}
\hline $\begin{array}{c}\text { Recurrence } \\
\text { interval, }\end{array}$ & \multicolumn{2}{c}{$\begin{array}{c}\text { Lowest average flow, in cubic feet per second, } \\
\text { for indicated number of consecutive days }\end{array}$} \\
\cline { 2 - 4 } in years & $\mathbf{7}$ & $\mathbf{1 4}$ & $\mathbf{3 0}$ \\
\hline 2 & 4.4 & 5.3 & 6.3 \\
10 & 2.3 & 2.9 & 3.9 \\
\hline
\end{tabular}

50133000 Río Caín near San Germán, Puerto Rico

LOCATION.--Lat 1806'06", long 6702'26", Hydrologic Unit 21010003, at Barrio Caín on Highway 361, $600 \mathrm{ft}$ (183 m) upstream from Highway 2, and $1.3 \mathrm{mi}(2.1 \mathrm{~km})$ north from Plaza de San Germán.

DRAINAGE AREA.--6.3 $\mathrm{mi}^{2}\left(16 \mathrm{~km}^{2}\right)$.

LOW-FLOW ESTIMATES.--Based on correlation of 8 base-flow measurements with concurrent base flows at gaging station 50138000.

MAGNITUDE AND FREQUENCY OF ANNUAL LOW FLOWS

\begin{tabular}{cccc}
\hline $\begin{array}{c}\text { Recurrence } \\
\text { interval, } \\
\text { in years }\end{array}$ & \multicolumn{2}{c}{$\begin{array}{c}\text { Lowest average flow, in cubic feet per second, } \\
\text { for indicated number of consecutive days }\end{array}$} \\
\cline { 2 - 4 } 2 & $\mathbf{7}$ & $\mathbf{1 4}$ & $\mathbf{3 0}$ \\
\hline 10 & .2 & .3 & .4 \\
\hline
\end{tabular}




\section{RÍO GUANAJIBO BASIN-Continued}

\section{Río Duey near Rosario, Puerto Rico}

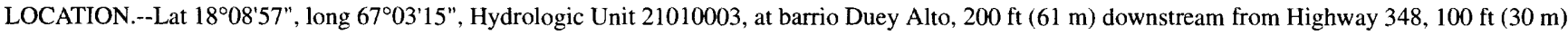
downstream from confluence with Río Nueve Pasos, and $2.0 \mathrm{mi}(3.2 \mathrm{~km})$ southeast from Plaza de Rosario.

DRAINAGE AREA.--4.2 $\mathrm{mi}^{2}\left(11 \mathrm{~km}^{2}\right)$.

LOW-FLOW ESTIMATES.--Based on correlation of 8 base-flow measurements with concurrent base flows at gaging station 50138000 .

REMARKS.--Minor diversions are made above the station for public-water supply.

MAGNITUDE AND FREQUENCY OF ANNUAL LOW FLOWS

\begin{tabular}{cccc}
\hline $\begin{array}{c}\text { Recurrence } \\
\text { interval, }\end{array}$ & \multicolumn{2}{c}{$\begin{array}{c}\text { Lowest average flow, in cubic feet per second, } \\
\text { for indicated number of consecutive days }\end{array}$} \\
\cline { 2 - 4 } in years & $\mathbf{7}$ & $\mathbf{1 4}$ & $\mathbf{3 0}$ \\
\hline 2 & .9 & 1.1 & 1.2 \\
10 & .6 & .7 & .9 \\
\hline
\end{tabular}

50134600 Río Hoconuco near San Germán, Puerto Rico

LOCATION.--Lat $18^{\circ} 07^{\prime} 08^{\prime \prime}$, long $67^{\circ} 04^{\prime} 27^{\prime \prime}$, Hydrologic Unit 21010003 , at barrio Hoconuco Bajo, $0.2 \mathrm{mi}(0.3 \mathrm{~km})$ downstream from Highway $358,200 \mathrm{ft}$ upstream from confluence with Río Duey, and $3.2 \mathrm{mi}(5.1 \mathrm{~km})$ northeast from Plaza de San Germán.

DRAINAGE AREA.--5.2 $\mathrm{mi}^{2}\left(13 \mathrm{~km}^{2}\right)$.

LOW-FLOW ESTIMATES.--Based on correlation of 8 base-flow measurements with concurrent base flows at gaging station 50138000 .

MAGNITUDE AND FREQUENCY OF ANNUAL LOW FLOWS

\begin{tabular}{cccc}
\hline $\begin{array}{c}\text { Recurrence } \\
\text { interval, } \\
\text { in years }\end{array}$ & \multicolumn{3}{c}{$\begin{array}{c}\text { Lowest average flow, in cubic feet per second, } \\
\text { for indicated number of consecutive days }\end{array}$} \\
\cline { 2 - 4 } & \multicolumn{7}{|c}{} & $\mathbf{1 4}$ & $\mathbf{3 0}$ \\
\hline 2 & $<.1$ & .1 & .2 \\
10 & $<.1$ & $<.1$ & $<.1$ \\
\hline
\end{tabular}

50135000 Río Hoconuco (Duey) near San Germán, Puerto Rico

LOCATION.--Lat $18^{\circ} 07^{\prime} 10^{\prime \prime}$, long 67 $04^{\prime} 48^{\prime \prime}$, Hydrologic Unit 21010003, at barrio Duey Bajo, $200 \mathrm{ft}(61 \mathrm{~m})$ downstream from Highway 2 , and $3.4 \mathrm{mi}(5.5$ $\mathrm{km})$ northwest from Plaza de San Germán.

DRAINAGE AREA.--13 $\mathrm{mi}^{2}\left(34 \mathrm{~km}^{2}\right)$.

LOW-FLOW ESTIMATES.--Based on correlation of 8 base-flow measurements with concurrent base flows at gaging station 50138000 .

MAGNITUDE AND FREQUENCY OF ANNUAL LOW FLOWS

\begin{tabular}{cccc}
\hline $\begin{array}{c}\text { Recurrence } \\
\text { interval, } \\
\text { in years }\end{array}$ & $\begin{array}{c}\text { Lowest average flow, in cubic feet per second, } \\
\text { for indicated number of consecutive days }\end{array}$ \\
\cline { 2 - 3 } 2 & $\mathbf{7}$ & $\mathbf{1 4}$ & $\mathbf{3 0}$ \\
\hline 10 & .8 & 1.0 & 1.2 \\
.4 & .5 & .7 \\
\hline
\end{tabular}




\section{RÍO GUANAJIBO BASIN-Continued \\ 50135700 Río Maricao at Maricao, Puerto Rico}

LOCATION.--Lat $18^{\circ} 11^{\prime 2} 2^{\prime \prime}$, long 6659'37", Hydrologic Unit 21010003, at barrio Maricao Afuera on Highway $357,0.4 \mathrm{mi}(0.6 \mathrm{~km})$ east from Hacienda San Antonio, and $1.0 \mathrm{mi}(1.6 \mathrm{~km})$ northwest from Plaza de Maricao.

DRAINAGE AREA.--3.8 $\mathrm{mi}^{2}\left(9.8 \mathrm{~km}^{2}\right)$.

LOW-FLOW ESTIMATES.--Based on correlation of 8 base-flow measurements with concurrent base flows at gaging station 50136000 .

REMARKS.--Minor diversions are made above the station for public-water supply.

$$
\text { MAGNITUDE AND FREQUENCY OF ANNUAL LOW FLOWS }
$$

\begin{tabular}{cccc}
\hline $\begin{array}{c}\text { Recurrence } \\
\text { interval, }\end{array}$ & \multicolumn{2}{c}{$\begin{array}{c}\text { Lowest average flow, in cubic feet per second, } \\
\text { for indicated number of consecutive days }\end{array}$} \\
\cline { 2 - 4 } in years & $\mathbf{7}$ & $\mathbf{1 4}$ & $\mathbf{3 0}$ \\
\hline 2 & 2.8 & 3.0 & 3.2 \\
10 & 1.6 & 1.8 & 2.0 \\
\hline
\end{tabular}

50135800 Río Rosario at Las Vegas, Puerto Rico

LOCATION.--Lat $18^{\circ} 11^{\prime} 13^{\prime \prime}$, long $67^{\circ} 01^{\prime} 52^{\prime \prime}$, Hydrologic Unit 21010003 , at barrio Montoso on Highway $119,0.1 \mathrm{mi}(0.2 \mathrm{~km})$ southeast from intersection of Highways 119 and 105, and $3.6 \mathrm{mi}(5.8 \mathrm{~km})$ northeast from Plaza de Rosario.

DRAINAGE AREA.--8.3 $\mathrm{mi}^{2}\left(22 \mathrm{~km}^{2}\right)$.

LOW-FLOW ESTIMATES.--Based on correlation of 8 base-flow measurements with concurrent base flows at gaging station 50136000 .

REMARKS.--Minor diversions are made above the station for public-water supply.

MAGNITUDE AND FREQUENCY OF ANNUAL LOW FLOWS

\begin{tabular}{cccc}
\hline $\begin{array}{c}\text { Recurrence } \\
\text { interval, }\end{array}$ & \multicolumn{2}{c}{$\begin{array}{c}\text { Lowest average flow, in cubic feet per second, } \\
\text { for indicated number of consecutive days }\end{array}$} \\
\cline { 2 - 4 } in years & $\mathbf{7}$ & $\mathbf{1 4}$ & $\mathbf{3 0}$ \\
\hline 2 & 6.0 & 6.1 & 6.4 \\
10 & 3.2 & 3.5 & 4.0 \\
\hline
\end{tabular}

50136400 Río Rosario near Hormigueros, Puerto Rico

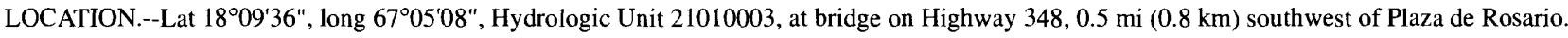
DRAINAGE AREA.-- $18 \mathrm{mi}^{2}\left(47 \mathrm{~km}^{2}\right)$.

LOW-FLOW ESTIMATES.--Based on correlation of 8 base-flow measurements with concurrent base flows at gaging station 50136000.

REMARKS.--Minor diversions are made above the station for public-water supply.

MAGNITUDE AND FREQUENCY OF ANNUAL LOW FLOWS

\begin{tabular}{cccc}
\hline $\begin{array}{c}\text { Recurrence } \\
\text { interval, }\end{array}$ & \multicolumn{2}{c}{$\begin{array}{c}\text { Lowest average flow, in cubic feet per second, } \\
\text { for indicated }\end{array}$} \\
\cline { 2 - 4 } in years & $\mathbf{7}$ & $\mathbf{1 4}$ & $\mathbf{3 0}$ \\
\hline 2 & 9.8 & 11 & 11 \\
10 & 5.0 & 5.4 & 6.3 \\
\hline
\end{tabular}




\section{RÍO GUANAJIBO BASIN-Continued \\ 50136500 Río Rosario near Hormigueros, Puerto Rico}

LOCATION.--Lat $18^{\circ} 07^{\prime} 35^{\prime \prime}$, long 67 $05^{\prime} 39^{\prime \prime}$, Hydrologic Unit 21010003, at barrio Benavente on Highway $2,2.7 \mathrm{mi}(4.3 \mathrm{~km})$ southwest from Rosario, and $2.5 \mathrm{mi}(4.0 \mathrm{~km})$ southwest from Plaza de Hormigueros.

DRAINAGE AREA.--23 $\mathrm{mi}^{2}\left(59 \mathrm{~km}^{2}\right)$.

LOW-FLOW ESTIMATES.--Based on correlation of 8 base-flow measurements with concurrent base flows at gaging station 50136000 .

REMARKS.--Minor diversions are made above the station for public-water supply.

MAGNITUDE AND FREQUENCY OF ANNUAL LOW FLOWS

\begin{tabular}{cccc}
\hline $\begin{array}{c}\text { Recurrence } \\
\text { interval, }\end{array}$ & \multicolumn{2}{c}{$\begin{array}{c}\text { Lowest average flow, in cubic feet per second, } \\
\text { for indicated number of consecutive days }\end{array}$} \\
\cline { 2 - 4 } in years & $\mathbf{7}$ & $\mathbf{1 4}$ & $\mathbf{3 0}$ \\
\hline 2 & 9.0 & 9.8 & 10 \\
10 & 4.4 & 4.6 & 5.6 \\
\hline
\end{tabular}

50137800 Río Viejo near Cabo Rojo, Puerto Rico

LOCATION.--Lat $18^{\circ} 06^{\prime} 04^{\prime \prime}$, long $67^{\circ} 07^{\prime} 48^{\prime \prime}$, Hydrologic Unit 21010003 , at barrio Bajura on Highway $103,1.0 \mathrm{mi}(1.6 \mathrm{~km})$ northeast from intersection with Highway 102, and $1.4 \mathrm{mi}(2.2 \mathrm{~km})$ from Plaza de Cabo Rojo.

DRAINAGE AREA.-- $12 \mathrm{mi}^{2}\left(32 \mathrm{~km}^{2}\right)$.

LOW-FLOW ESTIMATES.--Based on correlation of 8 base-flow measurements with concurrent base flows at gaging station 50138000 .

MAGNITUDE AND FREQUENCY OF ANNUAL LOW FLOWS

\begin{tabular}{cccc}
\hline $\begin{array}{c}\text { Recurrence } \\
\text { interval, } \\
\text { in years }\end{array}$ & \multicolumn{2}{c}{$\begin{array}{c}\text { Lowest average flow, in cubic feet per second, } \\
\text { for indicated number of consecutive days }\end{array}$} \\
\cline { 2 - 4 } 2 & $\mathbf{7}$ & $\mathbf{1 4}$ & $\mathbf{3 0}$ \\
\hline 10 & 1.2 & 1.5 & 1.8 \\
& .6 & .8 & 1.1 \\
\hline
\end{tabular}

QUEBRADA MAGA BASIN

50138100 Quebrada Maga near Guanajibo, Puerto Rico

LOCATION.--Lat $18^{\circ} 09^{\prime} 18^{\prime \prime}$, long 67 $08^{\prime} 07^{\prime \prime}$, Hydrologic Unit 21010003, at barrio Guanajibo, $0.3 \mathrm{mi}(0.5 \mathrm{~km})$ southeast from Mayagüez Mall, and $1.2 \mathrm{mi}$ $(1.9 \mathrm{~km})$ northwest from Plaza de Hormigueros.

DRAINAGE AREA.--0.8 $\mathrm{mi}^{2}\left(2.0 \mathrm{~km}^{2}\right)$.

REMARKS.--Estimated minimum average 7-, 14-, and 30-day flow with 2- and 10-year recurrence interval is 0.0 on the basis of 8 observation of no flow under base-flow conditions. 


\section{RIOO HONDO BASIN}

\section{Río Hondo near Guanajibo, Puerto Rico}

LOCATION.--Lat $18^{\circ} 09^{\prime} 45^{\prime \prime}$, long 67\%09'00", Hydrologic Unit 21010003 , at barrio Guanajibo on Highway 114 , and $1.8 \mathrm{mi}(2.9 \mathrm{~km})$ east from Cerro Cornelia, and $2.0(3.2 \mathrm{~km})$ northwest from Plaza de Hormigueros.

DRAINAGE AREA.-- $3.2 \mathrm{mi}^{2}\left(8.2 \mathrm{~km}^{2}\right)$.

LOW-FLOW ESTIMATES.--Based on correlation of 8 base-flow measurements with concurrent base flows at gaging station 50138000 .

MAGNITUDE AND FREQUENCY OF ANNUAL LOW FLOWS

\begin{tabular}{cccc}
\hline $\begin{array}{c}\text { Recurrence } \\
\text { interval, } \\
\text { in years }\end{array}$ & \multicolumn{2}{c}{$\begin{array}{c}\text { Lowest average flow, in cubic feet per second, } \\
\text { for indicated number of consecutive days }\end{array}$} \\
\cline { 2 - 4 } 2 & $\mathbf{7}$ & $\mathbf{1 4}$ & $\mathbf{3 0}$ \\
\hline 10 & .2 & .3 & .4 \\
& .1 & .1 & .2 \\
\hline \multirow{4}{*}{ QUEBRADA SÁBALOS BASIN } \\
$\mathbf{5 0 1 3 8 3 0 0}$ Quebrada Sábalos near Mayagüez, Puerto Rico
\end{tabular}

LOCATION.--Lat $18^{\circ} 10^{\prime} 47^{\prime \prime}$, long 6708'58", Hydrologic Unit 21010003, at barrio Sábalos on Highway 2, 2.9 mi (4.7 km) northwest from Hormigueros, and $1.7 \mathrm{mi}(2.7 \mathrm{~km})$ southwest from Plaza de Mayagüez.

DRAINAGE AREA.-- $2.5 \mathrm{mi}^{2}\left(6.4 \mathrm{~km}^{2}\right)$.

LOW-FLOW ESTIMATES.--Based on correlation of 8 base-flow measurements with concurrent base flows at gaging station 50138000 .

MAGNITUDE AND FREQUENCY OF ANNUAL LOW FLOWS

\begin{tabular}{cccc}
\hline $\begin{array}{c}\text { Recurrence } \\
\text { interval, }\end{array}$ & \multicolumn{2}{c}{$\begin{array}{c}\text { Lowest average flow, in cubic feet per second, } \\
\text { for indicated number of consecutive days }\end{array}$} \\
\cline { 2 - 4 } in years & $\mathbf{7}$ & $\mathbf{1 4}$ & $\mathbf{3 0}$ \\
\hline 2 & .3 & .4 & .5 \\
10 & .2 & .2 & .3 \\
\hline & RiO YAGÜEZ BASIN
\end{tabular}

\section{Río Yagüez at Balboa, Puerto Rico}

LOCATION.--Lat $18^{\circ} 12^{\prime} 13^{\prime \prime}$, long 6707'55", Hydrologic Unit 21010003, about 1,200 ft (366 m) upstream from bridge on Balboa St., and 1.6 (2.6 km) upstream from mouth.

DRAINAGE AREA.-- $12 \mathrm{mi}^{2}\left(32 \mathrm{~km}^{2}\right)$.

LOW-FLOW ESTIMATES.--Based on correlation of 8 base-flow measurements with concurrent base-flows at gaging station 50136000 .

REMARKS.--Minor diversions are made above station to filter plant for public-water supply.

MAGNITUDE AND FREQUENCY OF ANNUAL LOW FLOWS

\begin{tabular}{cccc}
\hline $\begin{array}{c}\text { Recurrence } \\
\text { interval, }\end{array}$ & \multicolumn{2}{c}{$\begin{array}{c}\text { Lowest average flow, in cubic feet per second, } \\
\text { for indicated number of consecutive days }\end{array}$} \\
\cline { 2 - 4 } in years & $\mathbf{7}$ & $\mathbf{1 4}$ & $\mathbf{3 0}$ \\
\hline 2 & 3.6 & 4.2 & 4.4 \\
10 & $\mathbf{1 . 6}$ & 1.8 & 2.2 \\
\hline
\end{tabular}




\section{RÍO GRANDE DE AÑASCO BASIN}

\section{Río Guilarte near Adjuntas, Puerto Rico}

LOCATION.--Lat $18^{\circ} 10^{\prime} 58^{\prime \prime}$, long $66^{\circ} 46^{\prime} 09^{\prime \prime}$, Hydrologic Unit 21010003 , at barrio Guilarte on Highway $131,0.4$ mi $(0.6 \mathrm{~km})$ southwest from intersection of Highways 130 and 131 , and $4.3 \mathrm{mi}(6.9 \mathrm{~km})$ east from Castañer.

DRAINAGE AREA.--2.6 $\mathrm{mi}\left(6.8 \mathrm{~km}^{2}\right)$.

LOW-FLOW ESTIMATES.--Based on correlation of 8 base-flow measurements with concurrent base flows at gaging station 50141000 .

\section{MAGNITUDE AND FREQUENCY OF ANNUAL LOW FLOWS}

\begin{tabular}{cccc}
\hline $\begin{array}{c}\text { Recurrence } \\
\text { interval, }\end{array}$ & \multicolumn{2}{c}{$\begin{array}{c}\text { Lowest average flow, in cubic feet per second, } \\
\text { for indicated number of consecutive days }\end{array}$} \\
\cline { 2 - 4 } in years & $\mathbf{7}$ & $\mathbf{1 4}$ & $\mathbf{3 0}$ \\
\hline 2 & 1.7 & 1.8 & 2.0 \\
10 & 1.3 & 1.4 & 1.5 \\
\hline
\end{tabular}

50140800 Río Limani near Adjuntas, Puerto Rico

LOCATION.--Lat $18^{\circ} 12^{\prime} 01^{\prime \prime}$, long 66 $47^{\prime} 50^{\prime \prime}$, Hydrologic Unit 21010003, at barrio Yahuecas, $200 \mathrm{ft}$ (61 m) upstream from confluence with Río Guilarte, and $500 \mathrm{ft}(152 \mathrm{~m})$ southwest from intersection of Highways 129 and 135.

DRAINAGE AREA.-- $7.4 \mathrm{mi}^{2}\left(19 \mathrm{~km}^{2}\right)$.

LOW-FLOW ESTIMATES.--Based on correlation of 8 base-flow measurements with concurrent base flows at gaging station 50141000 .

MAGNITUDE AND FREQUENCY OF ANNUAL LOW FLOWS

\begin{tabular}{cccc}
\hline $\begin{array}{c}\text { Recurrence } \\
\text { interval, }\end{array}$ & \multicolumn{2}{c}{$\begin{array}{c}\text { Lowest average flow, in cubic feet per second, } \\
\text { for indicated number of consecutive days }\end{array}$} \\
\cline { 2 - 4 } in years & $\mathbf{7}$ & $\mathbf{1 4}$ & $\mathbf{3 0}$ \\
\hline 2 & 3.5 & 3.7 & 4.2 \\
10 & 1.5 & 1.7 & 3.0 \\
\hline
\end{tabular}

50141400 Río Guayo at Guayo, Puerto Rico

LOCATION.--Lat $18^{\circ} 10^{\prime} 49^{\prime \prime}$, long $66^{\circ} 49^{\prime} 40^{\prime \prime}$, Hydrologic Unit 21010003, Barrio Guayo on Highway $131,1.0 \mathrm{mi}(1.6 \mathrm{~km})$ upstream from Lago Guayo, 0.4 $\mathrm{mi}(0.6 \mathrm{~km})$ southeast from Castañer.

DRAINAGE AREA.-- $4.2 \mathrm{mi}^{2}\left(11 \mathrm{~km}^{2}\right)$.

LOW-FLOW ESTIMATES.--Based on correlation of 8 base-flow measurements with concurrent base flows at gaging station 50141000 .

MAGNITUDE AND FREQUENCY OF ANNUAL LOW FLOWS

\begin{tabular}{cccc}
\hline $\begin{array}{c}\text { Recurrence } \\
\text { interval, } \\
\text { in years }\end{array}$ & \multicolumn{2}{c}{$\begin{array}{c}\text { Lowest average flow, in cubic feet per second, } \\
\text { for indicated number of consecutive days }\end{array}$} \\
\cline { 2 - 4 } 2 & $\mathbf{7}$ & $\mathbf{1 4}$ & $\mathbf{3 0}$ \\
\hline 10 & 2.0 & 2.1 & 2.4 \\
& 1.5 & 1.6 & 1.8 \\
\hline
\end{tabular}




\section{RÍO GRANDE DE AÑASCO BASIN-Continued \\ 50142000 Río Blanco at La Torre, Puerto Rico}

LOCATION.--Lat 18 $18^{\prime} 34^{\prime \prime}$, long 66 $51^{\prime} 49^{\prime \prime}$, Hydrologic Unit 21010003, at barrio La Torre on Highway 128, 2.7 mi (4.3 km) northwest from Lago Guayo, and $4.5 \mathrm{mi}(7.2 \mathrm{~km})$ northwest from Castañer.

DRAINAGE AREA. $-6.2 \mathrm{mi}^{2}\left(16 \mathrm{~km}^{2}\right)$. Does not include $27 \mathrm{mi}^{2}\left(69.9 \mathrm{~km}^{2}\right)$ in which base flow is diverted to Lago Lucchetti Dam.

LOW-FLOW ESTIMATES.--Based on correlation of 8 base-flow measurements with concurrent base flows at gaging station 50144000 .

REMARKS.--Flow regulated by Lago Yahuecas and Lago Guayo Dams.

MAGNITUDE AND FREQUENCY OF ANNUAL LOW FLOWS

\begin{tabular}{cccc}
\hline $\begin{array}{c}\text { Recurrence } \\
\text { interval, }\end{array}$ & \multicolumn{2}{c}{$\begin{array}{c}\text { Lowest average flow, in cubic feet per second, } \\
\text { for indicated number of consecutive days }\end{array}$} \\
\cline { 2 - 4 } in years & $\mathbf{7}$ & $\mathbf{1 4}$ & $\mathbf{3 0}$ \\
\hline 2 & 2.4 & 2.7 & 3.0 \\
10 & 1.6 & 1.8 & 2.2 \\
\hline
\end{tabular}

50142100 Quebrada de Los Plátanos at Marisol, Puerto Rico

LOCATION.--Lat $18^{\circ} 15^{\prime} 41^{\prime \prime}$, long 66 51'22", Hydrologic Unit 21010003, at Barrio Marisol on Highway 128, $0.3 \mathrm{mi}(0.5 \mathrm{~km})$ south from intersection of Highways 128 and 129.

DRAINAGE AREA.--0.6 $\mathrm{mi}^{2}\left(1.5 \mathrm{~km}^{2}\right)$.

LOW-FLOW ESTIMATES.--Based on correlation of 8 base-flow measurements with concurrent base flows at gaging station 50144000 .

MAGNITUDE AND FREQUENCY OF ANNUAL LOW FLOWS

\begin{tabular}{cccc}
\hline $\begin{array}{c}\text { Recurrence } \\
\text { interval, } \\
\text { in years }\end{array}$ & \multicolumn{2}{c}{$\begin{array}{c}\text { Lowest average flow, in cubic feet per second, } \\
\text { for indicated number of consecutive days }\end{array}$} \\
\cline { 2 - 4 } & $\mathbf{7}$ & $\mathbf{1 4}$ & $\mathbf{3 0}$ \\
\hline 2 & .2 & .2 & .2 \\
10 & .1 & .2 & .2 \\
\hline
\end{tabular}

50142300 Río Prieto at Indiera Alta, Puerto Rico

LOCATION.--Lat $18^{\circ} 10^{\prime} 07^{\prime \prime}$, long 66 51'49", Hydrologic Unit 21010003, at barrio Indiera Alta on Highway 128, 2.3 mi (3.7 km) southwest from Lago Guayo, and $2.2 \mathrm{mi}(3.5 \mathrm{~km})$ southwest from Castañer.

DRAINAGE AREA.--7.5 $\mathrm{mi}^{2}\left(19 \mathrm{~km}^{2}\right)$.

LOW-FLOW ESTIMATES.--Based on correlation of 8 base-flow measurements with concurrent base flows at gaging station 50141000 .

MAGNITUDE AND FREQUENCY OF ANNUAL LOW FLOWS

\begin{tabular}{cccc}
\hline $\begin{array}{c}\text { Recurrence } \\
\text { interval, }\end{array}$ & \multicolumn{2}{c}{$\begin{array}{c}\text { Lowest average flow, in cubic feet per second, } \\
\text { for indicated number of consecutive days }\end{array}$} \\
\cline { 2 - 4 } in years & $\mathbf{7}$ & $\mathbf{1 4}$ & $\mathbf{3 0}$ \\
\hline 2 & 4.1 & 4.4 & 4.8 \\
10 & 3.0 & 3.3 & 3.6 \\
\hline
\end{tabular}




\section{RÍO GRANDE DE AÑASCO BASIN-Continued}

50142710 Río Prieto at Río Prieto, Puerto Rico

LOCATION.--Lat $18^{\circ} 12^{\prime} 06^{\prime \prime}$, long 66 53'05", Hydrologic Unit 21010003, at barrio Río Prieto on Highway 431, 3.7 mi (5.6 km) west from Lago Guayo, and $6.4 \mathrm{mi}(10 \mathrm{~km})$ northeast from Plaza de Maricao.

DRAINAGE AREA.--5.5 $\mathrm{mi}^{2}\left(14 \mathrm{~km}^{2}\right)$. Does not include $9.6 \mathrm{mi}^{2}\left(24.9 \mathrm{~km}^{2}\right)$ in which base flow is diverted to Lago Lucchetti Dam.

LOW-FLOW ESTIMATES.--Based on correlation of 8 base-flow measurements with concurrent base flows at gaging station 50144000 .

REMARKS.--Flow regulated by Lago Prieto Dam.

MAGNITUDE AND FREQUENCY OF ANNUAL LOW FLOWS

\begin{tabular}{cccc}
\hline $\begin{array}{c}\text { Recurrence } \\
\text { interval, } \\
\text { in years }\end{array}$ & \multicolumn{2}{c}{$\begin{array}{c}\text { Lowest average flow, in cubic feet per second, } \\
\text { for indicated number of consecutive days }\end{array}$} \\
\cline { 2 - 4 } & $\mathbf{7}$ & $\mathbf{1 4}$ & $\mathbf{3 0}$ \\
\hline 2 & .9 & 1.1 & 1.2 \\
10 & .5 & .6 & .8 \\
\hline
\end{tabular}

50142900 Río Prieto at Pezuela, Puerto Rico

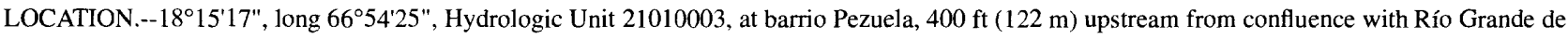
Añasco, and $3.4 \mathrm{mi}(5.5 \mathrm{~km})$ southwest from Plaza de Lares.

DRAINAGE AREA.--16 $\mathrm{mi}^{2}\left(41 \mathrm{~km}^{2}\right)$. Does not include $9.6 \mathrm{mi}^{2}\left(24.9 \mathrm{~km}^{2}\right)$ in which base flow is diverted to Lago Lucchetti Dam.

LOW-FLOW ESTIMATES.--Based on correlation of 7 base-flow measurements with concurrent base flows at gaging station 50144000.

REMARKS.--Flow regulated by Lago Prieto Dam.

MAGNITUDE AND FREQUENCY OF ANNUAL LOW FLOWS

\begin{tabular}{cccc}
\hline $\begin{array}{c}\text { Recurrence } \\
\text { interval, } \\
\text { in years }\end{array}$ & \multicolumn{2}{c}{$\begin{array}{c}\text { Lowest average flow, in cubic feet per second, } \\
\text { for indicated number of consecutive days }\end{array}$} \\
\cline { 2 - 4 } 2 & $\mathbf{7}$ & $\mathbf{1 4}$ & $\mathbf{3 0}$ \\
\hline 10 & 6.6 & 7.4 & 8.1 \\
\hline
\end{tabular}

\section{Río Grande de Añasco near Lares, Puerto Rico}

LOCATION.--Lat $18^{\circ} 15^{\prime} 28^{\prime \prime}$, long 66 55'05", Hydrologic Unit 21010003, at bridge on Highway 124, $0.7 \mathrm{mi}(1.1 \mathrm{~km})$ from confluence of Río Blanco and Río Prieto.

DRAINAGE AREA.--26 $\mathrm{mi}^{2}\left(67 \mathrm{~km}^{2}\right)$. Does not include $36.2 \mathrm{mi}^{2}\left(93.8 \mathrm{~km}^{2}\right)$ in which base flow is diverted to Lago Lucchetti Dam.

LOW-FLOW ESTIMATES.--Based on correlation of 8 base-flow measurements with concurrent base flows at gaging station 50144000.

REMARKS.--Flow regulated by Lago Yahuecas, Lago Guayo and Lago Prieto Dams.

MAGNITUDE AND FREQUENCY OF ANNUAL LOW FLOWS

\begin{tabular}{cccc}
\hline $\begin{array}{c}\text { Recurrence } \\
\text { interval, } \\
\text { in years }\end{array}$ & \multicolumn{2}{c}{$\begin{array}{c}\text { Lowest average flow, in cubic feet per second, } \\
\text { for indicated number of consecutive days }\end{array}$} \\
\cline { 2 - 4 } & $\mathbf{7}$ & $\mathbf{1 4}$ & $\mathbf{3 0}$ \\
\hline 2 & 13 & 15 & 17 \\
10 & 9.0 & 10 & $\mathbf{1 2}$ \\
\hline
\end{tabular}




\section{RÍO GRANDE DE AÑASCO BASIN-Continued}

\section{Río Lajas near Maricao, Puerto Rico}

LOCATION.--Lat 18 10"54", long 66 57'39", Hydrologic Unit 21010003, at barrio Indiera Fría on Highway 105, $0.3 \mathrm{mi}(0.5 \mathrm{~km})$ upstream from confluence with Río Guaba, $0.7 \mathrm{mi}(1.1 \mathrm{~km})$ from Plaza de Maricao.

DRAINAGE AREA.--5.8 $\mathrm{mi}^{2}\left(15 \mathrm{~km}^{2}\right)$.

LOW-FLOW ESTIMATES.--Based on correlation of 8 base-flow measurements with concurrent base flows at gaging station 50144000 .

MAGNITUDE AND FREQUENCY OF ANNUAL LOW FLOWS

\begin{tabular}{cccc}
\hline $\begin{array}{c}\text { Recurrence } \\
\text { interval, }\end{array}$ & \multicolumn{2}{c}{$\begin{array}{c}\text { Lowest average flow, in cubic feet per second, } \\
\text { for indicated number of consecutive days }\end{array}$} \\
\cline { 2 - 4 } in years & $\mathbf{7}$ & $\mathbf{1 4}$ & $\mathbf{3 0}$ \\
\hline 2 & 2.5 & 2.7 & 3.0 \\
10 & 1.7 & 1.8 & 2.2 \\
\hline
\end{tabular}

50143108 Río Guaba near Maricao, Puerto Rico

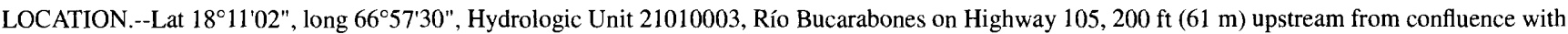
Río Lajas, and $1.5 \mathrm{mi}(2.4 \mathrm{~km})$ from Plaza de Maricao.

DRAINAGE AREA.--5.0 $\mathrm{mi}^{2}\left(13 \mathrm{~km}^{2}\right)$.

LOW-FLOW ESTIMATES.--Based on correlation of 8 base-flow measurements with concurrent base flows at gaging station 50144000.

MAGNITUDE AND FREQUENCY OF ANNUAL LOW FLOWS

\begin{tabular}{cccc}
\hline $\begin{array}{c}\text { Recurrence } \\
\text { interval, } \\
\text { in years }\end{array}$ & \multicolumn{2}{c}{$\begin{array}{c}\text { Lowest average flow, in cubic feet per second, } \\
\text { for indicated number of consecutive days }\end{array}$} \\
\cline { 2 - 4 } & $\mathbf{7}$ & $\mathbf{1 4}$ & $\mathbf{3 0}$ \\
\hline 2 & 3.1 & 3.4 & 3.7 \\
10 & 2.2 & 2.4 & 2.8 \\
\hline
\end{tabular}

50143150 Río Bucarabones near Las Marías, Puerto Rico

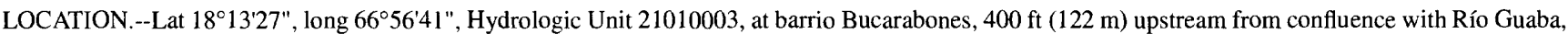
$3.7 \mathrm{mi}(5.6 \mathrm{~km})$ northeast from Plaza de Maricao.

DRAINAGE AREA.--9.2 $\mathrm{mi}^{2}\left(24 \mathrm{~km}^{2}\right)$.

LOW-FLOW ESTIMATES.--Based on correlation of 8 base-flow measurements with concurrent base flows at gaging station 50144000 .

MAGNITUDE AND FREQUENCY OF ANNUAL LOW FLOWS

\begin{tabular}{cccc}
\hline $\begin{array}{c}\text { Recurrence } \\
\text { interval, } \\
\text { in years }\end{array}$ & \multicolumn{2}{c}{$\begin{array}{c}\text { Lowest average flow, in cubic feet per second, } \\
\text { for indicated number of consecutive days }\end{array}$} \\
\cline { 2 - 4 } & $\mathbf{7}$ & $\mathbf{1 4}$ & $\mathbf{3 0}$ \\
\hline 2 & 5.8 & 6.3 & 6.8 \\
10 & 4.2 & 4.5 & 5.2 \\
\hline
\end{tabular}

50143200 Río Guaba near Las Marías, Puerto Rico

LOCATION.--Lat 18 13'37", long 66 56'33", Hydrologic Unit 21010003, at barrio Cerrote on Highway 124, $0.3 \mathrm{mi}(0.5 \mathrm{~km})$ downstream of confluence with Río Bucarabones, and $3.9 \mathrm{mi}(6.3 \mathrm{~km})$ northeast from Plaza de Maricao.

DRAINAGE AREA.--25 $\mathrm{mi}^{2}\left(66 \mathrm{~km}^{2}\right)$.

LOW-FLOW ESTIMATES.--Based on correlation of 8 base-flow measurements with concurrent base flows at gaging station 50144000 .

MAGNITUDE AND FREQUENCY OF ANNUAL LOW FLOWS

\begin{tabular}{cccc}
\hline $\begin{array}{c}\text { Recurrence } \\
\text { interval, } \\
\text { in years }\end{array}$ & \multicolumn{2}{c}{$\begin{array}{c}\text { Lowest average flow, in cubic feet per second, } \\
\text { for indicated number of consecutive days }\end{array}$} \\
\cline { 2 - 4 } 2 & $\mathbf{7}$ & $\mathbf{1 4}$ & $\mathbf{3 0}$ \\
\hline 10 & 12 & 13 & 15 \\
& 8.5 & 9.2 & 11 \\
\hline
\end{tabular}




\section{RÍO GRANDE DE AÑASCO BASIN—Continued}

\section{Quebrada Las Cañas at Perchas, Puerto Rico}

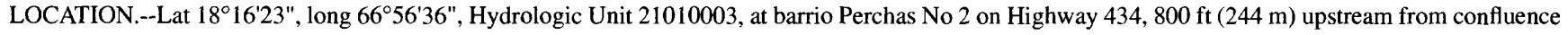
with Río Grande de Añasco, and $3.5 \mathrm{mi}(5.6 \mathrm{~km})$ from Plaza de Las Marías.

DRAINAGE AREA.--3.1 $\mathrm{mi}^{2}\left(8.0 \mathrm{~km}^{2}\right)$.

LOW-FLOW ESTIMATES.--Based on correlation of 8 base-flow measurements with concurrent base flows at gaging station 50144000 . MAGNITUDE AND FREQUENCY OF ANNUAL LOW FLOWS

\begin{tabular}{cccc}
\hline $\begin{array}{c}\text { Recurrence } \\
\text { interval, }\end{array}$ & \multicolumn{2}{c}{$\begin{array}{c}\text { Lowest average flow, in cubic feet per second, } \\
\text { for indicated number of consecutive days }\end{array}$} \\
\cline { 2 - 4 } in years & $\mathbf{7}$ & $\mathbf{1 4}$ & $\mathbf{3 0}$ \\
\hline 2 & 1.7 & 1.9 & 2.1 \\
10 & 1.2 & 1.3 & 1.5 \\
\hline
\end{tabular}

50143500 Río Mayagüecilla at Las Marías, Puerto Rico

LOCATION.--Lat $18^{\circ} 14^{\prime} 50^{\prime \prime}$, long 66 59'05", Hydrologic Unit 21010003, at barrio Palma Escrita on Highway 124, $2.0 \mathrm{mi}(3.2 \mathrm{~km})$ upstream from confluence with Río Grande de Añasco, $0.7 \mathrm{mi}(1.1 \mathrm{~km})$ southeast from Plaza de Las Marías.

DRAINAGE AREA.-- $3.3 \mathrm{mi}^{2}\left(8.5 \mathrm{~km}^{2}\right)$.

LOW-FLOW ESTIMATES.--Based on correlation of 6 base-flow measurements with concurrent low flows at gaging station 50144000 . MAGNITUDE AND FREQUENCY OF ANNUAL LOW FLOWS

\begin{tabular}{cccc}
\hline $\begin{array}{c}\text { Recurrence } \\
\text { interval, } \\
\text { in years }\end{array}$ & \multicolumn{2}{c}{$\begin{array}{c}\text { Lowest average flow, in cubic feet per second, } \\
\text { for indicated number of consecutive days }\end{array}$} \\
\cline { 2 - 3 } 2 & $\mathbf{7}$ & $\mathbf{1 4}$ & $\mathbf{3 0}$ \\
\hline 10 & 1.6 & 1.8 & 2.0 \\
1.1 & 1.2 & 1.4 \\
\hline
\end{tabular}

\section{Río Grande de Añasco near Las Marías, Puerto Rico}

LOCATION.--Lat $18^{\circ} 16^{\prime} 41^{\prime \prime}$, long 66 $58^{\prime} 48^{\prime \prime}$, Hydrologic Unit 21010003 , at barrio Guacio on Highway $119,1.8$ mi (2.9 km) northeast from Plaza de Las Marías.

DRAINAGE AREA.--80 $\mathrm{mi}^{2}\left(207 \mathrm{~km}^{2}\right)$. Does not include $36.2 \mathrm{mi}^{2}\left(93.8 \mathrm{~km}^{2}\right)$ in which base flow is diverted to Lago Lucchetti Dam.

LOW-FLOW ESTIMATES.--Based on correlation of 8 base-flow measurements with concurrent base flows at gaging station 50144000 .

REMARKS.--Flow regulated by Lago Yahuecas, Lago Guayo and Lago Prieto Dams.

MAGNITUDE AND FREQUENCY OF ANNUAL LOW FLOWS

\begin{tabular}{cccc}
\hline $\begin{array}{c}\text { Recurrence } \\
\text { interval, }\end{array}$ & \multicolumn{2}{c}{$\begin{array}{c}\text { Lowest average flow, in cubic feet per second, } \\
\text { for indicated number of consecutive days }\end{array}$} \\
\cline { 2 - 4 } in years & $\mathbf{7}$ & $\mathbf{1 4}$ & $\mathbf{3 0}$ \\
\hline 2 & 51 & 54 & 60 \\
10 & 36 & 40 & 46 \\
\hline
\end{tabular}

50143900 Río Arenas at Las Marías, Puerto Rico

LOCATION.--Lat $18^{\circ} 15^{\prime} 10^{\prime \prime}$, long 66 59'57", Hydrologic Unit 21010003, at barrio Maravillas on Highway 119, 0.5 mi (0.8 km) southwest from Plaza de Las Marías.

DRAINAGE AREA.-- $2.8 \mathrm{mi}^{2}\left(7.2 \mathrm{~km}^{2}\right)$.

LOW-FLOW ESTIMATES.--Based on correlation of 8 base-flow measurements with concurrent base flows at gaging station 50144000 . MAGNITUDE AND FREQUENCY OF ANNUAL LOW FLOWS

\begin{tabular}{cccc}
\hline $\begin{array}{c}\text { Recurrence } \\
\text { interval, }\end{array}$ & \multicolumn{3}{c}{$\begin{array}{c}\text { Lowest average flow, in cubic feet per second, } \\
\text { for indicated number of consecutive days }\end{array}$} \\
\cline { 2 - 4 } & $\mathbf{7}$ & $\mathbf{1 4}$ & $\mathbf{3 0}$ \\
\hline 2 & 2.8 & 3.0 & 3.2 \\
10 & 2.1 & 2.3 & 2.6 \\
\hline
\end{tabular}




\section{RÍO GRANDE DE AÑASCO BASIN-Continued}

\section{Quebrada Cerro Gordo near Cerro Gordo, Puerto Rico}

LOCATION.--Lat $18^{\circ} 17^{\prime} 09^{\prime \prime}$, long 66 $04^{\prime} 09^{\prime \prime}$, Hydrologic Unit 21010003, at barrio Corcovada, $600 \mathrm{ft}$ (183 m) upstream from confluence with Río Grande de Añasco, $5.7 \mathrm{mi}(9.2 \mathrm{~km})$ from Las Marías, and $4.8 \mathrm{mi}(7.7 \mathrm{~km})$ east from Plaza de Añasco.

DRAINAGE AREA.--2.7 $\mathrm{mi}^{2}\left(6.9 \mathrm{~km}^{2}\right)$.

LOW-FLOW ESTIMATES.--Based on correlation of 8 base-flow measurements with concurrent base flows at gaging station 50144000.

MAGNITUDE AND FREQUENCY OF ANNUAL LOW FLOWS

\begin{tabular}{cccc}
\hline $\begin{array}{c}\text { Recurrence } \\
\text { interval, }\end{array}$ & \multicolumn{2}{c}{$\begin{array}{c}\text { Lowest average flow, in cubic feet per second, } \\
\text { for indicated number of consecutive days }\end{array}$} \\
\cline { 2 - 4 } in years & $\mathbf{7}$ & $\mathbf{1 4}$ & $\mathbf{3 0}$ \\
\hline 2 & 1.7 & 1.8 & 2.0 \\
10 & 1.2 & 1.3 & 1.5 \\
\hline
\end{tabular}

50144900 Río Humata near El Espino, Puerto Rico

LOCATION.--Lat $18^{\circ} 17^{\prime} 18^{\prime \prime}$, long 6706'24", Hydrologic Unit 21010003, at barrio Carreras on 109, $0.3 \mathrm{mi}(0.5 \mathrm{~km})$ upstream from confluence with Río Grande de Añasco, and $2.4 \mathrm{mi}(3.9 \mathrm{~km})$ east from Plaza de Añasco.

DRAINAGE AREA.--4.9 $\mathrm{mi}^{2}\left(13 \mathrm{~km}^{2}\right)$.

LOW-FLOW ESTIMATES.--Based on correlation of 8 base-flow measurements with concurrent base flows at gaging station 50144000 .

MAGNITUDE AND FREQUENCY OF ANNUAL LOW FLOWS

\begin{tabular}{cccc}
\hline $\begin{array}{c}\text { Recurrence } \\
\text { interval, } \\
\text { in years }\end{array}$ & \multicolumn{2}{c}{$\begin{array}{c}\text { Lowest average flow, in cubic feet per second, } \\
\text { for indicated number of consecutive days }\end{array}$} \\
\cline { 2 - 4 } 2 & $\mathbf{7}$ & $\mathbf{1 4}$ & $\mathbf{3 0}$ \\
\hline 10 & 1.9 & 2.1 & 2.4 \\
& 1.3 & 1.4 & 1.7 \\
\hline
\end{tabular}

50145000 Río Grande de Añasco at El Espino, Puerto Rico

LOCATION.--Lat $18^{\circ} 16^{\prime} 50^{\prime \prime}$, long 67 $06^{\prime} 46^{\prime \prime}$, Hydrologic Unit 21010003, at barrio Espino on Highway $406,400 \mathrm{ft}(249 \mathrm{~m})$ east from intersection with Highway 109, and $1.9 \mathrm{mi}(3.1 \mathrm{~km})$ from Plaza de Añasco.

DRAINAGE AREA.--108 $\mathrm{mi}^{2}\left(280 \mathrm{~km}^{2}\right)$. Does not include $36.2 \mathrm{mi}^{2}\left(103 \mathrm{~km}^{2}\right)$ in which base flow is diverted to Lago Lucchetti Dam.

LOW-FLOW ESTIMATES.--Based on correlation of 8 base-flow measurements with concurrent base flows at gaging station 50144000.

REMARKS.--Flow regulated by Lago Yahuecas, Lago Guayo and Lago Prieto Dams.

MAGNITUDE AND FREQUENCY OF ANNUAL LOW FLOWS

\begin{tabular}{cccc}
\hline $\begin{array}{c}\text { Recurrence } \\
\text { interval, }\end{array}$ & \multicolumn{2}{c}{$\begin{array}{c}\text { Lowest average flow, in cubic feet per second, } \\
\text { for indicated number of consecutive days }\end{array}$} \\
\cline { 2 - 4 } in years & $\mathbf{7}$ & $\mathbf{1 4}$ & $\mathbf{3 0}$ \\
\hline 2 & 68 & 74 & 83 \\
10 & 49 & 54 & 62 \\
\hline
\end{tabular}

50145400 Río Casei near Mayagüez, Puerto Rico

LOCATION.--Lat $18^{\circ} 15^{\prime} 18^{\prime \prime}$, long 6704'48", Hydrologic Unit 21010003, at barrio Legüísamo on Highway 108, 4.6 mi (7.4 km) northeast from Mayagüez, and $4.5 \mathrm{mi}(7.2 \mathrm{~km})$ southeast from Plaza de Mayagüez.

DRAINAGE AREA.--8.2 $\mathrm{mi}^{2}\left(21 \mathrm{~km}^{2}\right)$.

LOW-FLOW ESTIMATES.--Based on correlation of 8 base-flow measurements with concurrent base flows at gaging station 50136000 .

MAGNITUDE AND FREQUENCY OF ANNUAL LOW FLOWS

\begin{tabular}{cccc}
\hline $\begin{array}{c}\text { Recurrence } \\
\text { interval, }\end{array}$ & \multicolumn{2}{c}{$\begin{array}{c}\text { Lowest average flow, in cubic feet per second, } \\
\text { for indicated number of consecutive days }\end{array}$} \\
\cline { 2 - 4 } in years & $\mathbf{7}$ & $\mathbf{1 4}$ & $\mathbf{3 0}$ \\
\hline & & 6.5 & 6.8 \\
10 & 6.0 & 3.6 & 4.2 \\
\hline
\end{tabular}




\section{RÍO GRANDE DE AÑASCO BASIN—Continued}

\section{Río Grande de Añasco at Añasco Arriba, Puerto Rico}

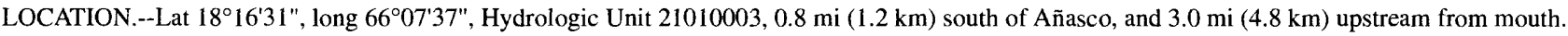
DRAINAGE AREA.--139 $\mathrm{mi}^{2}\left(360 \mathrm{~km}^{2}\right)$. Does not include $36.2 \mathrm{mi}^{2}\left(103 \mathrm{~km}^{2}\right)$ in which base flow is diverted to Lago Lucchetti Dam.

LOW-FLOW ESTIMATES.--Based on correlation of 8 base-flow measurements with concurrent base flows at gaging station 50144000 .

REMARKS.--Flow regulated by Lago Yahuecas, Lago Guayo and Lago Prieto Dams.

MAGNITUDE AND FREQUENCY OF ANNUAL LOW FLOWS

\begin{tabular}{cccc}
\hline $\begin{array}{c}\text { Recurrence } \\
\text { interval, }\end{array}$ & \multicolumn{2}{c}{$\begin{array}{c}\text { Lowest average flow, in cubic feet per second, } \\
\text { for indicated number of consecutive days }\end{array}$} \\
\cline { 2 - 4 } in years & $\mathbf{7}$ & $\mathbf{1 4}$ & $\mathbf{3 0}$ \\
\hline 2 & 73 & 80 & 89 \\
10 & 50 & 55 & 66 \\
\hline
\end{tabular}

50146002 Río Cañas at Río Cañas Arriba, Puerto Rico

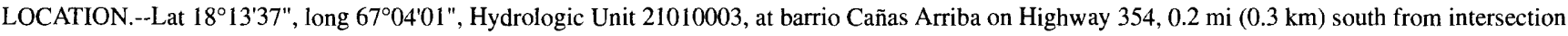
with Highway 355, and $5.1 \mathrm{mi}(8.2 \mathrm{~km})$ from Plaza de Mayagüez.

DRAINAGE AREA.--3.6 $\mathrm{mi}^{2}\left(9.3 \mathrm{~km}^{2}\right)$

LOW-FLOW ESTIMATES.--Based on correlation of 8 base-flow measurements with concurrent base flows at gaging station 50136000 .

MAGNITUDE AND FREQUENCY OF ANNUAL LOW FLOWS

\begin{tabular}{cccc}
\hline $\begin{array}{c}\text { Recurrence } \\
\text { interval, }\end{array}$ & \multicolumn{2}{c}{$\begin{array}{c}\text { Lowest average flow, in cubic feet per second, } \\
\text { for indicated number of consecutive days }\end{array}$} \\
\cline { 2 - 4 } in years & $\mathbf{7}$ & $\mathbf{1 4}$ & $\mathbf{3 0}$ \\
\hline 2 & 2.2 & 2.4 & 2.5 \\
10 & 1.2 & 1.3 & 1.4 \\
\hline
\end{tabular}

50146005 Río Cañas at Río Cañas Abajo, Puerto Rico

LOCATION.--Lat $18^{\circ} 14^{\prime} 38^{\prime \prime}$, long $67^{\circ} 07^{\prime} 17^{\prime \prime}$, Hydrologic Unit 21010003, at barrio Río Cañas Abajo on Highway 108 , and $3.1 \mathrm{mi}$ (5.0 km) northeast from Plaza de Mayagüez.

DRAINAGE AREA.-- $11 \mathrm{mi}^{2}\left(29 \mathrm{~km}^{2}\right)$.

LOW-FLOW ESTIMATES.--Based on correlation of 8 base-flow measurements with concurrent base flows at gaging station 50136000. MAGNITUDE AND FREQUENCY OF ANNUAL LOW FLOWS

\begin{tabular}{cccc}
\hline $\begin{array}{c}\text { Recurrence } \\
\text { interval, }\end{array}$ & \multicolumn{2}{c}{$\begin{array}{c}\text { Lowest average flow, in cubic feet per second, } \\
\text { for indicated number of consecutive days }\end{array}$} \\
\cline { 2 - 4 } in years & $\mathbf{7}$ & $\mathbf{1 4}$ & $\mathbf{3 0}$ \\
\hline 2 & 2.6 & 3.0 & 3.2 \\
10 & 1.0 & 1.2 & 1.4 \\
\hline
\end{tabular}

50146075 Río Dagüey near Añasco, Puerto Rico

LOCATION.--Lat $18^{\circ} 17^{\prime} 19^{\prime \prime}$, long $67^{\circ} 08^{\prime} 08^{\prime \prime}$, Hydrologic Unit 21010003 , at barrio Carreras on Highway $405,100 \mathrm{ft}$ (30 m) east from intersection with Highway 404, and $0.5 \mathrm{mi}(0.8 \mathrm{~km})$ northeast from Plaza de Mayagüez.

DRAINAGE AREA.-- $1.1 \mathrm{mi}^{2}\left(2.8 \mathrm{~km}^{2}\right)$.

LOW-FLOW ESTIMATES.--Based on correlation of 8 base-flow measurements with concurrent base flows at gaging station 50136000 . MAGNITUDE AND FREQUENCY OF ANNUAL LOW FLOWS

\begin{tabular}{cccc}
\hline $\begin{array}{c}\text { Recurrence } \\
\text { interval, } \\
\text { in years }\end{array}$ & $\begin{array}{c}\text { Lowest average flow, in cubic feet per second, } \\
\text { for indicated number of consecutive days }\end{array}$ \\
\cline { 2 - 4 } 2 & $\mathbf{7}$ & $\mathbf{1 4}$ & $\mathbf{3 0}$ \\
\hline 10 & .3 & .3 & .3 \\
.1 & .1 & .1 \\
\hline
\end{tabular}




\section{RÍO GRANDE BASIN}

\section{Río Grande near Rincón, Puerto Rico}

LOCATION.--Lat $18^{\circ} 22^{\prime} 06^{\prime \prime}$, long 67 $13^{\prime} 56^{\prime \prime}$, Hydrologic Unit 21010003, at bridge on Highway 115, $1.2 \mathrm{mi}(1.9 \mathrm{~km})$ upstream from mouth, and $2.2 \mathrm{mi}(3.5$ km) northeast of Rincón.

DRAINAGE AREA.-- $2.8 \mathrm{mi}^{2}\left(7.3 \mathrm{~km}^{2}\right)$.

LOW-FLOW ESTIMATES.--Based on correlation of 8 base-flow measurements with concurrent base flows at gaging station 50144000 .

MAGNITUDE AND FREQUENCY OF ANNUAL LOW FLOWS

\begin{tabular}{cccc}
\hline $\begin{array}{c}\text { Recurrence } \\
\text { interval, } \\
\text { in years }\end{array}$ & \multicolumn{2}{c}{$\begin{array}{c}\text { Lowest average flow, in cubic feet per second, } \\
\text { for indicated number of consecutive days }\end{array}$} \\
\cline { 2 - 4 } 2 & $\mathbf{7}$ & $\mathbf{1 4}$ & $\mathbf{3 0}$ \\
\hline 10 & .3 & .3 & .4 \\
\hline & .2 & .2 & .2 \\
\hline
\end{tabular}

50146300 Río Ingenio at Jagüey, Puerto Rico

LOCATION.--Lat $18^{\circ} 20^{\prime} 36^{\prime \prime}$, long $67^{\circ} 11^{\prime} 52^{\prime \prime}$, Hydrologic Unit 21010003 , at barrio Jagüey at unnumbered highway, $0.3 \mathrm{mi}(0.5 \mathrm{~km})$ from intersection of Highway 411 , and $2.7 \mathrm{mi}(4.3 \mathrm{~km})$ southwest from Plaza de Aguada.

DRAINAGE AREA.-- $3.2 \mathrm{mi}^{2}\left(8.2 \mathrm{~km}^{2}\right)$.

LOW-FLOW ESTIMATES.--Based on correlation of 8 base-flow measurements with concurrent base flows at gaging station 50144000 .

MAGNITUDE AND FREQUENCY OF ANNUAL LOW FLOWS

\begin{tabular}{cccc}
\hline $\begin{array}{c}\text { Recurrence } \\
\text { interval, }\end{array}$ & \multicolumn{2}{c}{$\begin{array}{c}\text { Lowest average flow, in cubic feet per second, } \\
\text { for indicated number of consecutive days }\end{array}$} \\
\cline { 2 - 4 } in years & $\mathbf{7}$ & $\mathbf{1 4}$ & $\mathbf{3 0}$ \\
\hline 2 & 1.1 & 1.2 & 1.4 \\
10 & .7 & .8 & 1.0 \\
\hline
\end{tabular}

50146400 Río Ingenio near Aguada, Puerto Rico

LOCATION.--Lat $18^{\circ} 22^{\prime} 48^{\prime \prime}$, long $67^{\circ} 12^{\prime} 35^{\prime \prime}$, Hydrologic Unit 21010003 , at bridge on unimproved road, $0.3 \mathrm{mi}(0.5 \mathrm{~km})$ upstream from confluence with Río Culebra, and $1.4 \mathrm{mi}(2.3 \mathrm{~km})$ west of Aguada.

DRAINAGE AREA. $-7.0 \mathrm{mi}^{2}\left(18 \mathrm{~km}^{2}\right)$.

LOW-FLOW ESTIMATES.--Based on correlation of 8 base-flow measurements with concurrent base flows at gaging station 50144000 .

MAGNITUDE AND FREQUENCY OF ANNUAL LOW FLOWS

\begin{tabular}{|c|c|c|c|}
\hline \multirow{2}{*}{$\begin{array}{l}\text { Recurrence } \\
\text { interval, } \\
\text { in years }\end{array}$} & \multicolumn{3}{|c|}{$\begin{array}{l}\text { Lowest average flow, in cubic feet per second, } \\
\text { for indicated number of consecutive days }\end{array}$} \\
\hline & 7 & 14 & 30 \\
\hline $\begin{array}{r}2 \\
10\end{array}$ & $\begin{array}{l}1.6 \\
1.0\end{array}$ & $\begin{array}{l}1.9 \\
1.1\end{array}$ & $\begin{array}{l}2.1 \\
1.4\end{array}$ \\
\hline
\end{tabular}




\section{RÍO CULEBRA BASIN}

\section{Río Culebra near Aguada, Puerto Rico}

LOCATION.--Lat $18^{\circ} 22^{\prime} 26^{\prime \prime}$, long 67 $11^{\prime} 35^{\prime \prime}$, Hydrologic Unit 21010003, at bridge on Highway 411, $0.6 \mathrm{mi}(1.0 \mathrm{~km})$ south of Aguada, $1.5 \mathrm{mi}(2.4 \mathrm{~km})$ upstream from confluence with Río Ingenio, and $1.9 \mathrm{mi}(3.1 \mathrm{~km})$ upstream from mouth of Río Guayabo.

DRAINAGE AREA.--3.8 $\mathrm{mi}^{2}\left(9.7 \mathrm{~km}^{2}\right)$.

LOW-FLOW ESTIMATES.--Based on correlation of 8 base-flow measurements with concurrent base flows at gaging station 50144000 .

REMARKS.--Minor diversions are made above the station to filter plant for public-water supply.

MAGNITUDE AND FREQUENCY OF ANNUAL LOW FLOWS

\begin{tabular}{cccc}
\hline $\begin{array}{c}\text { Recurrence } \\
\text { interval, } \\
\text { in years }\end{array}$ & \multicolumn{2}{c}{$\begin{array}{c}\text { Lowest average flow, in cubic feet per second, } \\
\text { for indicated number of consecutive days }\end{array}$} \\
\cline { 2 - 4 } & $\mathbf{7}$ & $\mathbf{1 4}$ & $\mathbf{3 0}$ \\
\hline 2 & .8 & 1.0 & 1.1 \\
10 & .5 & .6 & .7 \\
\hline
\end{tabular}

